Supporting Information for

\title{
1,2-Azaborine's Distinct Electronic Structure Unlocks Two New Regioisomeric Building Blocks via Resolution Chemistry
}

Cameron R. McConnell, Fredrik Haeffner, Andrew W. Baggett, and Shih-Yuan Liu*

\section{Contents}

1. General

2. Syntheses

2.1 Borylation of $\mathbf{1}$

2.2 Preparatory Scale Separations of 5Bpin and 4Bpin

2.3 Electrophilic 1,2-Azaborine Building Blocks

3. Kinetic and Thermodynamic Experiments

3.1 Oxidation Kinetics

3.2 Protodeborylation Kinetics

3.3 Transesterification Kinetics and Thermodynamics

4. Computational Details

4.1 Ground State Calculations

4.2 Oxidation Calculations

4.3 Transesterification Calculations

S36-S43

5. NMR Spectra

S44-S59

6. References 


\section{General}

All oxygen- and moisture-sensitive manipulations were carried out under an inert atmosphere using either standard Schlenk techniques or a glove box.

THF, $\mathrm{Et}_{2} \mathrm{O}, \mathrm{CH}_{2} \mathrm{Cl}_{2}$ and pentane were purified by passing through a neutral alumina column under argon. Methanol, $\mathrm{CD}_{2} \mathrm{Cl}_{2}$, and $\mathrm{C}_{6} \mathrm{D}_{6}$ were purified by distillation after drying over calcium hydride. THF- $\mathrm{D}_{8}$ was purified by distillation after drying over sodium/benzophenone.

All other chemicals and solvents were purchased (TCI, Aldrich, Acros, AK Scientific, or Strem) and used as received.

${ }^{1} \mathrm{H}$ NMR spectra were recorded on a Varian Inova 600 spectrometer or Varian VNMRS 500 spectrometer. ${ }^{13} \mathrm{C}$ NMR spectra were recorded on a Varian Inova 600 spectrometer or Varian VNMRS 500 spectrometer. ${ }^{1} \mathrm{H}$ and ${ }^{13} \mathrm{C}$ were referenced internally to solvent signals $\left(\mathrm{CD}_{2} \mathrm{Cl}_{2}\right.$ : $5.32 \mathrm{ppm}$ for ${ }^{1} \mathrm{H} \mathrm{NMR}, 53.84 \mathrm{ppm}$ for ${ }^{13} \mathrm{C}$ NMR; $\mathrm{C}_{6} \mathrm{D}_{6}: 7.16 \mathrm{ppm}$ for ${ }^{1} \mathrm{H}$ NMR, $128.06 \mathrm{ppm}$ for ${ }^{13} \mathrm{C}$ NMR; THF-D $8.58 \mathrm{ppm}$ for ${ }^{1} \mathrm{H}$ NMR, $67.21 \mathrm{ppm}$ for ${ }^{13} \mathrm{C}$ NMR). ${ }^{11} \mathrm{~B}$ NMR spectra were recorded on a Varian Inova 600 spectrometer or Varian Inova 500 spectrometer at ambient temperature. ${ }^{11} \mathrm{~B}$ NMR shifts were externally referenced to $\mathrm{BF}_{3} \bullet \mathrm{Et}_{2} \mathrm{O}(\delta 0)$. High-resolution mass spectrometry data were obtained at the Boston College mass spectrometry facility on a JEOL AccuTOF instrument (JEOL USA, Peabody, MA), equipped with a DART ion source (IonSense, Inc., Danvers, MA) in positive ion mode. IR spectra were recorded on a Bruker Alpha FT-IR instrument with OPUS software.

\section{Syntheses}

\subsection{Borylation of Compound N-TBS-B-Mes-1,2-Azaborine 1}

Compound 1 was synthesized as a white solid according to a published procedure. Spectra of the isolated compound matched published values. ${ }^{1}$

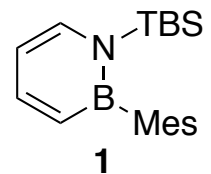

Mixture of Compounds 5Bpin and 4Bpin. Inside a glove box, a $150 \mathrm{~mL}$ pressure vessel was charged with $[\operatorname{Ir}(\mathrm{OMe})(\mathrm{cod})]_{2}(72 \mathrm{mg}, 0.11 \mathrm{mmol}), 4,4$ '-di-tert-butyl-2,2'bipyridine (dtbpy) $(58 \mathrm{mg}, 0.22 \mathrm{mmol})$, bis(pinacolato)diboron $\left(\mathrm{B}_{2} \operatorname{pin}_{2}\right)(2.00 \mathrm{~g}, 7.95$ mmol), and MTBE $(35.0 \mathrm{~mL})$. The catalyst mixture was aged for 2 minutes. Neat 1 $(2.25 \mathrm{~g}, 7.23 \mathrm{mmol})$ was added to the catalyst mixture and the reaction vessel was sealed. The reaction mixture was transferred to a fume hood heated at $80{ }^{\circ} \mathrm{C}$ for 1 hour, at which point the reaction was determined to be complete by ${ }^{1} \mathrm{H}$ NMR. After cooling, volatiles were removed under reduced pressure and the crude residue was purified by silica gel chromatography with 99:1 pentane:ether as the eluent. A 1.2:1 C5:C4 mixture of borylated isomers was obtained as a white solid in $92 \%$ yield. The ratio was determined by ${ }^{1} \mathrm{H}$ NMR integration against a 1,3,5-trimethoxybenzene internal standard. 


\subsection{Preparatory Scale Separations of Borylated Isomers}

Compound 5Bpin (via NMO oxidation). Inside a glovebox, a $250 \mathrm{~mL}$ round bottom flask was charged with a 1.2:1 mixture of 5Bpin (5.31 g, $12.1 \mathrm{mmol})$ and 4Bpin (4.35 g, 9.9 mmol), NMO (5.69 g, $48.6 \mathrm{mmol})$, and $\mathrm{CH}_{2} \mathrm{Cl}_{2}(80.0 \mathrm{~mL})$.

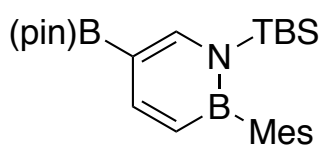
The reaction mixture was allowed to stir at room temperature for 24 hours. Reaction progress was monitored by ${ }^{1} \mathrm{H}$ NMR. The reaction mixture was removed from the glovebox and saturated $\mathrm{NaHCO}_{3}$ solution was added and the crude product was extracted with excess dichloromethane. The organic layer was dried over $\mathrm{Na}_{2} \mathrm{SO}_{4}$, passed through a Buchner funnel lined with filter paper, and concentrated under reduced pressure. Pure 5Bpin was recovered as a white solid after purification by silica gel chromatography with 95:5 pentane:ether as the eluent $(5.14 \mathrm{~g}, 11.8 \mathrm{mmol}$, $97 \%$ based on $12.1 \mathrm{mmol}$ starting material). ${ }^{1} \mathrm{H} \mathrm{NMR}\left(500 \mathrm{MHz}, \mathrm{CD}_{2} \mathrm{Cl}_{2}\right) \delta 8.04(\mathrm{~s}$, $1 \mathrm{H}), 7.81(\mathrm{dd}, \mathrm{J}=10.8,1.3 \mathrm{~Hz}, 1 \mathrm{H}), 6.78(\mathrm{~s}, 2 \mathrm{H}), 6.54(\mathrm{~m}, 1 \mathrm{H}), 2.28(\mathrm{~s}, 3 \mathrm{H}), 2.06$ (s, $6 \mathrm{H}), 1.32(\mathrm{~s}, 12 \mathrm{H}), 0.92(\mathrm{~s}, 9 \mathrm{H}), 0.03(\mathrm{~s}, 6 \mathrm{H}) \cdot 30.71 .{ }^{13} \mathrm{C} \mathrm{NMR}\left(126 \mathrm{MHz}, \mathrm{CD}_{2} \mathrm{Cl}_{2}\right) \delta$ $148.11,146.43,138.47,136.29,126.73,83.17,27.05,24.58,23.02,20.81,18.97$, 3.63. (the $\mathrm{C} 3, \mathrm{C} 5$, and mesityl carbons adjacent to boron were not observed). ${ }^{11} \mathrm{~B}$ NMR (160 MHz, $\left.\mathrm{CD}_{2} \mathrm{Cl}_{2}\right) \delta 40.45,30.71$. FTIR (thin film): $\tilde{v}=2976,2931,2860$, 1604. HRMS (DART) calcd. for $\mathrm{C}_{25} \mathrm{H}_{42} \mathrm{~B}_{2} \mathrm{NO}_{2} \mathrm{Si}\left([\mathrm{M}+\mathrm{H}]^{+}\right)$438.31654, found 438.31624 .

Compound 4OH (via NMO oxidation). From the same mixture of products, pure $\mathbf{4 O H}$ was obtained as a yellow oil after purification by silica gel chromatography with $4: 1$ pentane:ether as the eluent $(2.1 \mathrm{~g}, 6.4 \mathrm{mmol}, 65 \%$ yield based

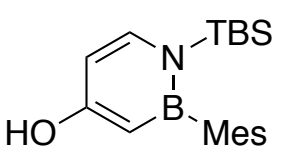
on $9.9 \mathrm{mmol}$ starting material). ${ }^{1} \mathrm{H} \mathrm{NMR}\left(500 \mathrm{MHz}, \mathrm{CD}_{2} \mathrm{Cl}_{2}\right): \delta 7.47(\mathrm{~d}, \mathrm{~J}=7.5 \mathrm{~Hz}$, $1 \mathrm{H}), 6.82(\mathrm{~s}, 2 \mathrm{H}), 6.10(\mathrm{~m}, 2 \mathrm{H}), 5.49(\mathrm{~d}, \mathrm{~J}=3.0 \mathrm{~Hz}, 1 \mathrm{H}), 2.32(\mathrm{~s}, 3 \mathrm{H}), 2.15(\mathrm{~s}, 6 \mathrm{H})$, $0.97(\mathrm{~s}, 9 \mathrm{H}), 0.04(\mathrm{~s}, 6 \mathrm{H}) .{ }^{13} \mathrm{C}$ NMR $\left(126 \mathrm{MHz}, \mathrm{CD}_{2} \mathrm{Cl}_{2}\right): \delta 166.3,142.7,139.4$, $136.8,127.4,105.5,27.9,23.8,21.6,19.8,-2.9$ (C-3 and mesityl C-signal not observed). ${ }^{11} \mathrm{~B}$ NMR (160 MHz, $\left.\mathrm{CD}_{2} \mathrm{Cl}_{2}\right): \delta$ 40.9. FTIR (thin film): $\tilde{v}=3390,2959$, 2929, 2857, 1613. HRMS (DART) calcd. for $\mathrm{C}_{19} \mathrm{H}_{31} \mathrm{BNOSi}[\mathrm{M}+\mathrm{H}]^{+}$328.22680, found 328.22734 .

Compound 4BPin (via protodeborylation). Inside a glovebox, a $150 \mathrm{~mL}$ pressure vessel was charged with a 1:1.4 mixture of 4Bpin (2.06 g, $4.71 \mathrm{mmol})$ and 5Bpin compounds $(2.84 \mathrm{~g}$, $6.49 \mathrm{mmol})$, methanol (1.51 g, $47 \mathrm{mmol})$, [ $\operatorname{Ir}(\mathrm{COD}) \mathrm{OMe}]_{2}(78$

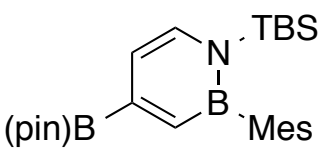
$\mathrm{mg}, 0.12 \mathrm{mmol})$, and $\mathrm{CH}_{2} \mathrm{Cl}_{2}(30.0 \mathrm{~mL})$. The reaction vessel was sealed and transferred to a fume hood. The reaction mixture was heated at $45{ }^{\circ} \mathrm{C}$ for 5 hours. Reaction progress was monitored by ${ }^{1} \mathrm{H}$ NMR. Volatiles were removed under reduced pressure. The product was obtained as a white solid after purification by silica gel chromatography with hexanes as the eluent $(1.22 \mathrm{~g}, 2.78 \mathrm{mmol} 60 \%$ based on 4.71 mmol starting material). (2.25 g, $7.21 \mathrm{mmol}, 111 \%$ of Compound 1 was also recovered based on $6.49 \mathrm{mmol}$ of 5Bpin starting material). ${ }^{1} \mathrm{H}$ NMR (500 MHz, $\left.\mathrm{CD}_{2} \mathrm{Cl}_{2}\right) \delta 7.45(\mathrm{dd}, \mathrm{J}=6.7,1.5 \mathrm{~Hz}, 1 \mathrm{H}), 7.14(\mathrm{~s}, 1 \mathrm{H}), 6.78(\mathrm{~s}, 2 \mathrm{H}), 6.66(\mathrm{dt}, \mathrm{J}=6.9$, $1.7 \mathrm{~Hz}, 1 \mathrm{H}), 2.28(\mathrm{~s}, 3 \mathrm{H}), 2.06(\mathrm{~s}, 6 \mathrm{H}), 1.32(\mathrm{~s}, 12 \mathrm{H}), 0.92(\mathrm{~s}, 9 \mathrm{H}), 0.02(\mathrm{~s}, 6 \mathrm{H}) .{ }^{13} \mathrm{C}$ NMR $\left(126 \mathrm{MHz}, \mathrm{CD}_{2} \mathrm{Cl}_{2}\right) \delta 138.52,136.94,136.09,126.66,114.43,83.75,27.14$, 24.65, 23.18, 20.81, 18.99, -3.64. (the C3, C4, and mesityl carbons adjacent to boron 
were not observed). ${ }^{11} \mathrm{~B}$ NMR (160 MHz, $\left.\mathrm{CD}_{2} \mathrm{Cl}_{2}\right) \delta 39.62,30.35$. FTIR (thin film): $\tilde{v}$ $=2978,2932,2860,1610$. HRMS (DART) calcd. for $\mathrm{C}_{25} \mathrm{H}_{42} \mathrm{~B}_{2} \mathrm{NO}_{2} \mathrm{Si}\left([\mathrm{M}+\mathrm{H}]^{+}\right)$ 438.3171 , found 438.3184 .

Compound 4BO2N. Inside a glovebox, a $100 \mathrm{~mL}$ round bottom flask was charged with a 1:1 mixture of 4Bpin and 5Bpin (1.50 g, $3.46 \mathrm{mmol})$, diethanolamine (0.144 g, 1.37 mmol, 0.4 equiv), and MTBE $(15.0 \mathrm{~mL})$. The reaction

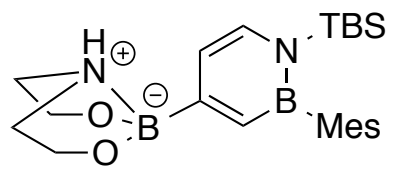
mixture was allowed to stir at room temperature for 4 hours. The formation of a precipitate was observed. Then, the reaction mixture was diluted with pentane and passed through a medium-grained fritted funnel. The collected precipitate was washed copiously with diethyl ether and then dried under reduced pressure. The desired product was obtained as a white powder $(0.370 \mathrm{~g}, 0.87 \mathrm{mmol}, 51 \%$, based on 1.72 mmol starting material). ${ }^{1} \mathrm{H}$ NMR $\left(500 \mathrm{MHz}, \mathrm{CD}_{2} \mathrm{Cl}_{2}\right) \delta 7.39(\mathrm{~d}, \mathrm{~J}=6.8 \mathrm{~Hz}, 1 \mathrm{H}), 6.79$ $(\mathrm{s}, 2 \mathrm{H}), 6.66(\mathrm{~s}, 1 \mathrm{H}), 6.61(\mathrm{~d}, \mathrm{~J}=6.7 \mathrm{~Hz}, 1 \mathrm{H}), 4.45(\mathrm{~s}, 1 \mathrm{H}), 4.09(\mathrm{~m}, 2 \mathrm{H}), 3.93(\mathrm{~d}, \mathrm{~J}=$ $5.3 \mathrm{~Hz}, 2 \mathrm{H}), 3.26(\mathrm{~m}, 2 \mathrm{H}), 2.85(\mathrm{~s}, 2 \mathrm{H}), 2.30(\mathrm{~s}, 3 \mathrm{H}), 2.10(\mathrm{~s}, 6 \mathrm{H}), 0.95(\mathrm{~s}, 9 \mathrm{H}), 0.02$ (s, 6H). ${ }^{13} \mathrm{C}$ NMR $\left(126 \mathrm{MHz}, \mathrm{CD}_{2} \mathrm{Cl}_{2}\right) \delta 138.67,136.44,135.74,126.58,114.99$, $63.16,51.80,27.23,26.69,23.31,20.79,-3.59$. (the carbons adjacent to boron were not observed). ${ }^{11} \mathrm{~B}$ NMR $\left(160 \mathrm{MHz}, \mathrm{CD}_{2} \mathrm{Cl}_{2}\right) \delta 39.06,11.09$. FTIR (thin film): $\tilde{v}=$ 3084, 2959, 2929, 2857, 1606. HRMS (DART) calcd. for $\mathrm{C}_{23} \mathrm{H}_{38} \mathrm{~B}_{2} \mathrm{~N}_{2} \mathrm{O}_{2} \mathrm{Si}\left([\mathrm{M}+\mathrm{H}]^{+}\right)$ 425.2967, found 425.2987 .

Compound 5BO2N. Inside a glovebox, a $20 \mathrm{~mL}$ round bottom flask was charged with 5Bpin (400 mg, 0.91 mmol), diethanolamine (77 $\mathrm{mg}, 0.73 \mathrm{mmol}$ ), and MTBE $(5.0 \mathrm{~mL})$. The reaction mixture was allowed to stir at room temperature for 4 hours. The formation of a precipitate was observed. Then, the reaction mixture was

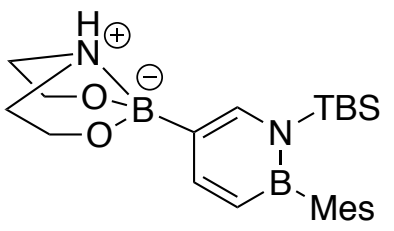
diluted with pentane and passed through a medium-grained fritted funnel. The collected precipitate was washed copiously with diethyl ether and then dried under reduced pressure. The desired product (1:1 mixture with diethanolamine) was obtained as a white solid (170 mg, 33\%). ${ }^{1} \mathrm{H}$ NMR (500 MHz, THF-D 8$) \delta 7.74(\mathrm{~d}, J=$ $4.8 \mathrm{~Hz}, 1 \mathrm{H}), 7.71(\mathrm{~d}, J=1.3 \mathrm{~Hz}, 1 \mathrm{H}), 6.69(\mathrm{~s}, 2 \mathrm{H}), 6.37(\mathrm{~d}, J=10.7 \mathrm{~Hz}, 1 \mathrm{H}), 6.08(\mathrm{~s}$, $1 \mathrm{H}), 3.97(\mathrm{~s}, 2 \mathrm{H}), 3.86(\mathrm{~s}, 2 \mathrm{H}), 3.14(\mathrm{~s}, 2 \mathrm{H}), 2.81(\mathrm{~s}, 2 \mathrm{H}), 2.22(\mathrm{~s}, 3 \mathrm{H}), 2.04(\mathrm{~s}, 6 \mathrm{H})$, $0.94(\mathrm{~s}, 9 \mathrm{H}),-0.03(\mathrm{~s}, 6 \mathrm{H}) .{ }^{13} \mathrm{C}$ NMR $\left(126 \mathrm{MHz}, \mathrm{THF}-\mathrm{D}_{8}\right) \delta 150.54,143.89,139.98$, $136.96,128.25,65.26,52.86,29.03,24.75,22.14,20.72$, -1.86. (the carbons adjacent to boron were not observed). ${ }^{11} \mathrm{~B}$ NMR $\left(160 \mathrm{MHz}, \mathrm{CD}_{2} \mathrm{Cl}_{2}\right) \delta 36.67,8.81$. FTIR (thin film): $\tilde{v}=3297,3085,2930,2860,1599$. HRMS (DART) calcd. for $\mathrm{C}_{23} \mathrm{H}_{38} \mathrm{~B}_{2} \mathrm{~N}_{2} \mathrm{O}_{2} \mathrm{Si}$ $\left([\mathrm{M}+\mathrm{H}]^{+}\right)$425.29614, found 425.29588. 


\subsection{Electrophilic 1,2-Azaborine Building Blocks and their Cross-Coupling Reactions}

Compound 4OTs. Inside a glovebox, a $50 \mathrm{~mL}$ round bottom flask was charged with $\mathbf{4 O H}(2.00 \mathrm{~g}, 6.11 \mathrm{mmol})$, triethylamine $(3.09 \mathrm{~g}, 30.55 \mathrm{mmol}, 4.26 \mathrm{~mL})$, triethylamine hydrogenchloride $(10 \mathrm{mg})$, and $\mathrm{CH}_{2} \mathrm{Cl}_{2}(10.0 \mathrm{~mL})$. The

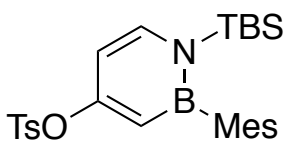
reaction mixture was cooled to $-30{ }^{\circ} \mathrm{C}$. A solution of 4-methylbenzenesulfonyl chloride $(0.93 \mathrm{~g}, 4.89 \mathrm{mmol})$ in $\mathrm{CH}_{2} \mathrm{Cl}_{2}(2.0 \mathrm{~mL})$ was then added. The reaction mixture was allowed to warm to room temperature over a period of one hour. The reaction mixture was then removed from the glovebox and washed with aqueous $\mathrm{NaHCO}_{3}$. The organic layer was extracted with excess ether, dried over $\mathrm{MgSO}_{4}$, and passed through a Buchner funnel lined with filter paper. Volatiles were removed under reduced pressure, and the desired product was obtained as a white solid after purification by silica gel chromatography with $9: 1$ pentane:ether as the eluent $(1.76 \mathrm{~g}$, 60\%). ${ }^{1} \mathrm{H}$ NMR $\left(500 \mathrm{MHz}, \mathrm{CD}_{2} \mathrm{Cl}_{2}\right) \delta 7.70(\mathrm{~d}, J=7.8 \mathrm{~Hz}, 2 \mathrm{H}), 7.48(\mathrm{~d}, J=7.3 \mathrm{~Hz}$, $1 \mathrm{H}), 7.28(\mathrm{~d}, J=8.0 \mathrm{~Hz}, 2 \mathrm{H}), 6.73(\mathrm{~s}, 2 \mathrm{H}), 6.25(\mathrm{dd}, J=7.4,3.0 \mathrm{~Hz}, 1 \mathrm{H}), 5.84(\mathrm{~d}, J=$ $3.0 \mathrm{~Hz}, 1 \mathrm{H}), 2.40(\mathrm{~s}, 3 \mathrm{H}), 2.24(\mathrm{~s}, 3 \mathrm{H}), 1.92(\mathrm{~s}, 6 \mathrm{H}), 0.86(\mathrm{~s}, 9 \mathrm{H}),-0.03(\mathrm{~s}, 6 \mathrm{H}) .{ }^{13} \mathrm{C}$ NMR $\left(126 \mathrm{MHz}, \mathrm{CD}_{2} \mathrm{Cl}_{2}\right) \delta 159.38,145.30,142.25,138.47,136.72,129.74,129.52$, $128.36,126.77,106.99,26.95,22.84,21.29,20.76,18.90,-3.75$. (the carbons adjacent to boron were not observed). ${ }^{11} \mathrm{~B}$ NMR $\left(160 \mathrm{MHz}, \mathrm{CD}_{2} \mathrm{Cl}_{2}\right) \delta 41.41$. FTIR (thin film): $\tilde{v}=2960,2930,2860,1607,1521$. HRMS (DART) calcd. for $\mathrm{C}_{26} \mathrm{H}_{37} \mathrm{BNO}_{3} \mathrm{SSi}$ $\left([\mathrm{M}+\mathrm{H}]^{+}\right)$482.23617, found 482.23618.

Compound 4ptol (from 4Bpin). A $4 \mathrm{~mL}$ pressure vessel was charged with 4Bpin (200 mg, $0.46 \mathrm{mmol}$ ), 4bromotoluene $(78 \mathrm{mg}, 0.46 \mathrm{mmol}), \mathrm{Pd}(\mathrm{dppf}) \mathrm{Cl}_{2}(16 \mathrm{mg}$, $0.023 \mathrm{mmol}), \mathrm{KOH}(77 \mathrm{mg}, 1.37 \mathrm{mmol}), \mathrm{H}_{2} \mathrm{O}(0.2 \mathrm{~mL})$, and THF $(2.0 \mathrm{~mL})$. The $\mathrm{KOH}$ pellets were ground into a<smiles>CB1C=C(c2ccc(C)cc2)C=CN1[13CH3]</smiles>
powder and the $\mathrm{H}_{2} \mathrm{O}$ was sparged with argon for 1 hour prior to use. The reaction mixture was then heated at $80^{\circ} \mathrm{C}$ for 2.5 hours. The organic layer was decanted, and the reaction mixture was concentrated under reduced pressure. The desired product was obtained as a white solid after purification by silica gel chromatography with 99:1 pentane:ether as the eluent (149 mg, 81\%).

Compound 4ptol (from 4OTs). A $4 \mathrm{~mL}$ pressure vessel was charged with 4OTs (100 $\mathrm{mg}, 0.21 \mathrm{mmol}), p$-tolylmagnesium bromide $1.0 \mathrm{M}$ THF solution $(0.415 \mathrm{~mL}, 0.415$ mmol), $\mathrm{Pd}_{2} \mathrm{dba}_{3}$ (5.0 mg, $0.0050 \mathrm{mmol}$ ), 4,4,5,5-tetramethyl-1,3,2-dioxaphospholane2-oxide $(2.0 \mathrm{mg}, 0.010 \mathrm{mmol})$, and 1,4-dioxane $(1.0 \mathrm{~mL})$. The reaction mixture was then heated at $80{ }^{\circ} \mathrm{C}$ for 22 hours. At the conclusion of the reaction, the reaction mixture was concentrated under reduced pressure. The desired product was obtained as a white solid after purification by silica gel chromatography with 99:1 pentane:ether as the eluent (64 $\mathrm{mg}, 76 \%$ ).

${ }^{1} \mathrm{H}$ NMR (500 MHz, $\left.\mathrm{CD}_{2} \mathrm{Cl}_{2}\right) \delta 7.56(\mathrm{~m}, 3 \mathrm{H}), 7.22(\mathrm{~d}, J=7.9 \mathrm{~Hz}, 2 \mathrm{H}), 6.79(\mathrm{~s}, 2 \mathrm{H})$, $6.76(\mathrm{~s}, 1 \mathrm{H}), 6.70(\mathrm{~d}, J=7.0 \mathrm{~Hz}, 1 \mathrm{H}), 2.37(\mathrm{~s}, 3 \mathrm{H}), 2.28(\mathrm{~s}, 3 \mathrm{H}), 2.11(\mathrm{~s}, 6 \mathrm{H}), 0.94(\mathrm{~s}$, $9 \mathrm{H}), 0.04(\mathrm{~s}, 6 \mathrm{H}) .{ }^{13} \mathrm{C}$ NMR $\left(126 \mathrm{MHz}, \mathrm{CD}_{2} \mathrm{Cl}_{2}\right) \delta 153.09,139.39,138.75,138.70$, $138.01,136.21,129.17,126.73,126.53,110.48,27.16,23.19,20.82,20.8,19.07$, 3.64. (the carbons adjacent to boron were not observed). ${ }^{11} \mathrm{~B}$ NMR (160 MHz, $\left.\mathrm{CD}_{2} \mathrm{Cl}_{2}\right) \delta$ 37.07. FTIR (thin film): $\tilde{v}=2961,2930,2859,1611,1570$. HRMS (DART) calcd. for $\mathrm{C}_{26} \mathrm{H}_{36} \mathrm{BNSi}\left([\mathrm{M}+\mathrm{H}]^{+}\right)$402.27828, found 402.27944. 
Compound 5I. A $100 \mathrm{~mL}$ round bottom flask was charged with THF $(10 \mathrm{~mL})$ and cooled to $-78^{\circ} \mathrm{C}$. $n$-Butyllithium $2.5 \mathrm{M}$ hexanes solution $(0.89 \mathrm{~mL}, 2.2 \mathrm{mmol})$ was added via syringe. 5Bpin $(750$ $\mathrm{mg}, 1.72 \mathrm{mmol})$ was dissolved in THF $(5.0 \mathrm{~mL})$ and added<smiles>C[13CH2]N1C=C(I)C=CB1[131I]</smiles>
dropwise via syringe to the flask containing $n$-butyllithium at $-78{ }^{\circ} \mathrm{C}$. The reaction mixture was allowed to warm to room temperature, whereupon the formation of the borate was confirmed by ${ }^{11} \mathrm{~B}$ NMR $(\delta 2.83)$. The reaction mixture was again cooled to $-78^{\circ} \mathrm{C}$. $\mathrm{I}_{2}(1.75 \mathrm{~g}, 6.88 \mathrm{mmol})$ was dissolved in methanol $(10 \mathrm{~mL})$ and added via syringe to the flask containing the borate. The reaction mixture was allowed to stir at $-78^{\circ} \mathrm{C}$ for 30 minutes, then the mixture was allowed to warm to room temperature over a period of 1 hour. At the conclusion of the reaction, the reaction mixture was quenched with aqueous $\mathrm{Na}_{2} \mathrm{SO}_{3}$ and then extracted with hexanes. The organic layer was then washed with brine. Finally, the organic layer was dried over $\mathrm{MgSO}_{4}$, passed through a Buchner funnel lined with filter paper, and concentrated in vacuo. The desired product was obtained as a highly viscous yellow oil after purification by silica gel chromatography with hexanes as the eluent $(676 \mathrm{mg}, 90 \%) .{ }^{1} \mathrm{H}$ NMR $(500 \mathrm{MHz}$, $\left.\mathrm{C}_{6} \mathrm{D}_{6}\right) \delta 7.84(\mathrm{~s}, 1 \mathrm{H}), 7.70(\mathrm{dd}, J=11.5,1.7 \mathrm{~Hz}, 1 \mathrm{H}), 6.79(\mathrm{~s}, 2 \mathrm{H}), 6.53(\mathrm{~d}, J=11.5$ $\mathrm{Hz}, 1 \mathrm{H}), 2.22(\mathrm{~s}, 3 \mathrm{H}), 2.12(\mathrm{~s}, 6 \mathrm{H}), 0.75(\mathrm{~s}, 9 \mathrm{H}),-0.14(\mathrm{~s}, 6 \mathrm{H}) .{ }^{13} \mathrm{C}$ NMR $(126 \mathrm{MHz}$, $\left.\mathrm{CD}_{2} \mathrm{Cl}_{2}\right) \delta 150.01,143.41,138.57,136.63,126.83,109.99,26.96,23.07,20.80,18.96$, -3.75. (the carbons adjacent to boron were not observed). ${ }^{11} \mathrm{~B}$ NMR (160 MHz, $\mathrm{C}_{6} \mathrm{D}_{6}$ ) $\delta$ 38.96. FTIR (thin film): $\tilde{v}=2959,2930,2859,1609,1584$. HRMS (DART) calcd. for $\mathrm{C}_{19} \mathrm{H}_{30} \mathrm{BNSiI}\left([\mathrm{M}+\mathrm{H}]^{+}\right) 438.1285$, found 438.1281 .

Compound 5ptol (From 5Bpin). A $4 \mathrm{~mL}$ pressure vessel was charged with 5Bpin (200 $\mathrm{mg}, 0.46 \mathrm{mmol})$, 4bromotoluene $(78 \mathrm{mg}, 0.46 \mathrm{mmol}), \mathrm{Pd}(\mathrm{dppf}) \mathrm{Cl}_{2}(16 \mathrm{mg}$, $0.023 \mathrm{mmol}), \mathrm{KOH}(77 \mathrm{mg}, 1.37 \mathrm{mmol}), \mathrm{H}_{2} \mathrm{O}(0.2 \mathrm{~mL})$, and THF $(2.0 \mathrm{~mL})$. The $\mathrm{KOH}$ pellets were ground into a powder<smiles></smiles>
and the $\mathrm{H}_{2} \mathrm{O}$ was sparged with argon for 1 hour prior to use. The reaction mixture was then heated at $80{ }^{\circ} \mathrm{C}$ for 2.5 hours. The organic layer was decanted, and the reaction mixture was concentrated under reduced pressure. The desired product was obtained as a white solid after purification by silica gel chromatography with 99:1 pentane:ether as the eluent (152 mg, 82\%).

Compound 5ptol (from 5I). A $4 \mathrm{~mL}$ vial was charged with $5 \mathrm{I}$ ( $85 \mathrm{mg}, 0.19 \mathrm{mmol})$, $p$ tolylboronic acid $(27 \mathrm{mg}, 0.19 \mathrm{mmol}), \mathrm{Pd}(\mathrm{dppf}) \mathrm{Cl}_{2}(7 \mathrm{mg}, 0.010 \mathrm{mmol}), \mathrm{KOH}(33$ $\mathrm{mg}, 0.58 \mathrm{mmol}), \mathrm{H}_{2} \mathrm{O}(0.15 \mathrm{~mL})$, and THF $(1.5 \mathrm{~mL})$. The $\mathrm{KOH}$ pellets were ground into a powder and the $\mathrm{H}_{2} \mathrm{O}$ was sparged with argon for 1 hour prior to use. The reaction mixture was then allowed to stir at room temperature 1.5 hours. The organic layer was decanted, and the reaction mixture was concentrated under reduced pressure. The desired product was obtained as a white solid after purification by silica gel chromatography with 99:1 pentane:ether as the eluent (61 mg, 78\%).

${ }^{1} \mathrm{H}$ NMR $\left(500 \mathrm{MHz}, \mathrm{CD}_{2} \mathrm{Cl}_{2}\right) \delta 7.87(\mathrm{dd}, J=11.2,1.8 \mathrm{~Hz}, 1 \mathrm{H}), 7.67(\mathrm{~d}, J=1.8 \mathrm{~Hz}$, $1 \mathrm{H}), 7.38(\mathrm{~d}, J=8.0 \mathrm{~Hz}, 2 \mathrm{H}), 7.23(\mathrm{~d}, J=7.8 \mathrm{~Hz}, 2 \mathrm{H}), 6.79$ (s, 2H), $6.66(\mathrm{~d}, J=11.2$ $\mathrm{Hz}, 1 \mathrm{H}), 2.37(\mathrm{~s}, 3 \mathrm{H}), 2.28(\mathrm{~s}, 3 \mathrm{H}), 2.10(\mathrm{~s}, 6 \mathrm{H}), 0.95(\mathrm{~s}, 9 \mathrm{H}), 0.05(\mathrm{~s}, 6 \mathrm{H}) .{ }^{13} \mathrm{C} \mathrm{NMR}$ $\left(126 \mathrm{MHz}, \mathrm{CD}_{2} \mathrm{Cl}_{2}\right) \delta 143.19,138.69,137.75$ 136.76, 136.30, 135.89, 129.39, 126.75, $126.12,124.76,27.25,23.17,20.82,20.67,19.02,-3.48$. (the carbons adjacent to boron were not observed). ${ }^{11} \mathrm{~B}$ NMR $\left(160 \mathrm{MHz}, \mathrm{CD}_{2} \mathrm{Cl}_{2}\right) \delta 36.55$. FTIR (thin film): $\tilde{v}$ $=2960,2930,2959,1613,1558$. HRMS (DART) calcd. for $\mathrm{C}_{26} \mathrm{H}_{36} \mathrm{BNSi}\left([\mathrm{M}+\mathrm{H}]^{+}\right)$ 402.27828, found 402.27851. 


\section{Kinetic and Thermodynamic Experiments}

\subsection{Rate Determination of NMO Oxidation by ${ }^{1} \mathrm{H}$ NMR}

\section{General Experimental Procedure for ${ }^{1} \mathrm{H}$ NMR}

In a glovebox, a J-Young tube was charged with $250 \mu \mathrm{L}$ of a stock solution in $\mathrm{CD}_{2} \mathrm{Cl}_{2}$ containing both: 1) internal standard 1,3,5-trimethoxybenzene (stock solution: 24.7 mg in $2.0 \mathrm{~mL} ; 250 \mu \mathrm{L}$ aliquot: $3.1 \mathrm{mg}, 0.018 \mathrm{mmol}$ ) and 2) 5Bpin, 4Bpin, or PhBpin $\left(250 \mu \mathrm{L}\right.$ aliquot from a $0.128 \mathrm{M}$ solution: $0.032 \mathrm{mmol}$ ) and $100 \mu \mathrm{L}$ of $\mathrm{CD}_{2} \mathrm{Cl}_{2}$. Immediately prior to collecting the ${ }^{1} \mathrm{H}$ NMR spectrum, $250 \mu \mathrm{L}$ of a stock solution in $\mathrm{CD}_{2} \mathrm{Cl}_{2}$ containing $\mathrm{NMO}$ (stock solution: $240 \mathrm{mg}$ material in $2.0 \mathrm{~mL} ; 250 \mu \mathrm{L}$ aliquot: $30 \mathrm{mg}, 0.253 \mathrm{mmol}, 8$ equiv.) was added to the J-Young tube. A ${ }^{1} \mathrm{H}$ NMR spectrum was collected every 100 seconds and conversion of the starting material was determined by the internal standard method with a calibration to the measured NMR ratio of the starting material and 1,3,5-trimethoxybenzene in the prepared stock solution. The rate of reaction was determined from the first $10 \%$ of conversion.

Figure S1. 4Bpin oxidation kinetics.
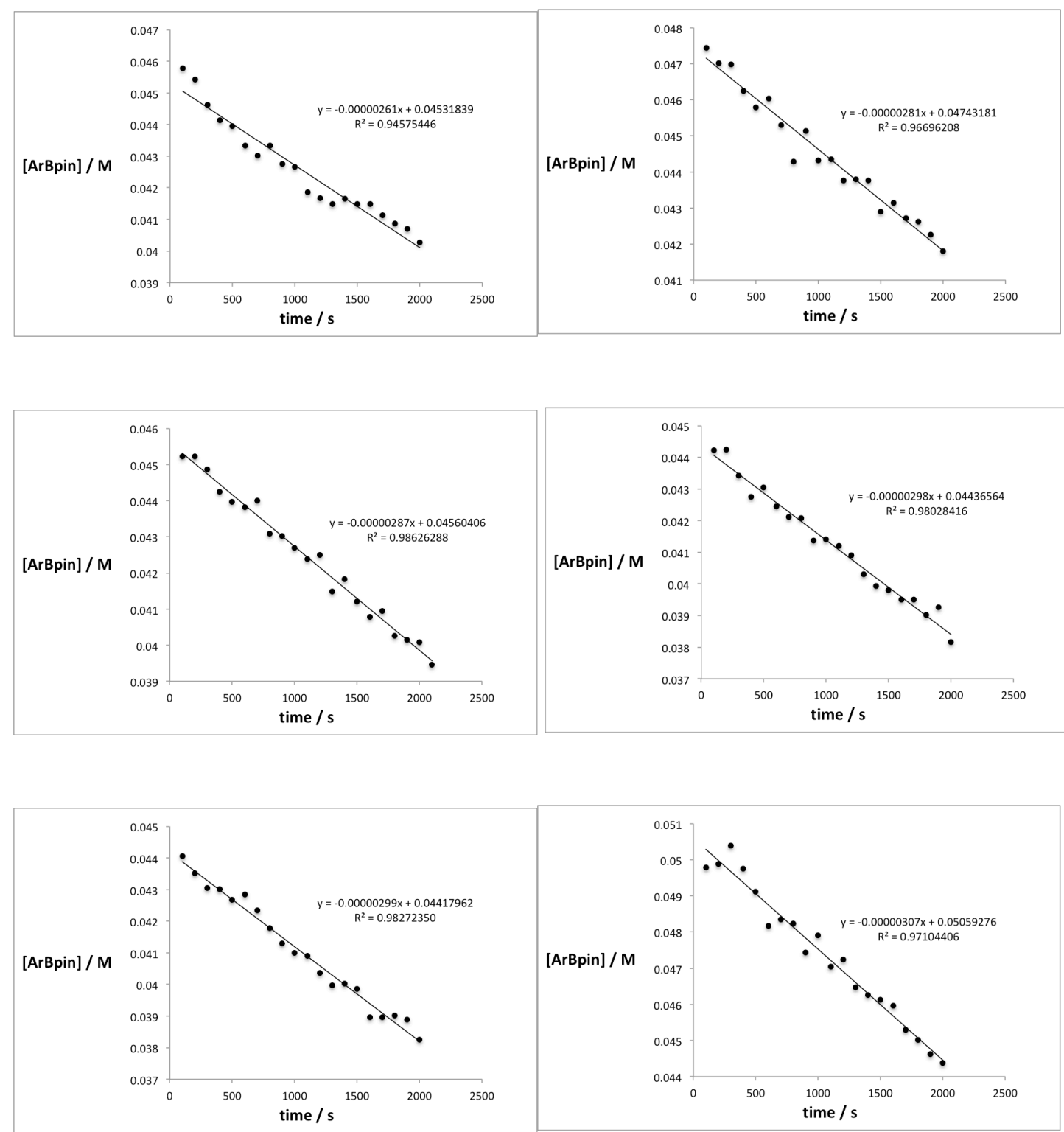
Figure S2. 5Bpin oxidation (decomposition) kinetics.
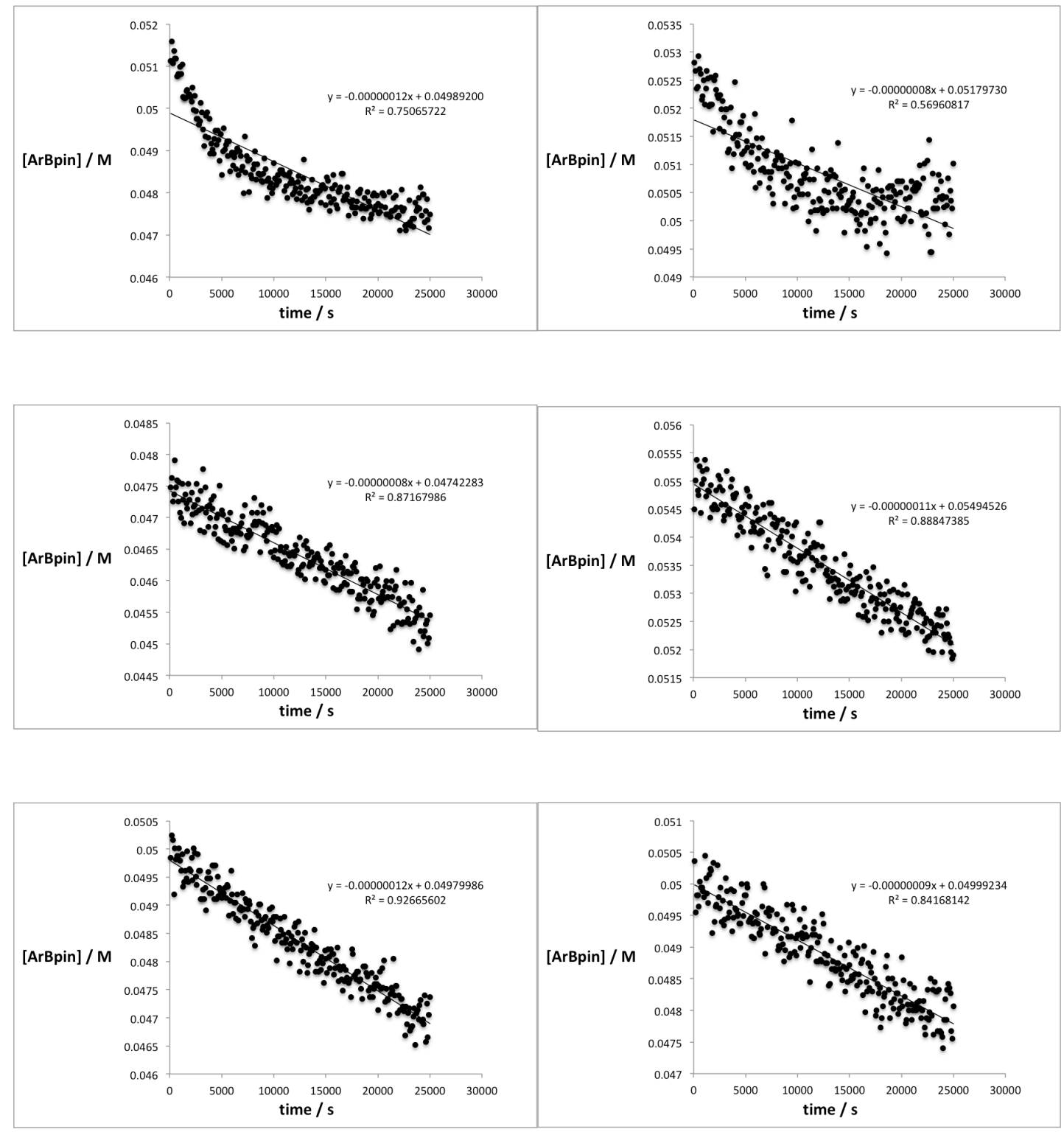
Figure S3. PhBpin Oxidation Kinetics
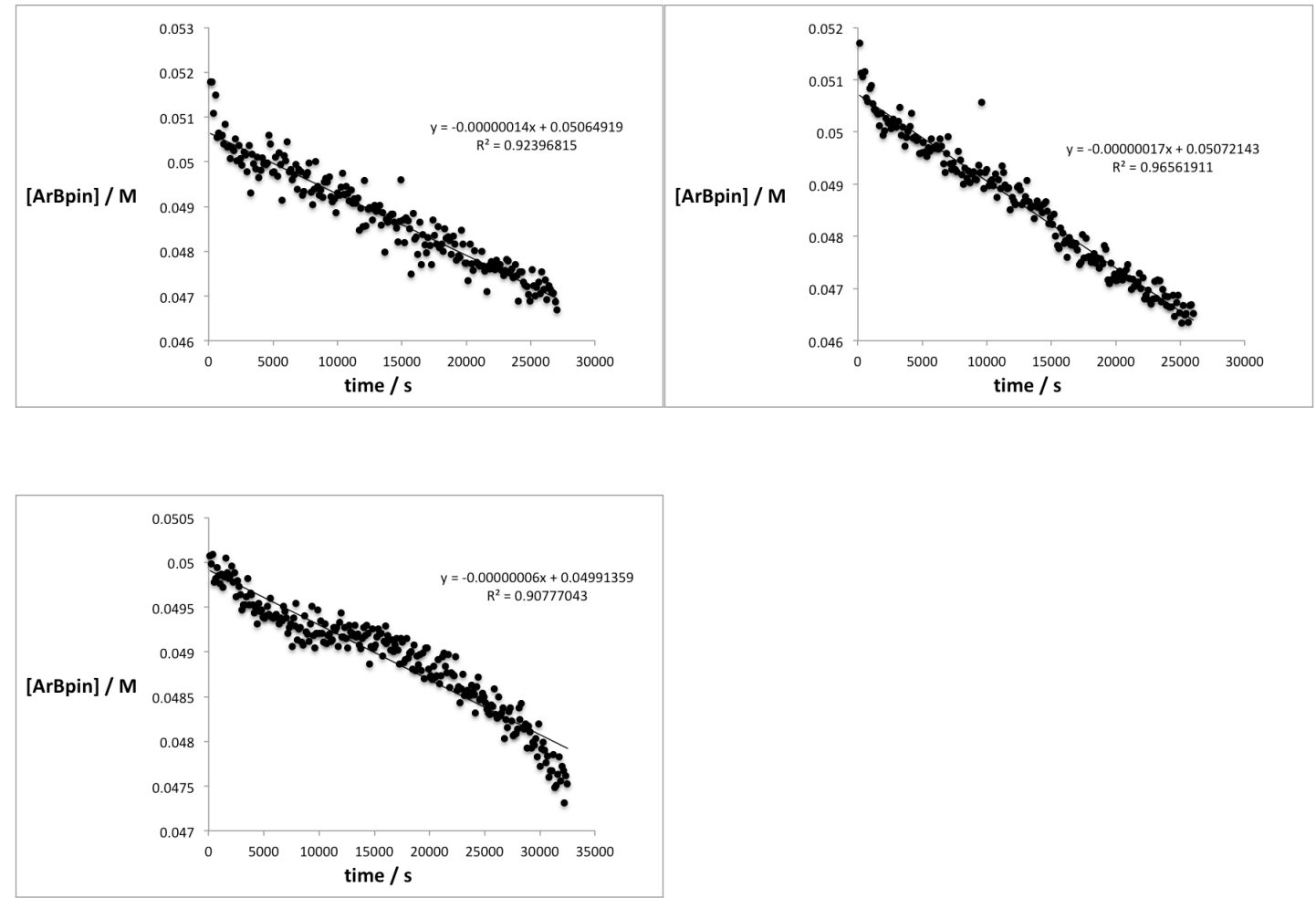

Table S1. Summary of oxidation kinetics.

\begin{tabular}{cccc} 
entry & rate 4Bpin $\left(\mathrm{M} \mathrm{s}^{-1}\right)$ & rate 5Bpin $\left(\mathrm{M} \mathrm{s}^{-1}\right)$ & rate PhBpin $\left(\mathrm{M} \mathrm{s}^{-1}\right)$ \\
\hline 1 & $-2.61 \times 10^{-6}$ & $-1.2 \times 10^{-7}$ & $-1.4 \times 10^{-7}$ \\
2 & $-2.81 \times 10^{-6}$ & $-0.8 \times 10^{-7}$ & $-1.7 \times 10^{-7}$ \\
3 & $-2.87 \times 10^{-6}$ & $-0.8 \times 10^{-7}$ & $-0.6 \times 10^{-7}$ \\
4 & $-2.98 \times 10^{-6}$ & $-1.1 \times 10^{-7}$ & - \\
5 & $-2.99 \times 10^{-6}$ & $-1.2 \times 10^{-7}$ & - \\
6 & $-3.07 \times 10^{-6}$ & $-0.9 \times 10^{-7}$ & - \\
\hline average \pm & $-2.89 \pm 0.16 \times 10^{-6}$ & $-1.0 \pm 0.2 \times 10^{-7}$ & $-1.2 \pm 0.6 \times 10^{-7}$ \\
std. dev. & $\mathbf{2 8 . 9 \pm 5 . 7}$ &
\end{tabular}




\subsection{Rate Determination of Protodeborylation Reactions by ${ }^{1} \mathrm{H}$ NMR}

\section{General Experimental Procedure for ${ }^{1} \mathrm{H}$ NMR}

In a glovebox, a J-Young tube was charged with $250 \mu \mathrm{L}$ of a stock solution in $\mathrm{CD}_{2} \mathrm{Cl}_{2}$ containing 4Bpin or 5Bpin (stock solution: $110 \mathrm{mg}$ material in $2.0 \mathrm{~mL} ; 250 \mu \mathrm{L}$ aliquot: $13.8 \mathrm{mg}$ of material, $0.032 \mathrm{mmol}$ ), $100 \mu \mathrm{L}$ of a stock solution in $\mathrm{CD}_{2} \mathrm{Cl}_{2}$ containing internal standard 1,3,5-trimethoxybenzene (stock solution: $41.6 \mathrm{mg}$ material in $2.0 \mathrm{~mL} ; 100 \mu \mathrm{L}$ aliquot: $5.2 \mathrm{mg}, 0.031 \mathrm{mmol}$ ), and $100 \mu \mathrm{L}$ of a stock solution in $\mathrm{CD}_{2} \mathrm{Cl}_{2}$ containing methanol $(80 \mathrm{mg}$ in $2.0 \mathrm{~mL} ; 10 \mathrm{mg}, 0.31 \mathrm{mmol}$ ), and 50 $\mu \mathrm{L}$ of $\mathrm{CD}_{2} \mathrm{Cl}_{2}$. Immediately prior to collecting the ${ }^{1} \mathrm{H}$ NMR spectrum, $100 \mu \mathrm{L}$ of a stock solution in $\mathrm{CD}_{2} \mathrm{Cl}_{2}$ containing [ $\left.\mathrm{Ir}(\mathrm{OMe})(\operatorname{cod})\right]_{2}(4 \mathrm{mg}$ in $2.0 \mathrm{~mL} ; 0.5 \mathrm{mg}, 0.0008$ mmol) was added to the J-Young tube. The reaction mixture was heated to $40{ }^{\circ} \mathrm{C}$. A ${ }^{1} \mathrm{H}$ NMR spectrum was collected every 80 seconds and conversion of the starting material was determined by the internal standard method with a calibration to the measured NMR ratio of the starting material and 1,3,5-trimethoxybenzene in the prepared stock solution. The rate of reaction was determined from the first $10 \%$ of conversion.

Figure S4. 4Bpin protodeborylation kinetics.
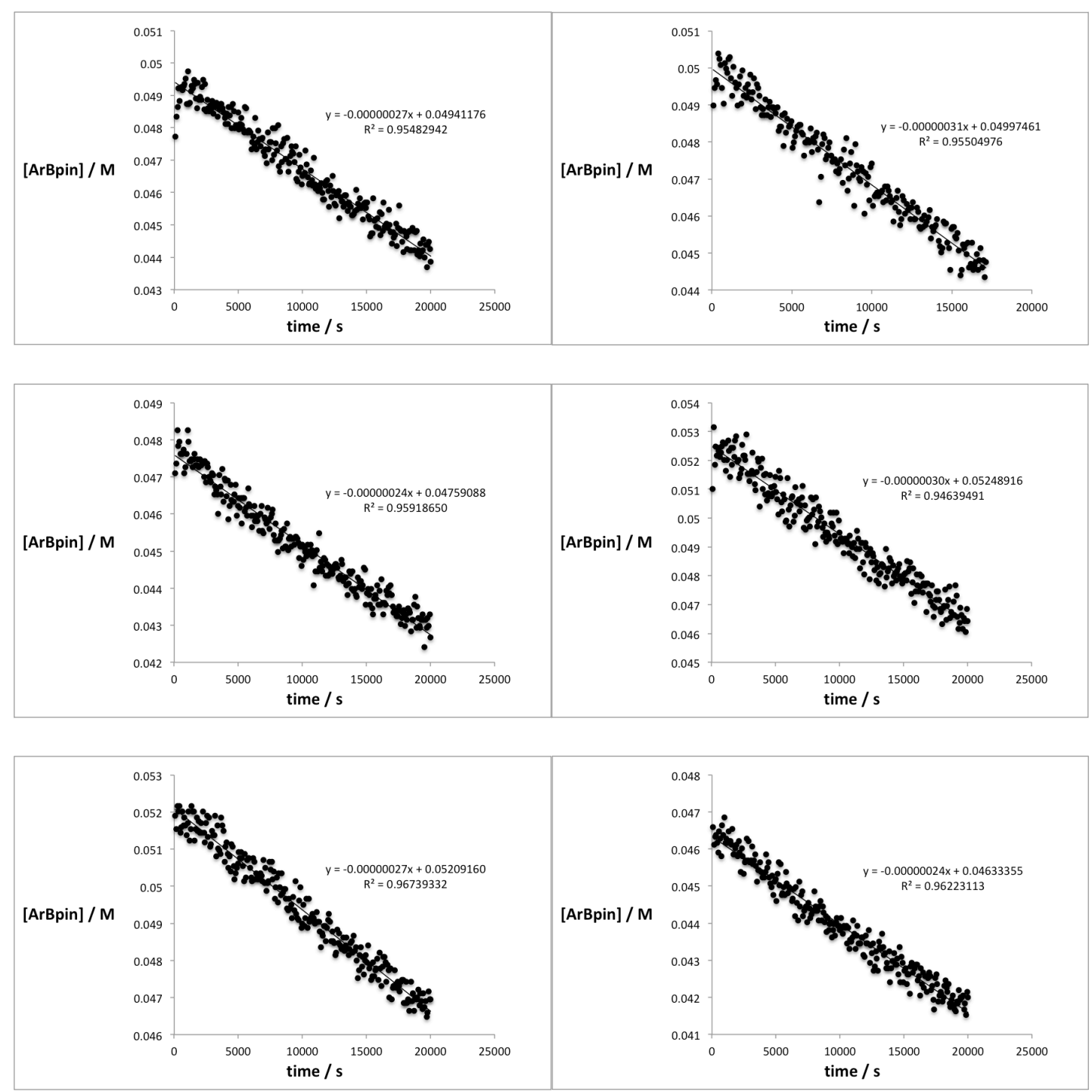
Figure S5. 5Bpin protodeborylation kinetics.
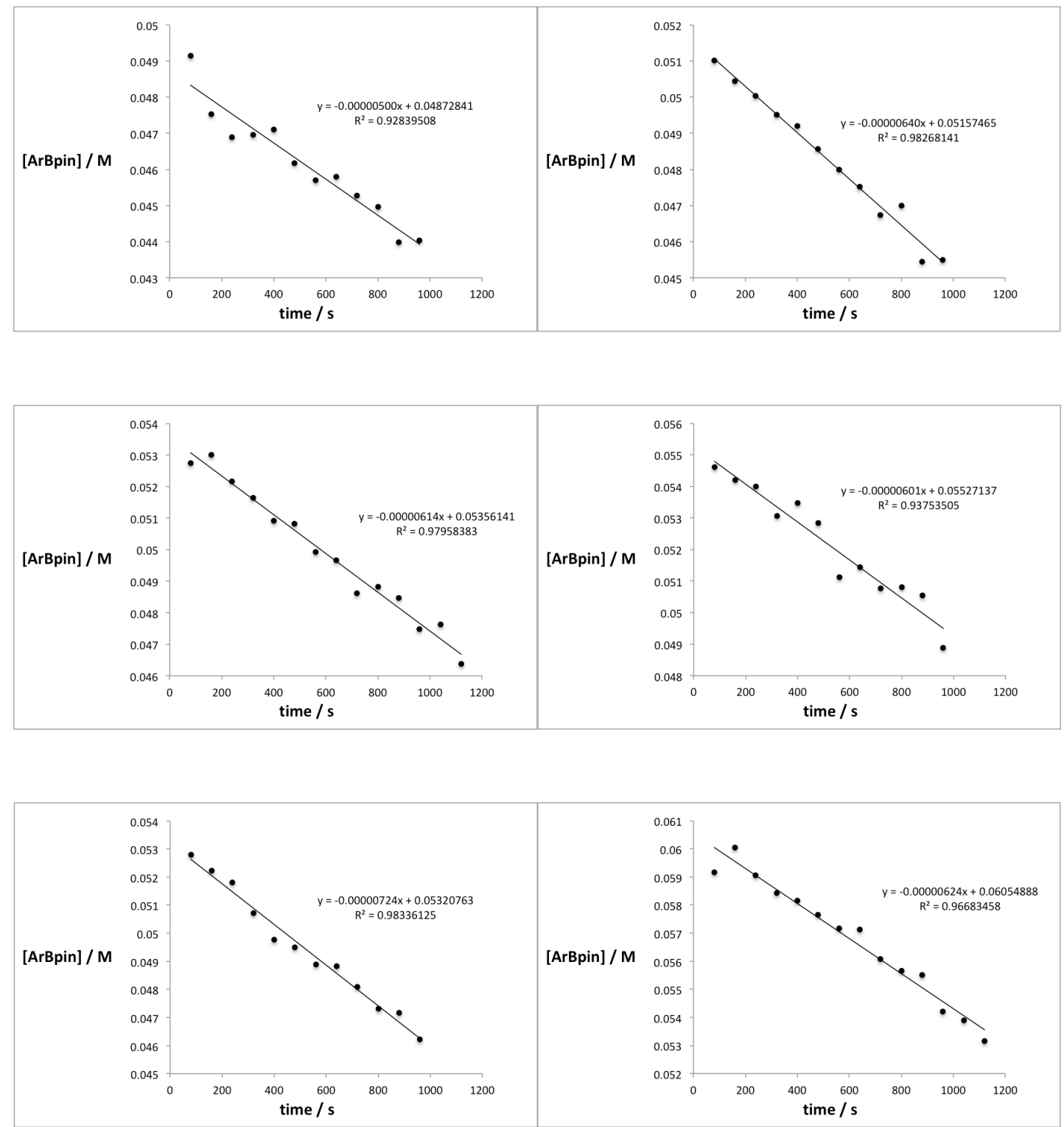
Figure S6. PhBpin protodeborylation kinetics.
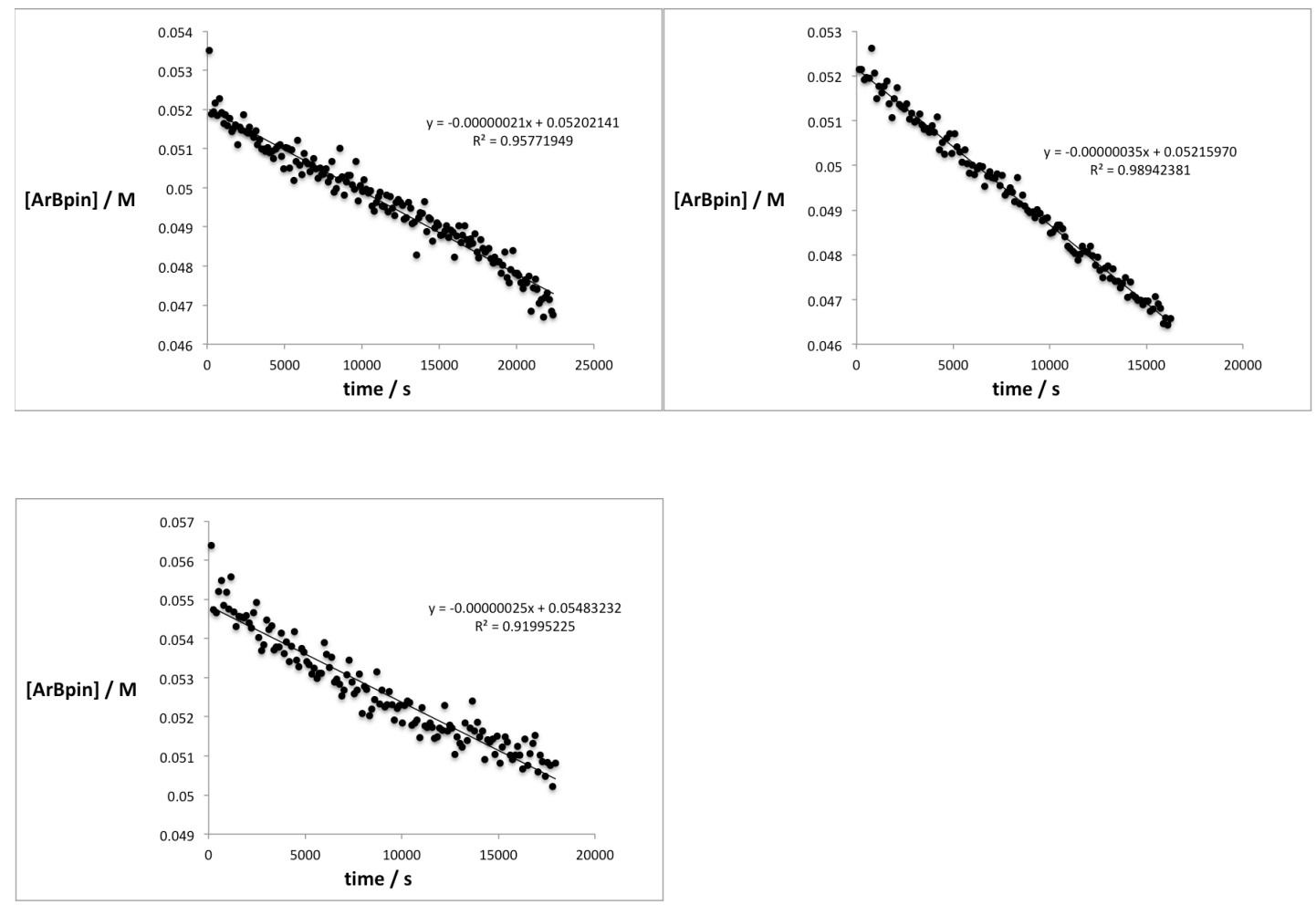

Table S2. Summary of protodeborylation kinetics.

\begin{tabular}{lccc} 
entry & rate 4Bpin $\left(\mathrm{M} \mathrm{s}^{-1}\right)$ & rate 5Bpin $\left(\mathrm{M} \mathrm{s}^{-1}\right)$ & rate PhBpin $\left(\mathrm{M} \mathrm{s}^{-1}\right)$ \\
\hline 1 & $-2.7 \times 10^{-7}$ & $-5.0 \times 10^{-6}$ & $-2.1 \times 10^{-7}$ \\
2 & $-3.1 \times 10^{-7}$ & $-6.4 \times 10^{-6}$ & $-3.5 \times 10^{-7}$ \\
3 & $-2.4 \times 10^{-7}$ & $-6.1 \times 10^{-6}$ & $-2.5 \times 10^{-7}$ \\
4 & $-3.0 \times 10^{-7}$ & $-6.0 \times 10^{-6}$ & - \\
5 & $-2.7 \times 10^{-7}$ & $-7.2 \times 10^{-6}$ & - \\
6 & $-2.4 \times 10^{-7}$ & $-6.2 \times 10^{-6}$ & - \\
\hline $\begin{array}{l}\text { average } \pm \\
\text { std. dev. }\end{array}$ & $-2.7 \pm 0.3 \times 10^{-7}$ & $-6 \pm 1 \times 10^{-6}$ & $-2.7 \pm 0.7 \times 10^{-7}$ \\
\hline rate 5Bpin & $\mathbf{2 2 . 7 \pm 3 . 6}$ & & \\
\hline rate 4Bpin & \multirow{2}{*}{ 4. } & &
\end{tabular}




\subsection{Rate and Equilibrium Constant Determination of Transesterification Reactions}

\section{General Experimental Procedure for Rate Determination with Reaction Calorimetry}

Reactions were performed in a Setaram C80 Calvet calorimeter with Setsoft 2000 software. In a glove box, a stainless steel mixing cell (approx. volume $4.6 \mathrm{~mL}$ ) was charged with $250 \mu \mathrm{L}$ of a stock solution in THF- $\mathrm{D}_{8}$ containing diethanolamine (stock solution: $50.5 \mathrm{mg}$ in $2.0 \mathrm{~mL} ; 250 \mu \mathrm{L}$ aliquot: $6.3 \mathrm{mg}, 0.060 \mathrm{mmol}$ ) and $100 \mu \mathrm{L}$ of THF-D 8 . To the upper chamber was added $250 \mu \mathrm{L}$ of a stock solution in THF-D 8 containing both: 1) internal standard 1,3,5-trimethoxybenzene (101.5 mg : $5.0 \mathrm{~mL}$; $5.1 \mathrm{mg}, 0.030 \mathrm{mmol}$ ) and 2) 4Bpin or 5Bpin (526.5 mg : $5.0 \mathrm{~mL} ; 26 \mathrm{mg}, 0.060$ mmol). The reference cell was left empty under air. Both cells were loaded into the calorimeter at $27^{\circ} \mathrm{C}$ and the heat flow was allowed to equilibrate (approx. 2h). Data collection was initiated, and both plungers were depressed. Heat flow measurements were recorded every 6s. Data collection was halted when heat flow reached equilibrium.

The thermodynamic heat of reaction was obtained through integration of the complete heat flow versus time curve and moles of limiting substrate by equation (1).

$$
\Delta H_{r x n}=\frac{\int_{0}^{\infty} q d t}{m o l}
$$

Heat flow is directly related to rate through equation (2).

$$
\text { heat flow }=\mathrm{q}=\left(\Delta \mathrm{H}_{\mathrm{rxn}}\right)(\text { volume })(\text { rate })
$$

Integration of the heat flow curve from $\mathrm{t}=0$ to $\mathrm{t}=\mathrm{t}$ divided by the complete area under the curve provides conversion (equation (3)).

$$
(\text { Conv })_{t}=\frac{\text { area to time } t}{\text { total area }}=\frac{\int_{0}^{t} q d t}{\int_{0}^{\infty} q d t}
$$

Conversion can also be defined as the ratio of the concentration of starting material at $\mathrm{t}=\mathrm{t}$ relative to its concentration at $\mathrm{t}=0$ (equation (4)).

$$
(\mathrm{Conv})_{t}=\frac{[A]_{t}}{[A]_{0}}
$$

Because the reaction being studied is an equilibrium process, the complete consumption of starting material does not define the endpoint of the reaction as in equation (4). Equation (4) must be modified to equation (5) to define the equilibrium concentration as the new endpoint.

$$
(\text { Conv })_{t e q}=\frac{\left([A]_{t}-[A]_{e q}\right)}{\left([A]_{0}-[A]_{e q}\right)}
$$

Rearranging to express in terms of $[\mathrm{A}]_{\mathrm{t}}$ we obtain equation (6). (Conv) teq is the actual value determined by reaction calorimetry according to equation (3) and $[\mathrm{A}]_{\mathrm{eq}}$ is 
determined by measuring the equilibrium concentration of the starting material (vide infra).

$$
[A]_{t}=(\operatorname{Conv})_{t}\left([A]_{0}-[A]_{e q}\right)+[A]_{e q}
$$

The slope of a plot of $[\mathrm{A}]_{\mathrm{t}}$ versus time over the first $5 \%$ of conversion versus time will give a measure of the instantaneous rate of the reaction.

\section{General Experimental Procedure for Equilibrium Constant Determination with ${ }^{1} \mathbf{H}$ NMR}

The reaction mixture was transferred from the calorimetry cell directly to an NMR tube and analysed by ${ }^{1} \mathrm{H}$ NMR. The concentrations of each component were determined by the internal standard method with a calibration to the measured NMR ratio of each compound and 1,3,5-trimethoxybenzene in the prepared stock solution.

Figure S7. 4Bpin transesterification kinetics.
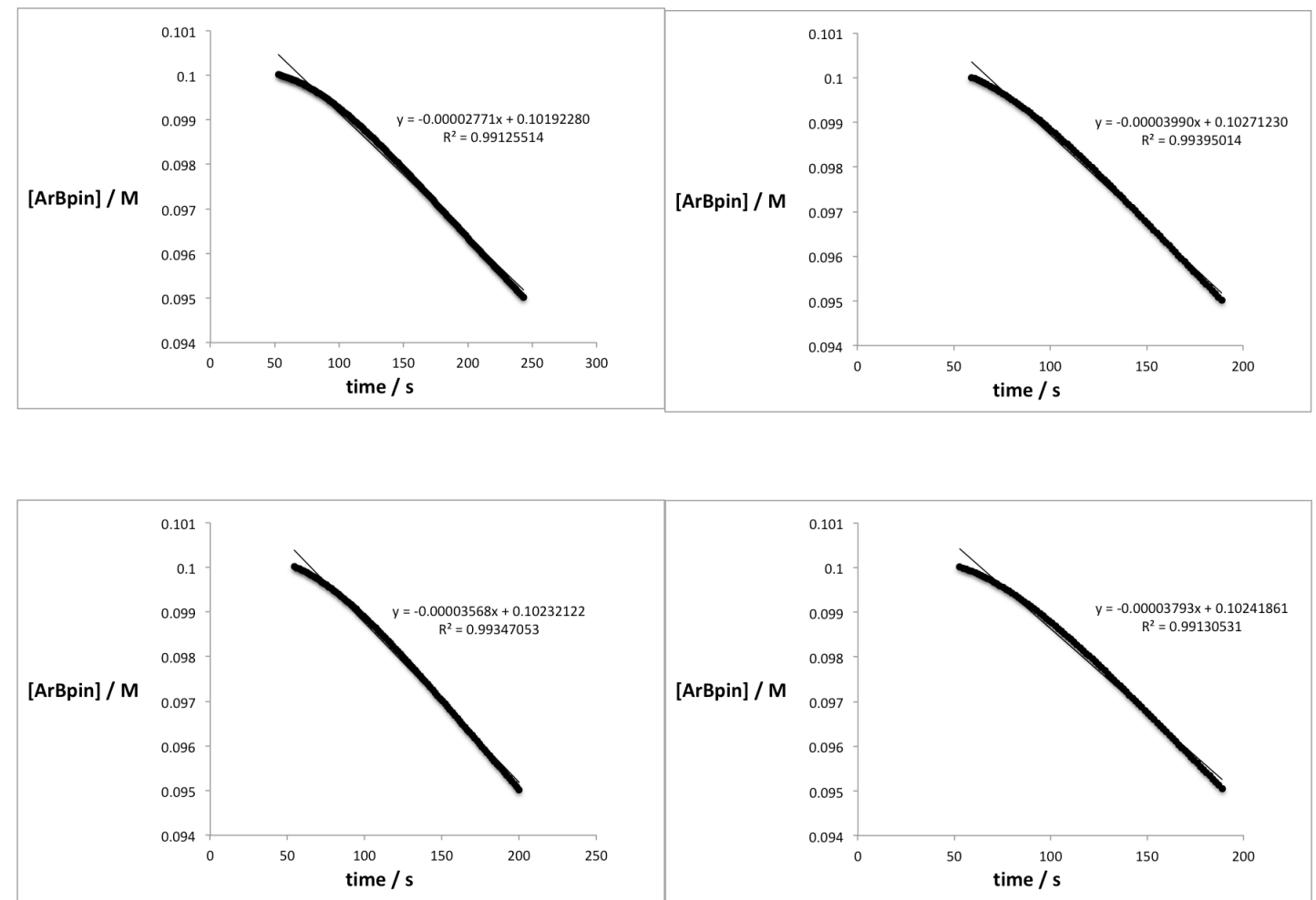
Figure S8. 5Bpin transesterification kinetics.
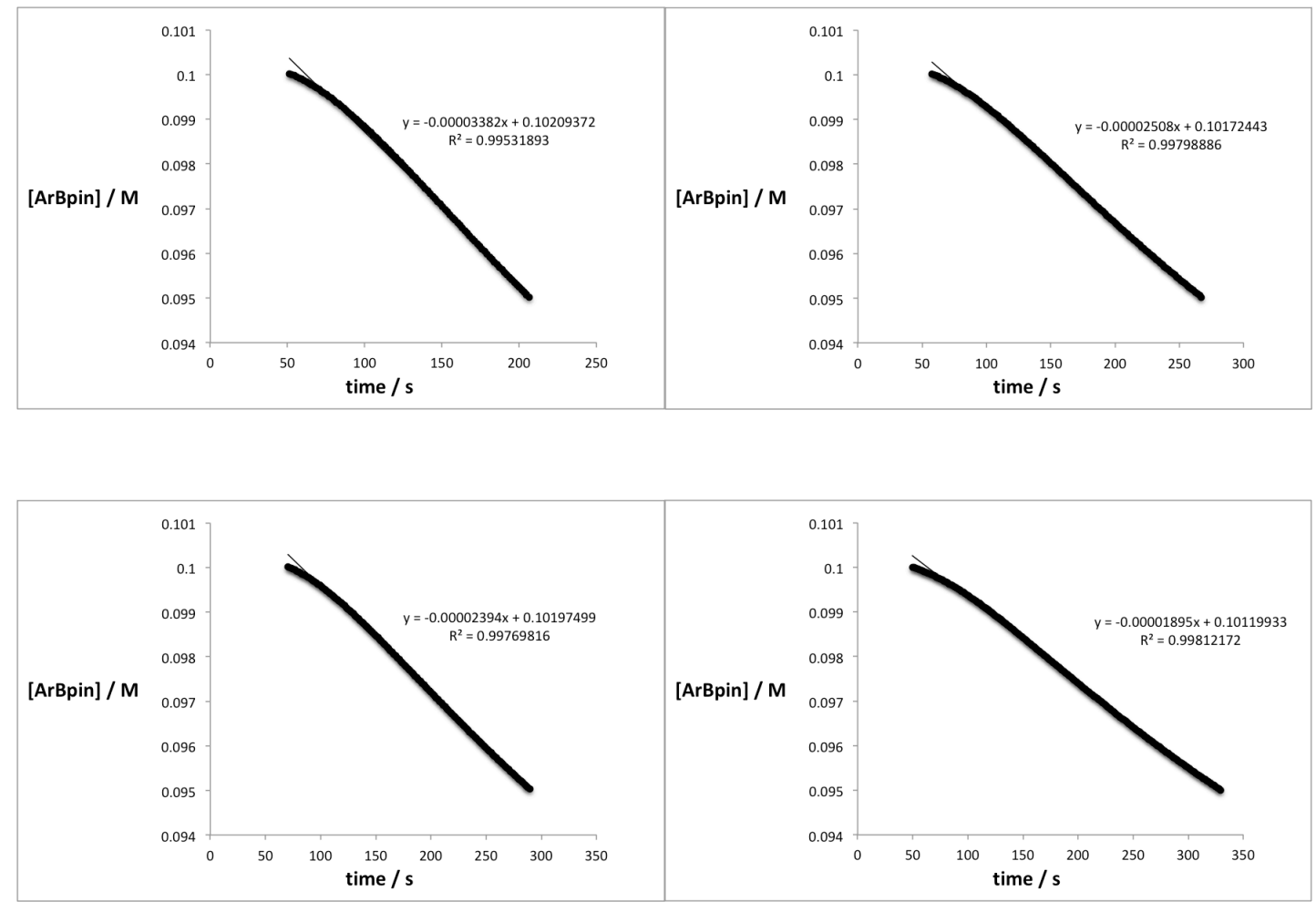

Table S3. Summary of transesterification rates and equilibrium constants.

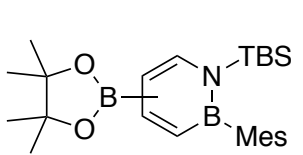

A<smiles>OCCNCCO</smiles>

B

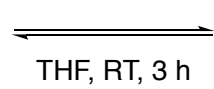

THF, RT, $3 \mathrm{~h}$

$\mathrm{K}_{\mathrm{eq}}=\frac{[\mathrm{C}][\mathrm{D}]}{[\mathrm{A}][\mathrm{B}]}$

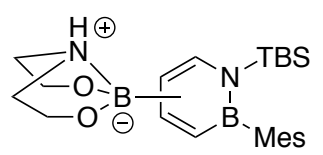

C

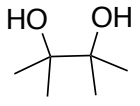

D

entry rate 4Bpin $\left(\mathrm{M} \mathrm{s}^{-1}\right)$ rate 5Bpin $\left(\mathrm{M} \mathrm{s}^{-1}\right) \quad \mathrm{K}_{\mathrm{eq}}$ 4Bpin $\quad \mathrm{K}_{\mathrm{eq}}$ 5Bpin

\begin{tabular}{ccccc}
\hline 1 & $-2.77 \times 10^{-5}$ & $-3.38 \times 10^{-5}$ & 0.22 & 0.011 \\
2 & $-3.99 \times 10^{-5}$ & $-2.51 \times 10^{-5}$ & 0.35 & 0.010 \\
3 & $-3.57 \times 10^{-5}$ & $-2.39 \times 10^{-5}$ & 0.29 & 0.011 \\
4 & $-3.79 \times 10^{-5}$ & $-1.90 \times 10^{-5}$ & 0.30 & 0.009 \\
\hline $\begin{array}{l}\text { average } \pm \\
\text { std. dev. }\end{array}$ & $-3.5 \pm 0.5 \times 10^{-5}$ & $-2.5 \pm 0.6 \times 10^{-5}$ & $0.29 \pm 0.05$ & $0.010 \pm 0.001$ \\
\hline
\end{tabular}

$\frac{\mathrm{K}_{\mathrm{eq}} \text { 4Bpin }}{\mathrm{K}_{\mathrm{eq}} \text { 5Bpin }} \quad 28.6 \pm 6.1$ 


\section{Computational Details}

All calculations were performed using the Gaussian 09 program. ${ }^{2}$

\subsection{Electronic Structure Calculations of 4Bpin and 5Bpin}

Structures 4Bpin and 5Bpin were optimized at the M062X/6-31G(d,p) level and computed using the polarized continuum model simulating $\mathrm{CH}_{2} \mathrm{Cl}_{2}$ solvent. The coordinates can be found in the next section, NMO Oxidation, with the identifiers GS(4Bpin) and GS(5Bpin).

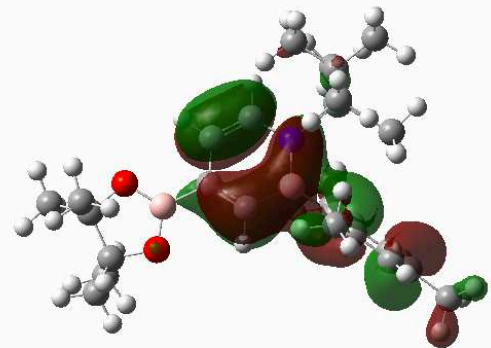

4Bpin HOMO: $-0.2627 \mathrm{eV}$

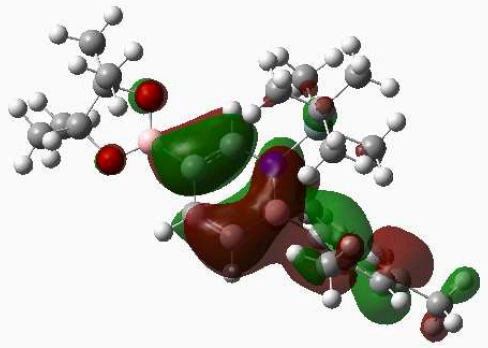

5Bpin HOMO: $-0.2650 \mathrm{eV}$

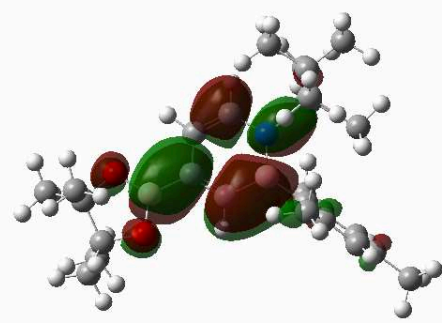

4Bpin LUMO: $-0.00923 \mathrm{eV}$

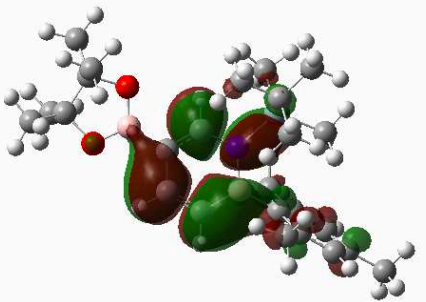

5Bpin LUMO: +0.00882 eV

Figure S9. HOMO and LUMO energies and orbital illustrations of 4Bpin (GS(4Bpin)) and 5Bpin (GS(5Bpin)).

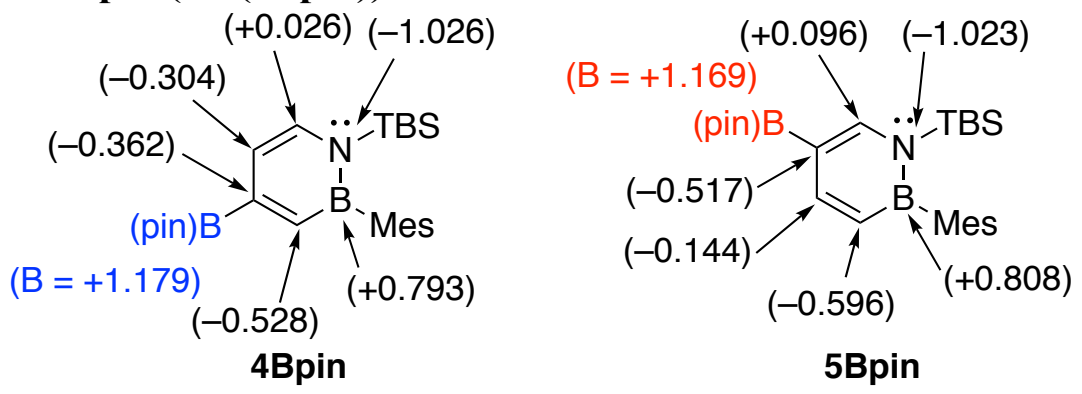

Figure S10. Löwdin charges for selected atoms of 4Bpin (GS(4Bpin)) and 5Bpin (GS(5Bpin)).

\subsection{NMO Oxidation}

All structures for the potential energy surface of the NMO oxidation reaction were optimized at the M062X/6-31G(d,p) level and computed using the polarized continuum model simulating $\mathrm{CH}_{2} \mathrm{Cl}_{2}$ solvent. The coordinates are reproduced here. 
Table S4. Gibbs free energies of structures for the free energy profile for the oxidation of 5Bpin and 4Bpin with NMO. CounterPoise basis set superposition errors (BSSE) are included for selected structures.

\begin{tabular}{lcccc}
\multicolumn{1}{c}{ Structure } & $\begin{array}{c}\text { Gibbs Free Energy } \\
\text { (Hartree) }\end{array}$ & $\begin{array}{c}\text { Gibbs Free Energy } \\
\text { Relative to GS }\left(\mathrm{kcal} \mathrm{mol}^{-1}\right)\end{array}$ & $\begin{array}{c}\text { CounterPoise } \\
\text { BSSE }\left(\mathrm{kcal} \mathrm{mol}^{-1}\right)\end{array}$ & $\begin{array}{c}\text { Corrected Energy Value } \\
\left(\mathrm{kcal} \mathrm{mol}^{-1}\right)\end{array}$ \\
\hline NMO & -401.954235 & - & - & - \\
GS(4Bpin) & -1520.904048 & - & - & - \\
GS(4Bpin) + NMO & -1922.858283 & 0.0 & 7.0 & 12.5 \\
TS1(4Bpin) & -1922.849537 & 5.5 & 8.5 & 2.5 \\
INT(4BPin) & -1922.867865 & -6.0 & 5.5 & 23.8 \\
TS2(4Bpin) & -1922.829616 & 18.2 & - & - \\
PDT(4Bpin) & -1596.142556 & - & - & - \\
NMM & -326.834433 & - & - & -74.5 \\
PDT(4Bpin) + NMM & -1922.976989 & -74.5 & - & - \\
GS(5Bpin) & -1520.91015 & - & - & 0.0 \\
GS(5Bpin) + NMO & -1922.86314 & 0.0 & 6.7 & 10.8 \\
TS1(5Bpin) & -1922.857888 & 4.1 & 8.6 & 3.0 \\
INT(5BPin) & -1922.873288 & -5.6 & 5.6 & 27.7 \\
TS2(5Bpin) & -1922.829203 & 22.1 & - & - \\
PDT(5Bpin) & -1596.131506 & -63.7 & - & -63.7 \\
PDT(5Bpin) + NMM & -1922.965939 & & -6 & -1
\end{tabular}

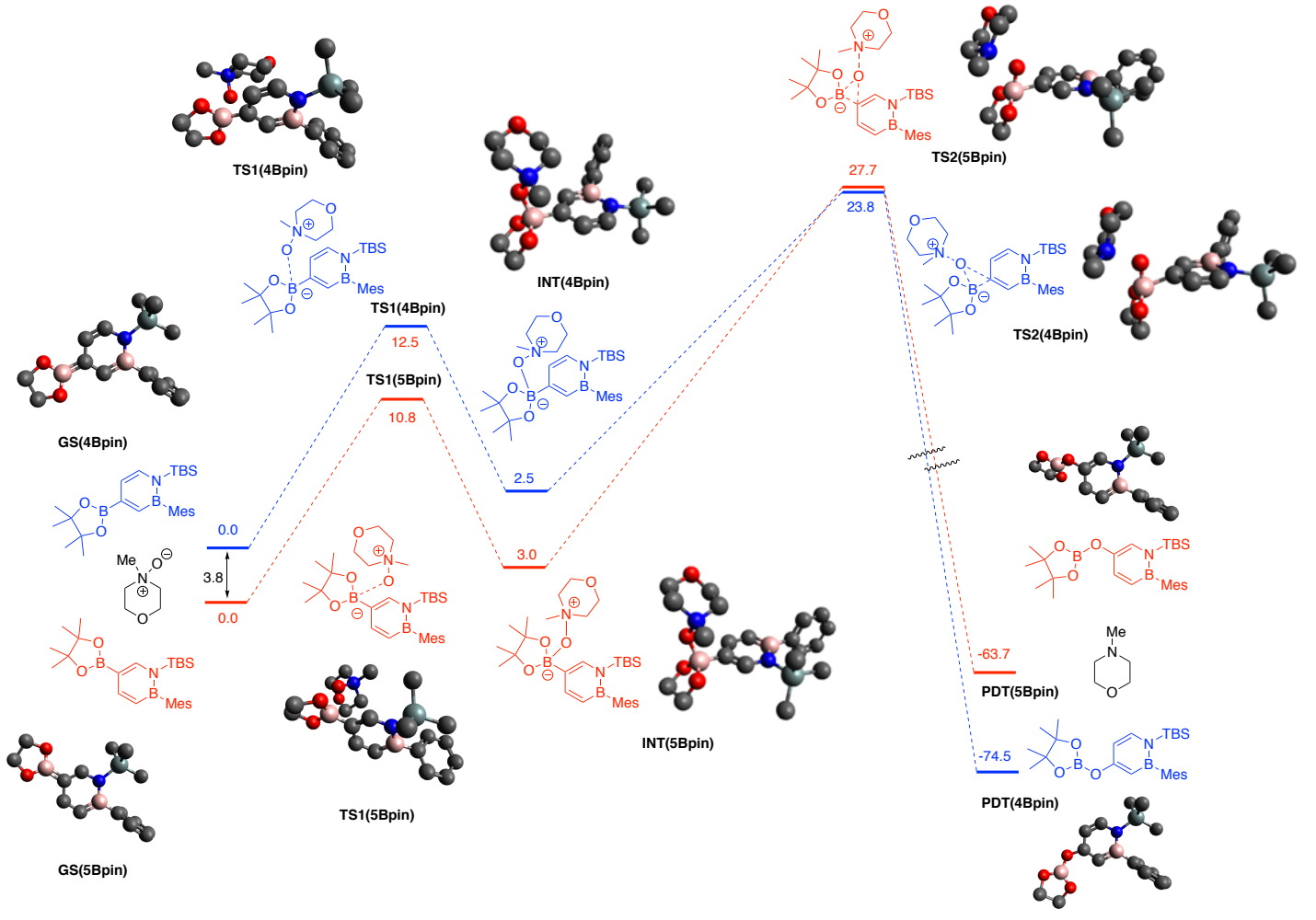

Figure S11. Calculated free energy profile (in $\mathrm{kcal} / \mathrm{mol}$ ) for the oxidation of 4Bpin and 5Bpin with NMO. $\mathrm{H}$ atoms as well as methyl-carbons on the mesityl, pinacol, and tert-butyl groups omitted for clarity. 
Figure S12. Reaction coordinates for optimized geometries for NMO oxidation.

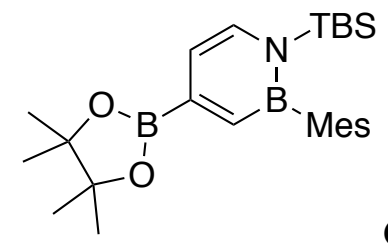
B $-3.461-0.300 \quad 0.014$
$\begin{array}{llll}\text { O } & -4.421 & -1.267 & 0.168\end{array}$
C $-5.690-0.658-0.172$
C $-6.779-1.258 \quad 0.700$
H $-6.527-1.184 \quad 1.759$

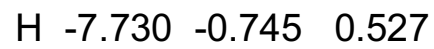

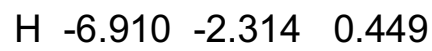
$\begin{array}{llll}C & -5.406 & 0.858 & 0.085\end{array}$
C $-5.611 \quad 1.258 \quad 1.543$

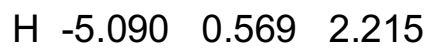
H $-6.672 \quad 1.269 \quad 1.806$
$\begin{array}{llll}H & -5.203 & 2.260 & 1.695\end{array}$
$\begin{array}{llll}C & -6.146 & 1.810 & -0.839\end{array}$
$\begin{array}{llll}\mathrm{H} & -5.886 & 2.841 & -0.586\end{array}$
$\begin{array}{llll}H & -7.228 & 1.691 & -0.718\end{array}$
H $-5.887 \quad 1.635-1.884$
C $-5.949-0.972-1.644$
H $-5.931-2.056-1.781$
$\begin{array}{llll}\mathrm{H} & -6.923 & -0.596 & -1.966\end{array}$
H $-5.175 \quad-0.531-2.279$
$\begin{array}{llll}C & -1.927 & -0.604 & 0.051\end{array}$
$\begin{array}{llll}C & -1.483 & -1.953 & 0.216\end{array}$
$\begin{array}{llll}\mathrm{H} & -2.205 & -2.754 & 0.329\end{array}$
$\begin{array}{llll}\text { C } & -0.157 & -2.259 & 0.234\end{array}$
$\begin{array}{llll}\text { H } & 0.152 & -3.293 & 0.359\end{array}$
$\begin{array}{llll}N & 0.853 & -1.324 & 0.104\end{array}$
Si $2.511-2.059-0.029$
$\begin{array}{llll}\text { C } & 3.915 & -0.845 & 0.234\end{array}$
$\begin{array}{llll}\text { H } & 3.946 & -0.042 & -0.506\end{array}$
$\begin{array}{llll}\text { H } & 4.846 & -1.418 & 0.168\end{array}$
H $3.878-0.385 \quad 1.224$
C $2.661-2.834-1.762$
$\begin{array}{llll}C & 2.570 & -1.731 & -2.824\end{array}$
$\begin{array}{llll}\text { H } & 3.349 & -0.972 & -2.694\end{array}$
$\begin{array}{llll}\text { H } & 1.597 & -1.228 & -2.787\end{array}$
H $2.687 \quad-2.160 \quad-3.827$
$\begin{array}{llll}\text { C } & 4.030 & -3.528 & -1.859\end{array}$
$\begin{array}{llll}\text { H } & 4.858 & -2.823 & -1.731\end{array}$
H $4.143 \quad-3.991-2.848$
H $4.138-4.321-1.110$
$\begin{array}{llll}\text { C } & 1.564 & -3.872 & -2.036\end{array}$
$\begin{array}{llll}\text { H } & 0.567 & -3.420 & -2.043\end{array}$
$\begin{array}{llll}\text { H } & 1.727 & -4.328 & -3.021\end{array}$
H 1.568 -4.681 -1.296
$\begin{array}{lll}\text { C } 2.631 & -3.374 & 1.312\end{array}$
H $3.682-3.646 \quad 1.456$
$\begin{array}{llll}H & 2.255 & -2.985 & 2.264\end{array}$ 

H $2.082 \quad-4.292 \quad 1.082$
$\begin{array}{llll}\text { B } & 0.501 & 0.079 & -0.015\end{array}$
C $1.5831 .234 \quad 0.016$
C $2.120 \quad 1.616 \quad 1.268$
$\begin{array}{llll}C & 1.684 & 0.904 & 2.528\end{array}$
H $2.115 \quad 1.372 \quad 3.416$
$\begin{array}{llll}H & 0.594 & 0.912 & 2.630\end{array}$
H $1.989-0.149 \quad 2.521$
C $3.053 \quad 2.643 \quad 1.346$
H $3.4592 .923 \quad 2.317$
C $3.481 \quad 3.330 \quad 0.204$
C $4.490 \quad 4.444 \quad 0.315$
H $\begin{array}{lll}5.365 & 4.127 & 0.890\end{array}$
H $4.062 \quad 5.311 \quad 0.828$
H $4.830 \quad 4.771 \quad-0.670$
C $2.924 \quad 2.974 \quad-1.019$
H $3.224 \quad 3.511 \quad-1.917$
C $1.973 \quad 1.950-1.125$
$\begin{array}{llll}C & 1.395 & 1.653 & -2.489\end{array}$
$\begin{array}{lllll}\text { H } & 0.540 & 0.974 & -2.429\end{array}$
H $2.141 \quad 1.195 \quad-3.147$
$\begin{array}{llll}H & 1.060 & 2.575 & -2.975\end{array}$
$\begin{array}{llll}C & -0.992 & 0.396 & -0.063\end{array}$
H $-1.354 \quad 1.419-0.176$
$\begin{array}{lllll}\text { O } & -3.984 & 0.951 & -0.181\end{array}$

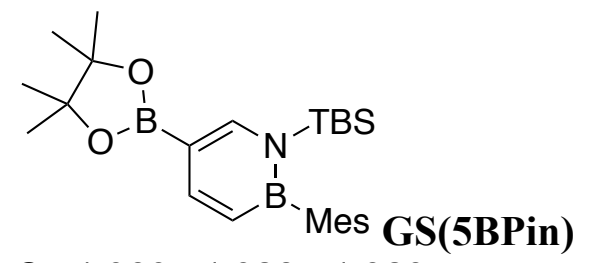

C $1.9691 .928-1.320$

C $1.533 \quad 1.223 \quad-0.189$

C $2.053 \quad 1.586 \quad 1.075$

C $1.567 \quad 0.886 \quad 2.323$

H $1.987 \quad 1.344 \quad 3.222$

$\begin{array}{llll}H & 0.475 & 0.924 & 2.398\end{array}$

$\begin{array}{llll}\text { H } & 1.845 & -0.174 & 2.325\end{array}$

C $3.012 \quad 2.587 \quad 1.177$

H $3.404 \quad 2.854 \quad 2.157$

C 3.4853 .2620 .046

C 4.5394 .3320 .180

H $4.225 \quad 5.106 \quad 0.887$

H $\quad \begin{array}{llll}5.478 & 3.913 & 0.556\end{array}$

H $4.743 \quad 4.810 \quad-0.781$

$\begin{array}{lllll}\text { B } & 0.414 & 0.107 & -0.249\end{array}$

$\begin{array}{llll}\text { N } & 0.723 & -1.311 & -0.114\end{array}$

$\begin{array}{llll}C & -0.312 & -2.206 & -0.012\end{array}$

$\begin{array}{llll}\text { H } & -0.041 & -3.252 & 0.123\end{array}$

$\begin{array}{llll}C & -1.644 & -1.882 & -0.070\end{array}$

$\begin{array}{llll}\text { B } & -2.688 & -3.014 & 0.047\end{array}$

$\begin{array}{llll}\text { O } & -2.359 & -4.327 & 0.294\end{array}$

$\begin{array}{llll}C & -3.554 & -5.108 & 0.058\end{array}$ 

C $-3.572 \quad-6.290 \quad 1.013$
$\begin{array}{llll}\mathrm{H} & -3.457 & -5.967 & 2.048\end{array}$
$\begin{array}{llll}H & -4.513 & -6.841 & 0.917\end{array}$
H $-2.752 \quad-6.970 \quad 0.769$
$\begin{array}{llll}\text { C } & -4.685 & -4.057 & 0.297\end{array}$
$\begin{array}{llll}C & -5.056 & -3.912 & 1.771\end{array}$
$\begin{array}{llll}\mathrm{H} & -4.161 & -3.782 & 2.387\end{array}$
$\begin{array}{llll}H & -5.606 & -4.786 & 2.131\end{array}$
$\begin{array}{llll}H & -5.688 & -3.030 & 1.890\end{array}$
$\begin{array}{llll}\text { C } & -5.931 & -4.259 & -0.549\end{array}$
$\begin{array}{llll}\mathrm{H} & -6.667 & -3.487 & -0.309\end{array}$
$\begin{array}{llll}H & -6.378 & -5.236 & -0.341\end{array}$
H $-5.701-4.198-1.614$
C $-3.491-5.590-1.389$
$\begin{array}{llll}\mathrm{H} & -2.562 & -6.147 & -1.533\end{array}$
H $-4.332-6.245-1.629$
H $-3.495-4.744-2.083$
$\begin{array}{lllll}O & -4.041 & -2.822 & -0.098\end{array}$
$\begin{array}{llll}C & -2.009 & -0.510 & -0.257\end{array}$
$\begin{array}{llll}\mathrm{H} & -3.072 & -0.280 & -0.315\end{array}$
Si 2.358 -2.106 $\quad-0.214$
$\begin{array}{llll}\text { C } 2.420 & -3.398 & 1.151\end{array}$
$\begin{array}{llll}\text { H } & 2.108 & -2.959 & 2.105\end{array}$
H $3.449-3.7501 .269$
H $1.791-4.2720 .962$
$\begin{array}{llll}\text { C } & 3.795 & -0.931 & 0.037\end{array}$
H $3.760-0.441 \quad 1.012$
H $4.709-1.534 \quad 0.002$
$\begin{array}{llll}\text { H } & 3.862 & -0.152 & -0.726\end{array}$
C $2.489-2.914-1.934$
$\begin{array}{llll}\text { C } & 3.842 & -3.639 & -2.022\end{array}$
H $3.932-4.426-1.265$
H $4.685-2.950-1.900$
H $3.947-4.113-3.006$
$\begin{array}{llll}\text { C } & 1.371 & -3.935 & -2.194\end{array}$
$\begin{array}{llll}\text { H } & 1.352 & -4.729 & -1.438\end{array}$
$\begin{array}{llll}\text { H } & 0.382 & -3.464 & -2.219\end{array}$
H $1.531-4.414-3.168$
$\begin{array}{llll}\text { C } & 2.422 & -1.827 & -3.014\end{array}$
$\begin{array}{llll}\text { H } & 3.223 & -1.088 & -2.898\end{array}$
H $1.464-1.295$-2.983
$\begin{array}{llll}\text { H } & 2.524 & -2.274 & -4.011\end{array}$
$\begin{array}{llll}C & -1.063 & 0.470 & -0.347\end{array}$
$\begin{array}{llll}\mathrm{H} & -1.391 & 1.501 & -0.474\end{array}$
C $2.945 \quad 2.926-1.190$
H $3.278 \quad 3.459 \quad-2.079$
C $1.4191 .643-2.699$
$\begin{array}{llll}\text { H } & 0.518 & 1.026 & -2.658\end{array}$
$\begin{array}{llll}H & 1.168 & 2.575 & -3.214\end{array}$
H $2.155 \quad 1.120-3.319$ 


\section{NMO}

$\begin{array}{rrrr}\text { H } & 1.873 & 2.023 & -0.165 \\ \text { H } & -0.046 & 1.214 & -1.508 \\ \text { C } & -0.097 & 1.213 & -0.418 \\ \text { C } & 1.297 & 1.164 & 0.189 \\ \text { H } & -0.670 & 2.081 & -0.086 \\ \text { O } & 1.986 & 0.000 & -0.225 \\ \text { H } & 1.257 & 1.214 & 1.288 \\ \text { H } & -0.046 & -1.214 & -1.508 \\ \text { O } & -2.044 & 0.000 & -0.860 \\ \text { N } & -0.919 & 0.000 & -0.093 \\ \text { C } & 1.298 & -1.164 & 0.189 \\ \text { C } & -0.097 & -1.213 & -0.418 \\ \text { H } & 1.873 & -2.023 & -0.165 \\ \text { C } & -1.329 & 0.000 & 1.339 \\ \text { H } & -1.940 & 0.890 & 1.484 \\ \text { H } & 1.257 & -1.214 & 1.288 \\ \text { H } & -0.670 & -2.081 & -0.086 \\ \text { H } & -0.477 & 0.000 & 2.020 \\ \text { H } & -1.940 & -0.890 & 1.484\end{array}$<smiles></smiles>

$\begin{array}{llll}\text { H } & 1.809 & -3.807 & 0.381\end{array}$

TS1(4Bpin)

H $3.390-3.075 \quad 0.670$

$\begin{array}{llll}\text { C } 2.367 & -2.867 & 0.341\end{array}$

H $4.020-4.589-1.466$

H $1.918-2.178 \quad 1.064$

H $1.459-4.960-1.747$

C $4.004-4.103-2.448$

H $-0.015-3.102-0.809$

C $1.547-4.453-2.715$

H $4.174-4.880-3.204$

H $1.764-5.222 \quad-3.469$

Si $2.405-2.079-1.370$

$\begin{array}{llll}\text { H } & 4.849 & -3.408 & -2.498\end{array}$

C $2.664-3.400-2.716$

$\begin{array}{llll}C & -0.278 & -2.133 & -1.223\end{array}$

H $0.574-4.015 \quad-2.961$

H $-2.342-2.471-0.955$

H $4.740-1.430-1.183$

C $-1.588-1.778-1.314$

C $3.828-0.860-1.395$

H $-1.631-0.782 \quad 1.935$ 

H $3.724-0.104-0.613$
$\begin{array}{llll}\mathrm{N} & 0.774 & -1.319 & -1.610\end{array}$
$\begin{array}{llll}\mathrm{H} & -0.934 & 0.040 & 0.515\end{array}$
$\begin{array}{llll}\text { H } & 1.739 & 0.737 & 0.290\end{array}$
$\begin{array}{llll}H & -7.039 & -1.990 & -1.733\end{array}$
$\begin{array}{llll}\text { C } & -1.396 & 0.184 & 1.490\end{array}$
$\begin{array}{llll}\text { C } & 2.702 & -2.727 & -4.092\end{array}$
H $-5.794-2.008$-3.821
$\begin{array}{llll}\text { O } & -4.511 & -1.051 & -1.790\end{array}$
$\begin{array}{llll}\text { C } & -6.907 & -0.915 & -1.585\end{array}$
$\begin{array}{llll}C & 1.612 & 1.746 & -0.121\end{array}$
H $2.904-3.470-4.875$
$\begin{array}{llll}\mathrm{H} & -0.744 & 0.765 & 2.144\end{array}$
$\begin{array}{llll}O & -3.506 & 0.046 & 0.515\end{array}$
$\begin{array}{llll}\text { H } & 3.952 & -0.341 & -2.347\end{array}$
C $-1.980-0.498-1.819$
$\begin{array}{llll}\mathrm{H} & -6.789 & -0.724 & -0.517\end{array}$
$\begin{array}{llll}H & 2.106 & 2.453 & 0.550\end{array}$
$\begin{array}{llll}\text { B } & 0.474 & -0.010 & -2.165\end{array}$
H $3.482-1.959-4.152$
H $0.535 \quad 1.954 \quad-0.106$
$\begin{array}{llll}C & -5.765 & -0.915 & -3.810\end{array}$
$\begin{array}{llll}\text { H } & 1.743 & -2.252 & -4.322\end{array}$
B $-3.503-0.108-1.806$
$\begin{array}{llll}\text { C } & -5.685 & -0.443 & -2.359\end{array}$
$\begin{array}{llll}\mathrm{N} & -2.701 & 0.871 & 1.262\end{array}$
$\begin{array}{llll}\mathrm{H} & -3.445 & 0.202 & 3.103\end{array}$
$\begin{array}{llll}\mathrm{H} & -7.810 & -0.407 & -1.940\end{array}$
$\begin{array}{llll}C & -1.001 & 0.360 & -2.262\end{array}$
C $2.145 \quad 1.851 \quad-1.531$
H $-4.885-0.591 \quad-4.373$
$\begin{array}{llll}\mathrm{H} & -1.940 & 1.965 & -0.357\end{array}$
$\begin{array}{lrrr}C & 1.612 & 1.026 & -2.546\end{array}$
H $-6.662-0.538-4.309$
$\begin{array}{llll}C & -3.395 & 1.149 & 2.563\end{array}$
$\begin{array}{llll}C & -2.508 & 2.180 & 0.551\end{array}$
$\begin{array}{llll}\text { C } & 3.156 & 2.762 & -1.821\end{array}$
H $3.5643 .380 \quad-1.023$
$\begin{array}{llll}H & -1.722 & 1.931 & 3.721\end{array}$
$\begin{array}{llll}\mathrm{H} & -5.464 & 1.129 & -0.074\end{array}$
$\begin{array}{llll}\mathrm{H} & -0.820 & 2.947 & 1.700\end{array}$
C $-5.387 \quad 1.093 \quad-2.232$
$\begin{array}{lllll}\mathrm{O} & -3.946 & 1.133 & -2.221\end{array}$
$\begin{array}{llll}C & -2.715 & 2.247 & 3.369\end{array}$
$\begin{array}{llll}\mathrm{H} & -4.402 & 1.452 & 2.274\end{array}$
$\begin{array}{llll}\mathrm{H} & -1.319 & 1.331 & -2.648\end{array}$
C $2.075 \quad 1.201-3.858$
$\begin{array}{llll}C & -5.883 & 1.689 & -0.915\end{array}$
$\begin{array}{llll}C & -1.852 & 3.226 & 1.439\end{array}$
$\begin{array}{llll}\text { H } & 0.654 & -0.200 & -4.711\end{array}$
$\begin{array}{llll}\mathrm{H} & -3.515 & 2.491 & 0.271\end{array}$
H $-6.9751 .689-0.864$
C $3.662 \quad 2.898 \quad-3.117$
$\begin{array}{llll}C & 1.480 & 0.445 & -5.024\end{array}$
$\begin{array}{llll}\text { H } & 2.228 & -0.179 & -5.523\end{array}$ 


$\begin{array}{rrrr}\text { C } & 3.102 & 2.117 & -4.123 \\ \text { H } & -3.326 & 2.472 & 4.246 \\ \text { H } & 5.460 & 3.973 & -2.595 \\ \text { O } & -2.613 & 3.438 & 2.614 \\ \text { C } & -5.887 & 1.930 & -3.401 \\ H & -5.439 & 1.613 & -4.343 \\ \text { H } & -1.816 & 4.176 & 0.901 \\ \text { C } & 4.745 & 3.902 & -3.419 \\ H & -6.976 & 1.858 & -3.486 \\ H & -5.540 & 2.727 & -0.852 \\ H & 3.464 & 2.225 & -5.144 \\ \text { H } & 5.291 & 3.635 & -4.327 \\ H & 1.091 & 1.144 & -5.771 \\ \text { H } & -5.628 & 2.979 & -3.233 \\ \text { H } & 4.321 & 4.901 & -3.570\end{array}$<smiles>C[B-]1(C2=CN(C(C)(C)C)B(N3CCOCC3)C=C2)OC(C)(C)C(C)(C)O1</smiles>

$\begin{array}{llll}\text { H } & -5.301 & -2.839 & 0.525\end{array}$

H $-4.519-1.667 \quad 1.610$

$\begin{array}{llll}\mathrm{H} & -3.931 & -3.337 & 1.533\end{array}$

$\begin{array}{llll}\text { C } & -4.343 & -2.513 & 0.944\end{array}$

$\begin{array}{llll}\mathrm{H} & -5.674 & -0.350 & -0.083\end{array}$

$\mathrm{H}-2.868 \quad 2.7012 .496$

H $-0.978 \quad 1.350 \quad 3.222$

$\begin{array}{llll}\text { C } & -4.690 & 0.057 & -0.336\end{array}$

$\begin{array}{llll}\mathrm{H} & -4.174 & 0.383 & 0.569\end{array}$

C $-3.371-2.145-0.166$

$\begin{array}{llll}C & -2.273 & 3.134 & 1.691\end{array}$

$\begin{array}{llll}\text { C } & -0.367 & 1.771 & 2.424\end{array}$

$\begin{array}{llll}\mathrm{H} & -5.468 & -1.974 & -2.107\end{array}$

$\begin{array}{llll}H & -3.819 & 4.289 & 0.758\end{array}$

$\begin{array}{llll}\text { H } & -3.860 & -3.888 & -1.392\end{array}$

$\begin{array}{lllll}\mathrm{O} & -1.977 & 0.944 & 0.954\end{array}$

H $-1.722 \quad 4.0092 .049$

H $-2.483-4.095-0.290$

$\begin{array}{llll}\text { C } & -3.134 & 3.483 & 0.488\end{array}$

$\begin{array}{llll}C & -2.985 & -3.393 & -0.962\end{array}$

$\begin{array}{llll}\mathrm{H} & -3.706 & 2.598 & 0.191\end{array}$

$\begin{array}{llll}H & -4.835 & 0.926 & -0.986\end{array}$

C $-3.844-0.971-1.085$

$\begin{array}{llll}\text { O } & -2.164 & -1.627 & 0.415\end{array}$

H $0.149 \quad 2.674 \quad 2.756$

$\begin{array}{llll}\mathrm{N} & -1.277 & 2.079 & 1.292\end{array}$

$\begin{array}{llll}C & -4.562 & -1.411 & -2.353\end{array}$

$\begin{array}{llll}\mathrm{H} & 0.345 & 1.028 & 2.063\end{array}$

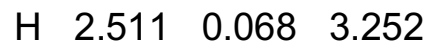

H $-4.855-0.530-2.931$

$\begin{array}{llll}\text { B } & -1.674 & -0.653 & -0.455\end{array}$ 

C $2.328-1.000 \quad 3.096$
H $-2.291-3.137-1.769$
H $2.834-1.538 \quad 3.904$
H $-3.921 \quad-2.034 \quad-2.979$
$\begin{array}{llll}\text { O } & -2.597 & -0.355 & -1.454\end{array}$
H $\quad 0.382 \quad-1.307 \quad 1.133$
$\begin{array}{lllll}O & -2.343 & 3.948 & -0.590\end{array}$
$\begin{array}{lrrr}\text { H } & 5.311 & -1.720 & 2.287\end{array}$
$\begin{array}{llll}\text { C } & -0.476 & 2.625 & 0.140\end{array}$
H $1.255-1.182 \quad 3.202$
H $5.083-0.126 \quad 1.552$
C $4.870-1.191 \quad 1.435$
$\begin{array}{llll}\text { C } & -1.421 & 2.950 & -0.999\end{array}$
$\begin{array}{llll}C & 0.760 & -0.853 & 0.219\end{array}$
$\begin{array}{llll}\mathrm{H} & -1.946 & 2.037 & -1.312\end{array}$
H $\quad 0.029 \quad 3.530 \quad 0.490$
$\begin{array}{llll}\text { C } & -0.143 & -0.478 & -0.737\end{array}$
$\begin{array}{llll}H & 0.256 & 1.867 & -0.133\end{array}$
Si $3.033-1.552 \quad 1.442$
H $5.370-1.5290 .522$
$\begin{array}{llll}N & 2.125 & -0.675 & 0.139\end{array}$
$\begin{array}{llll}\mathrm{H} & -0.853 & 3.356 & -1.840\end{array}$
$\begin{array}{llll}C & 0.353 & 0.102 & -1.947\end{array}$
$\begin{array}{llll}\text { H } & -0.380 & 0.349 & -2.716\end{array}$
$\begin{array}{llll}\text { C } & 2.758 & -3.421 & 1.170\end{array}$
$\begin{array}{llll}\text { B } & 2.673 & 0.020 & -1.015\end{array}$
$\begin{array}{lll}\text { C } 3.636 & 2.274 & 0.621\end{array}$
C $1.683 \quad 0.371-2.120$
C $4.599 \quad 1.597 \quad-0.327$
$\begin{array}{llll}\text { C } & 4.183 & 0.490 & -1.092\end{array}$
$\begin{array}{llll}\text { H } & 2.002 & 0.844 & -3.048\end{array}$
$\begin{array}{llll}H & 6.217 & 2.920 & 0.174\end{array}$
$\begin{array}{llll}\text { C } 5.910 & 2.066 & -0.427\end{array}$
$\begin{array}{llll}\text { C } & 5.103 & -0.102 & -1.975\end{array}$
C $6.835 \quad 1.464-1.279$
C $4.679-1.257-2.850$
$\begin{array}{llll}\text { C } & 6.412 & 0.378 & -2.048\end{array}$
C $8.242 \quad 1.992 \quad-1.398$
$\begin{array}{llll}\text { H } & 7.119 & -0.101 & -2.724\end{array}$
H $3.362 \quad 1.6131 .454$
H $2.704 \quad 2.541 \quad 0.113$
H $4.0693 .183 \quad 1.044$
$\begin{array}{llll}H & 8.326 & 2.697 & -2.232\end{array}$
H $8.954 \quad 1.184 \quad-1.581$
$\begin{array}{llll}\text { H } & 8.546 & 2.519 & -0.490\end{array}$
$\begin{array}{llll}\text { H } & 4.189 & -2.042 & -2.266\end{array}$
H $5.533-1.699-3.369$
$\begin{array}{llll}\text { H } & 3.956 & -0.929 & -3.605\end{array}$
C $1.317 \quad-3.865 \quad 1.467$
C $3.700-4.183 \quad 2.119$
$\begin{array}{llll}C & 3.103 & -3.792 & -0.278\end{array}$
$\begin{array}{llll}\text { H } & 2.449 & -3.277 & -0.992\end{array}$
H $2.981-4.872 \quad-0.431$
$\begin{array}{llll}\text { H } & 4.142 & -3.541 & -0.522\end{array}$
$\begin{array}{llll}\text { H } & 3.514 & -3.932 & 3.170\end{array}$ 
$\begin{array}{llll}H & 4.753 & -3.979 & 1.901 \\ H & 3.543 & -5.263 & 2.008 \\ H & 0.998 & -3.578 & 2.476 \\ H & 1.251 & -4.959 & 1.402 \\ H & 0.598 & -3.454 & 0.753\end{array}$

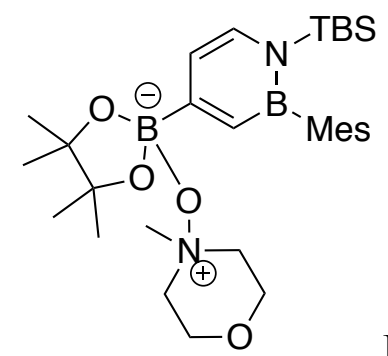

$\begin{array}{llll}C & 1.095 & -0.504 & -0.172\end{array}$

INT(4Bpin)

$\begin{array}{llll}\text { C } & 0.167 & 0.284 & 0.475\end{array}$

$\begin{array}{llll}\text { H } & 0.541 & 0.997 & 1.214\end{array}$

$\begin{array}{llll}\text { B } & -1.317 & 0.183 & 0.170\end{array}$

$\begin{array}{llll}\text { C } & -2.370 & 1.190 & 0.797\end{array}$

C $-2.835 \quad 1.090 \quad 2.118$

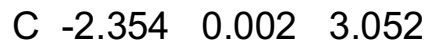

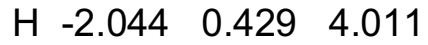

$\begin{array}{llll}\mathrm{H} & -1.503 & -0.540 & 2.633\end{array}$

$\begin{array}{llll}\mathrm{H} & -3.146 & -0.724 & 3.263\end{array}$

$\begin{array}{llll}C & -3.762 & 2.018 & 2.607\end{array}$

H $-4.121 \quad 1.919 \quad 3.631$

$\begin{array}{llll}C & -4.224 & 3.073 & 1.825\end{array}$

$\begin{array}{llll}C & -5.250 & 4.043 & 2.355\end{array}$

$\begin{array}{llll}\mathrm{H} & -5.145 & 4.180 & 3.434\end{array}$

$\begin{array}{llll}H & -6.266 & 3.678 & 2.168\end{array}$

H $\quad-5.158 \quad 5.019 \quad 1.873$

$\begin{array}{llll}\text { C } & -3.711 & 3.204 & 0.532\end{array}$

H $-4.030 \quad 4.043-0.084$

$\begin{array}{llll}C & -2.800 & 2.285 & 0.017\end{array}$

C $-2.278 \quad 2.466-1.390$

H -1.185 $2.397-1.416$

H -2.572 $3.435 \quad-1.801$

H $-2.653 \quad 1.686-2.064$

$\begin{array}{llll}\mathrm{N} & -1.710 & -0.818 & -0.814\end{array}$

Si $-3.388-1.363-1.225$

$\begin{array}{llll}C & -3.704 & -3.010 & -0.319\end{array}$

$\begin{array}{llll}C & -3.593 & -2.784 & 1.193\end{array}$

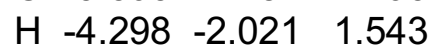

$\begin{array}{llll}\mathrm{H} & -3.813 & -3.714 & 1.734\end{array}$

H $-2.583 \quad-2.466 \quad 1.472$

$\begin{array}{llll}\text { C } & -5.125 & -3.486 & -0.662\end{array}$

H $-5.887-2.783-0.312$

$\begin{array}{llll}H & -5.321 & -4.451 & -0.178\end{array}$

H $-5.260 \quad-3.626-1.741$

$\begin{array}{llll}C & -2.708 & -4.104 & -0.729\end{array}$

$\begin{array}{llll}\mathrm{H} & -2.965 & -5.044 & -0.224\end{array}$

$\begin{array}{llll}\mathrm{H} & -1.682 & -3.849 & -0.446\end{array}$

$\begin{array}{llll}H & -2.728 & -4.296 & -1.808\end{array}$ 

$\begin{array}{llll}C & -4.735 & -0.143 & -0.764\end{array}$
$\begin{array}{llll}\mathrm{H} & -4.806 & 0.047 & 0.309\end{array}$
$\begin{array}{llll}\text { H } & -5.683 & -0.570 & -1.108\end{array}$
$\begin{array}{llll}H & -4.605 & 0.819 & -1.263\end{array}$
C $-3.455-1.595-3.093$
H $-4.497-1.719-3.404$
$\begin{array}{llll}\mathrm{H} & -3.061 & -0.707 & -3.598\end{array}$
$\begin{array}{llll}\text { H } & -2.898 & -2.464 & -3.454\end{array}$
$\begin{array}{llll}\text { C } & -0.720 & -1.563 & -1.422\end{array}$
$\begin{array}{llll}\mathrm{H} & -1.046 & -2.289 & -2.164\end{array}$
$\begin{array}{llll}\text { B } & 2.684 & -0.413 & 0.149\end{array}$
$\begin{array}{llll}\text { O } & 2.965 & -0.448 & 1.565\end{array}$
C $4.134-1.238 \quad 1.756$
C $4.077-1.875 \quad 3.138$
H $4.172-1.102 \quad 3.906$
H $3.127 \quad-2.392 \quad 3.290$
H $4.894 \quad-2.592 \quad 3.274$
C $5.373-0.343 \quad 1.650$
$\begin{array}{llll}H & 5.261 & 0.489 & 2.351\end{array}$
$\begin{array}{llll}\text { H } & 6.290 & -0.888 & 1.899\end{array}$
$\begin{array}{llll}\text { H } & 5.463 & 0.070 & 0.642\end{array}$
$\begin{array}{llll}\text { C } & 4.042 & -2.252 & 0.574\end{array}$
$\begin{array}{llll}C & 3.064 & -3.392 & 0.873\end{array}$
H $3.462 \quad-4.093 \quad 1.614$
H $2.114 \quad-2.991 \quad 1.238$
H $2.872 \quad-3.940 \quad-0.054$
$\begin{array}{llll}\text { C } & 5.382 & -2.821 & 0.124\end{array}$
$\begin{array}{llll}\text { H } & 5.876 & -3.354 & 0.943\end{array}$
$\begin{array}{llll}\text { H } & 6.046 & -2.028 & -0.228\end{array}$
$\begin{array}{llll}\text { H } & 5.227 & -3.528 & -0.697\end{array}$
$\begin{array}{lllll}\text { O } & 3.248 & 1.007 & -0.274\end{array}$
$\begin{array}{llll}\mathrm{N} & 3.204 & 1.464 & -1.591\end{array}$
C $4.3202 .469-1.688$
$\begin{array}{llll}\text { C } 4.051 & 3.628 & -0.742\end{array}$
$\begin{array}{llll}\text { H } & 4.059 & 3.270 & 0.294\end{array}$
H $4.834 \quad 4.377 \quad-0.870$
$\begin{array}{llll}\text { O } & 2.819 & 4.248 & -1.052\end{array}$
$\begin{array}{llll}\text { C } & 1.746 & 3.337 & -0.905\end{array}$
$\begin{array}{llll}H & 1.684 & 2.964 & 0.125\end{array}$
$\begin{array}{llll}H & 0.827 & 3.877 & -1.145\end{array}$
H $5.236 \quad 1.935 \quad-1.433$
H $4.353 \quad 2.814 \quad-2.724$
C $1.9002 .170-1.863$
H $1.108 \quad 1.431 \quad-1.740$
H $1.943 \quad 2.519 \quad-2.898$
$\begin{array}{llll}\text { C } 3.421 & 0.375 & -2.585\end{array}$
H $4.330-0.152 \quad-2.305$
$\begin{array}{llll}\text { H } & 2.574 & -0.306 & -2.534\end{array}$
$\begin{array}{llll}\text { H } & 3.495 & 0.833 & -3.572\end{array}$
$\begin{array}{lllll}\text { O } & 3.493 & -1.458 & -0.471\end{array}$
$\begin{array}{llll}\text { C } & 0.608 & -1.439 & -1.140\end{array}$
$\begin{array}{llll}\text { H } & 1.311 & -2.086 & -1.657\end{array}$ 


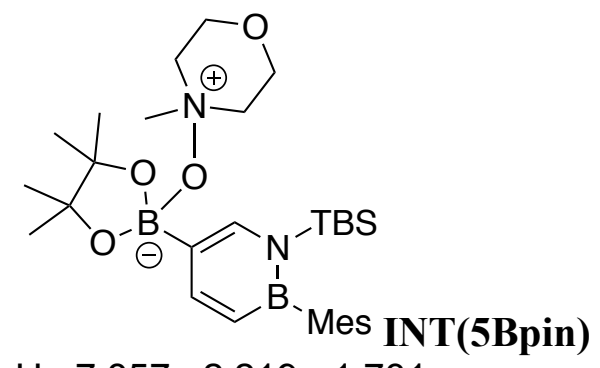

$\begin{array}{llll}\text { H } & 7.857 & 2.219 & -1.731\end{array}$

$\begin{array}{llll}H & 8.177 & 0.667 & -0.961\end{array}$

H $5.875 \quad 2.317 \quad-0.056$

C $7.6291 .151-1.776$

H $3.842 \quad 2.856 \quad 0.991$

C $5.388 \quad 1.584-0.698$

$\begin{array}{llll}H & 8.019 & 0.757 & -2.718\end{array}$

C $3.2372 .084 \quad 0.509$

$\begin{array}{llll}\text { C } & 6.147 & 0.902 & -1.652\end{array}$

H $2.886 \quad 1.397 \quad 1.288$

$\begin{array}{llll}C & 4.022 & 1.346 & -0.551\end{array}$

$\begin{array}{llll}H & 2.346 & 2.558 & 0.082\end{array}$

$\begin{array}{llll}\text { H } & 2.058 & -0.370 & 2.738\end{array}$

$\begin{array}{llll}\text { H } & 4.339 & -0.943 & 0.757\end{array}$

$\begin{array}{llll}\text { C } & 5.497 & -0.018 & -2.473\end{array}$

H $4.329-2.616 \quad 1.344$

H $2.029-2.053 \quad 3.282$

C $1.626-1.356 \quad 2.540$

C $3.912-1.934 \quad 0.596$

H $-1.132 \quad 2.5902 .069$

$\begin{array}{llll}\text { H } & -0.590 & 3.964 & 0.073\end{array}$

$\begin{array}{llll}\text { C } & 3.376 & 0.401 & -1.368\end{array}$

H 6.068 -0.549 $\quad-3.233$

$\begin{array}{llll}\text { O } & -2.522 & 4.320 & 0.596\end{array}$

$\begin{array}{llll}\text { C } & 4.129 & -0.269 & -2.346\end{array}$

$\begin{array}{llll}C & -1.537 & 3.422 & 0.118\end{array}$

C $-1.385 \quad 2.245 \quad 1.063$

H $0.544 \quad-1.293 \quad 2.697$

H $4.229-2.277 \quad-0.393$

Si $\begin{array}{llll} & 2.050 & -1.938 & 0.803\end{array}$

H $-3.4692 .846 \quad 2.630$

$\begin{array}{llll}\mathrm{H} & -0.644 & 1.528 & 0.711\end{array}$

$\begin{array}{llll}\text { B } & 1.814 & 0.165 & -1.242\end{array}$

$\begin{array}{llll}H & -2.359 & 0.865 & 3.152\end{array}$

H $2.767 \quad-4.5592 .010$

$\begin{array}{llll}\mathrm{N} & 1.226 & -0.807 & -0.344\end{array}$

C $-3.783 \quad 3.685 \quad 0.660$

$\begin{array}{llll}C & -3.746 & 2.513 & 1.627\end{array}$

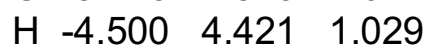

C $0.836 \quad 0.994 \quad-2.072$

$\begin{array}{llll}\text { C } & -2.599 & 0.420 & 2.186\end{array}$

H $1.183 \quad 1.732-2.795$

H $-1.792 \quad 3.062-0.886$

$\begin{array}{llll}\mathrm{N} & -2.704 & 1.518 & 1.185\end{array}$

C $3.458-1.276-3.250$

$\begin{array}{llll}C & 2.472 & -4.704 & 0.964\end{array}$

$\begin{array}{llll}\text { H } & 3.369 & -4.617 & 0.344\end{array}$ 

$\begin{array}{llll}\mathrm{H} & -1.816 & -0.261 & 1.858\end{array}$
$\begin{array}{llll}\text { C } & -0.155 & -0.895 & -0.273\end{array}$
H $\quad 0.327 \quad-3.884 \quad 2.452$
$\begin{array}{llll}\text { C } & 1.373 & -3.714 & 0.533\end{array}$
H $\quad 4.161-1.701-3.970$
$\begin{array}{llll}\text { C } & -0.509 & 0.806 & -1.917\end{array}$
$\begin{array}{llll}\text { H } & 3.026 & -2.099 & -2.668\end{array}$
H $-4.693 \quad 1.973 \quad 1.650$
$\begin{array}{llll}\mathrm{H} & -4.098 & 3.340 & -0.331\end{array}$
$\begin{array}{llll}\text { H } & -0.556 & -1.629 & 0.422\end{array}$
$\begin{array}{llll}\mathrm{H} & -3.552 & -0.104 & 2.202\end{array}$
$\begin{array}{llll}\text { H } & 2.631 & -0.817 & -3.802\end{array}$
$\begin{array}{llll}\text { C }-1.042 & -0.141 & -0.983\end{array}$
$\begin{array}{llll}\text { C } & 0.129 & -4.023 & 1.384\end{array}$
$\begin{array}{lllll}\text { O } & -3.160 & 1.060 & -0.051\end{array}$
$\begin{array}{llll}\text { H } & 2.099 & -5.732 & 0.868\end{array}$
$\begin{array}{llll}\mathrm{H} & -1.227 & 1.382 & -2.506\end{array}$
$\begin{array}{llll}\text { H } & -0.741 & -3.412 & 1.122\end{array}$
H $1.936-3.804-1.580$
C $1.053-3.955-0.949$
$\begin{array}{llll}\text { B } & -2.620 & -0.285 & -0.715\end{array}$
$\begin{array}{llll}\text { H } & -0.160 & -5.072 & 1.236\end{array}$
$\begin{array}{llll}\text { O } & -2.987 & -1.431 & 0.114\end{array}$
H $0.261-3.291 \quad-1.310$
H $\quad 0.718-4.991 \quad-1.097$
$\begin{array}{llll}\text { O } & -3.415 & -0.362 & -1.919\end{array}$
$\begin{array}{llll}\mathrm{H} & -5.401 & -0.214 & -0.067\end{array}$
$\begin{array}{llll}\text { C } & -3.774 & -2.301 & -0.693\end{array}$
$\begin{array}{llll}\mathrm{H} & -2.241 & -3.813 & -0.677\end{array}$
$\begin{array}{llll}\mathrm{H} & -2.157 & -2.720 & -2.073\end{array}$
C $-2.839-3.272-1.418$
$\begin{array}{llll}\mathrm{H} & -4.175 & -3.735 & 0.860\end{array}$
$\begin{array}{llll}\mathrm{H} & -5.332 & -2.394 & 0.813\end{array}$
$\begin{array}{llll}C & -4.454 & -1.308 & -1.688\end{array}$
C $-5.658-0.600-1.056$
$\begin{array}{llll}C & -4.739 & -3.072 & 0.197\end{array}$
$\begin{array}{llll}H & -5.937 & 0.246 & -1.691\end{array}$
$\begin{array}{llll}\text { H } & -6.519 & -1.270 & -0.969\end{array}$
H $-3.391-4.005-2.015$
C $-4.861-1.924-3.020$
$\begin{array}{llll}\mathrm{H} & -5.418 & -3.688 & -0.403\end{array}$
$\begin{array}{llll}\mathrm{H} & -3.988 & -2.297 & -3.559\end{array}$
H $-5.350-1.168-3.641$
$\begin{array}{llll}H & -5.565 & -2.750 & -2.872\end{array}$

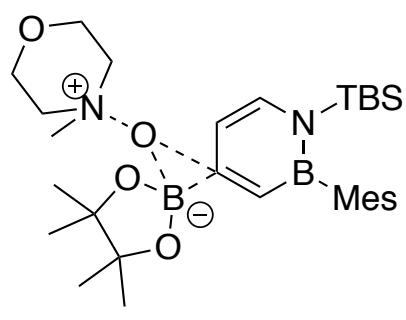

$\begin{array}{llll}\text { H } & -2.412 & -4.837 & 2.562\end{array}$

TS2(4Bpin) 

H -3.668 -3.658 2.980
$\begin{array}{llll}C & -2.828 & -3.858 & 2.306\end{array}$
H $7.383 \quad-1.077 \quad 0.889$
H $4.503-1.921 \quad 2.720$
$\begin{array}{llll}\text { H } & 2.060 & -2.393 \quad 3.036\end{array}$
$\begin{array}{llll}\text { H } & -5.029 & -5.099 & 1.302\end{array}$
$\begin{array}{llll}H & -3.603 & -5.979 & 0.718\end{array}$
H $-2.055 \quad-3.105 \quad 2.471$
$\begin{array}{llll}C & -4.191 & -5.064 & 0.600\end{array}$
H $0.883-1.650 \quad 1.940$
C $6.743-1.924 \quad 1.155$
C $1.881-1.542 \quad 2.372$
C $4.277-1.699 \quad 1.677$
$\begin{array}{llll}C & -3.286 & -3.858 & 0.854\end{array}$
$\begin{array}{llll}\text { H } & -5.755 & -2.610 & 1.577\end{array}$
$\begin{array}{llll}\text { H } & 7.146 & -2.805 & 0.644\end{array}$
H $6.826-2.0892 .231$
C $5.313-1.669 \quad 0.749$
$\begin{array}{llll}\text { C } & 2.947 & -1.467 & 1.302\end{array}$
$\begin{array}{llll}\mathrm{H} & -3.621 & -1.226 & 2.114\end{array}$
H $1.879-0.6412 .994$
$\begin{array}{llll}\mathrm{H} & -4.597 & -5.039 & -0.417\end{array}$
$\begin{array}{llll}O & -2.143 & -3.970 & 0.002\end{array}$
H $-0.909-7.257-1.696$
$\begin{array}{llll}C & -5.462 & -2.474 & 0.531\end{array}$
$\begin{array}{llll}C & -3.948 & -2.519 & 0.383\end{array}$
$\begin{array}{llll}C & -3.327 & -1.294 & 1.063\end{array}$
H $-3.001-5.845-1.640$
$\begin{array}{llll}H & -5.943 & -3.247 & -0.071\end{array}$
H $-2.234-1.322 \quad 1.004$
$\begin{array}{llll}\text { C } & -0.966 & -6.289 & -2.200\end{array}$
$\begin{array}{llll}\mathrm{H} & 0.441 & -3.127 & -0.391\end{array}$
H $-0.582-5.508-1.530$
C $4.989-1.415-0.588$
$\begin{array}{llll}C & -2.413 & -5.979 & -2.548\end{array}$
$\begin{array}{llll}C & 2.634 & -1.169 & -0.033\end{array}$
$\begin{array}{llll}\text { H } & -5.833 & -1.500 & 0.197\end{array}$
$\begin{array}{llll}\text { B } & -2.319 & -3.091 & -1.113\end{array}$
H $1.552 \quad 1.688 \quad 2.410$
H $-2.843 \quad-6.798-3.144$
$\begin{array}{llll}\text { C } & 0.203 & -2.120 & -0.734\end{array}$
$\begin{array}{llll}\text { H } & -3.672 & -0.394 & 0.544\end{array}$
$\begin{array}{llll}\text { O } & -3.613 & -2.489 & -1.005\end{array}$
$\begin{array}{llll}\text { C } & 3.678 & -1.170 & -0.983\end{array}$
$\begin{array}{llll}C & 0.507 & 1.793 & 2.097\end{array}$
$\begin{array}{llll}\text { H } & 5.783 & -1.417 & -1.334\end{array}$
$\begin{array}{llll}\text { H } & -0.052 & 2.217 & 2.943\end{array}$
$\begin{array}{llll}\text { B } & 1.156 & -0.943 & -0.547\end{array}$
H $3.378 \quad 1.3120 .826$
$\begin{array}{llll}\text { O } & -0.178 & -6.381 & -3.373\end{array}$
$\begin{array}{llll}\mathrm{H} & 0.107 & 0.790 & 1.904\end{array}$
C $-1.019-1.910-1.313$
$\begin{array}{llll}\mathrm{H} & -4.424 & -4.240 & -2.742\end{array}$
$\begin{array}{llll}\mathrm{O} & -1.757 & -3.371 & -2.371\end{array}$
$\begin{array}{llll}\mathrm{N} & -2.487 & -4.740 & -3.333\end{array}$ 


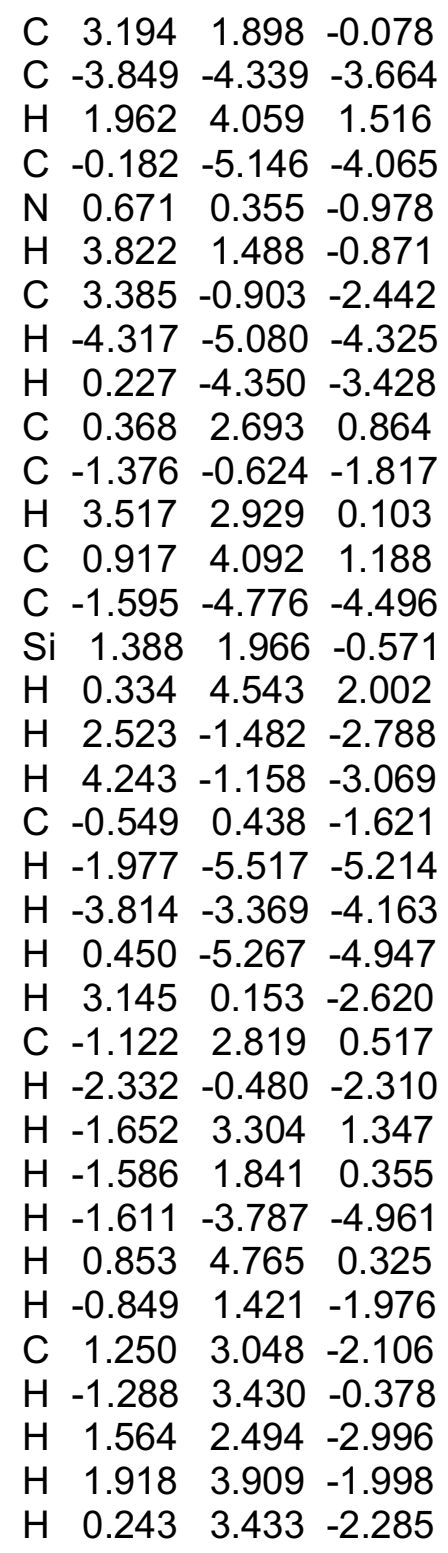

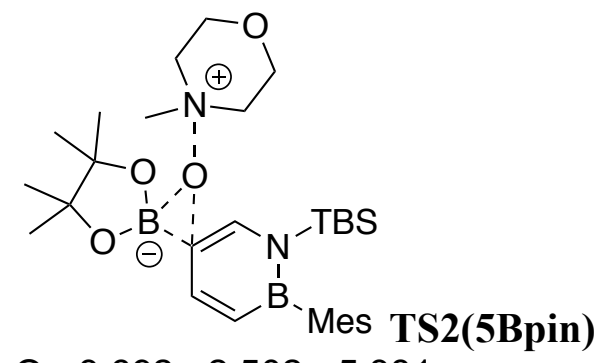

$\begin{array}{llll}\text { O } & -3.692 & -2.502 & -5.381\end{array}$

H $-5.241-3.168$ - 4.254

$\begin{array}{llll}\text { C }-4.472 & -2.395 & -4.203\end{array}$

H $-3.854-2.556-3.309$

H $-2.086-1.670-6.296$

C $-2.655-1.539-5.373$

H $-1.994-1.700-4.511$

C $-5.125-1.027-4.113$

$\begin{array}{llll}C & -3.223 & -0.127 & -5.319\end{array}$

$\begin{array}{llll}\mathrm{H} & -5.817 & -0.884 & -4.957\end{array}$

$\begin{array}{llll}H & -5.673 & -0.933 & -3.175\end{array}$ 


$\begin{array}{lrrr}H & -3.799 & 0.082 & -6.233 \\ \text { N } & -4.104 & 0.025 & -4.157 \\ \text { H } & -2.418 & 0.607 & -5.228 \\ \text { O } & -2.907 & -0.222 & -2.775 \\ \text { C } & -4.641 & 1.371 & -4.014 \\ \text { H } & -5.308 & 1.612 & -4.853 \\ \text { H } & -5.182 & 1.438 & -3.070 \\ \text { H } & -3.809 & 2.077 & -3.991 \\ \text { H } & -2.835 & -0.236 & 1.254 \\ \text { H } & -1.191 & 2.832 & 0.698 \\ \text { H } & -4.070 & 0.315 & 2.410 \\ \text { H } & -0.230 & 3.413 & -1.951 \\ \text { C } & -3.583 & 0.537 & 1.455 \\ \text { H } & -3.065 & 1.496 & 1.545 \\ \text { H } & -4.368 & -1.953 & 1.544 \\ \text { C } & -0.456 & 2.496 & 1.441 \\ \text { H } & 2.195 & -1.001 & 3.267 \\ \text { H } & -0.676 & 3.020 & 2.381 \\ \text { H } & 0.919 & -0.758 & 2.058 \\ \text { C } & 0.769 & 2.993 & -2.101 \\ \text { H } & 0.429 & 4.721 & 0.116 \\ \text { H } & 1.459 & 3.831 & -2.243 \\ \text { C } & 1.795 & -1.362 & 2.315 \\ \text { H } & -0.620 & 1.425 & 1.606 \\ \text { H } & 0.756 & 2.406 & -3.025 \\ \text { H } & 1.438 & -2.386 & 2.475 \\ \text { C } & -5.344 & -1.723 & 1.112 \\ \text { C } & 1.156 & 4.306 & 0.820 \\ \text { H } & 1.014 & 4.811 & 1.784 \\ \text { C } & -4.601 & 0.638 & 0.315 \\ \text { H } & -5.986 & -1.303 & 1.894 \\ \text { H } & -5.794 & -2.658 & 0.766 \\ \text { C } & 0.982 & 2.788 & 0.998 \\ \text { O } & -4.262 & -1.283 & -0.990 \\ \text { B } & -3.424 & -0.211 & -1.453 \\ \text { C } & -1.298 & -1.870 & -1.094 \\ \text { C } & -5.210 & -0.757 & -0.059 \\ \text { C } & -1.746 & -0.527 & -1.277 \\ \text { H } & -2.034 & -2.662 & -1.233 \\ \text { Si } & 1.377 & 1.940 & -0.663 \\ \text { C } & -0.010 & -2.130 & -0.722 \\ \text { O } & -3.918 & 1.009 & -0.882 \\ \text { C } & -0.860 & 0.509 & -1.150 \\ \text { H } & -6.244 & 1.405 & 1.506 \\ \text { B } & 0.969 & -0.978 & -0.539 \\ \text { H } & -5.116 & 2.638 & 0.916 \\ \text { C } & -5.630 & 1.709 & 0.652 \\ \text { C } & 2.844 & -1.339 & 1.226 \\ \text { H } & 0.290 & -3.166 & -0.568 \\ \text { N } & 0.460 & 0.367 & -0.787 \\ \text { H } & 2.160 & 4.564 & 0.462 \\ \text { H } & -1.217 & 1.517 & -1.350 \\ \text { H } & 4.465 & -1.568 & 2.621 \\ \text { C } & 1.957 & 2.311 & 2.085 \\ \text { C } & 4.189 & -1.500 & 1.570\end{array}$



H $\quad \begin{array}{lll}1.690 & 2.761 & 3.050\end{array}$
$\begin{array}{llll}\text { C } & 2.475 & -1.223 & -0.124\end{array}$
H $\quad 1.939 \quad 1.224 \quad 2.207$
$\begin{array}{llll}\text { C } & -6.563 & -0.623 & -0.759\end{array}$
$\begin{array}{llll}C & 3.236 & 1.777 & -0.826\end{array}$
$\begin{array}{llll}\text { H } & -6.286 & 1.912 & -0.197\end{array}$
$\begin{array}{llll}\text { H } & 3.637 & 2.796 & -0.757\end{array}$
$\begin{array}{llll}\text { H } & -6.829 & -1.585 & -1.207\end{array}$
$\begin{array}{llll}\text { H } & -7.352 & -0.339 & -0.057\end{array}$
H $3.540 \quad 1.368$-1.792
H $2.9872 .602 \quad 1.855$
C $5.183-1.590 \quad 0.597$
C $3.467-1.370-1.113$
$\begin{array}{llll}H & -6.524 & 0.133 & -1.550\end{array}$
$\begin{array}{llll}\text { H } & 6.748 & -2.265 & 1.924\end{array}$
$\begin{array}{llll}\text { H } & 3.688 & 1.175 & -0.036\end{array}$
$\begin{array}{llll}\text { C } & 6.635 & -1.722 & 0.982\end{array}$
$\begin{array}{llll}C & 4.799 & -1.546 & -0.745\end{array}$
$\begin{array}{llll}\text { H } & 2.450 & -2.192 & -2.831\end{array}$
H $7.203 \quad-2.249 \quad 0.210$
$\begin{array}{llll}\text { C } & 3.083 & -1.335 & -2.574\end{array}$
$\begin{array}{llll}\text { H } & 2.506 & -0.436 & -2.817\end{array}$
H $7.096-0.737 \quad 1.114$
H 5.558 -1.645 -1.519
H $3.965-1.359$ - 3.219

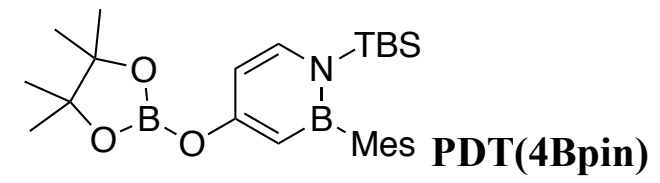

$\begin{array}{llll}C & -1.397 & -2.478 & 0.357\end{array}$

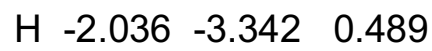

$\begin{array}{llll}C & -0.046 & -2.621 & 0.327\end{array}$

$\begin{array}{llll}\text { H } & 0.379 & -3.616 & 0.432\end{array}$

$\begin{array}{llll}\text { N } & 0.840 & -1.576 & 0.167\end{array}$

$\begin{array}{llll}\text { B } & 0.299 & -0.227 & 0.056\end{array}$

$\begin{array}{llll}\text { C } & -1.210 & -0.070 & 0.070\end{array}$

$\begin{array}{llll}\mathrm{H} & -1.689 & 0.898 & -0.020\end{array}$

C $1.248 \quad 1.041 \quad 0.029$

C $1.798 \quad 1.494 \quad 1.250$

$\begin{array}{llll}C & 1.470 & 0.777 & 2.540\end{array}$

H $1.909 \quad 1.290 \quad 3.400$

$\begin{array}{llll}\mathrm{H} & 0.387 & 0.716 & 2.691\end{array}$

H $1.843 \quad-0.254 \quad 2.536$

C $2.642 \quad 2.599 \quad 1.273$

H $3.0602 .933 \quad 2.222$

C $2.9653 .294 \quad 0.102$

C $3.905 \quad 4.472 \quad 0.147$

H $4.900 \quad 4.166 \quad 0.485$

H $3.548 \quad 5.234 \quad 0.846$

H $4.010 \quad 4.934 \quad-0.837$

C $2.384 \quad 2.872 \quad-1.088$

H $2.594 \quad 3.419 \quad-2.006$

C $1.5191 .770-1.136$ 

$\begin{array}{llll}\text { C } & 0.881 & 1.430 & -2.465\end{array}$
$\begin{array}{llll}\mathrm{H} & 0.163 & 0.612 & -2.374\end{array}$
$\begin{array}{llll}H & 0.348 & 2.299 & -2.863\end{array}$
H $1.6321 .144 \quad-3.208$
Si $2.570-2.094 \quad-0.022$
$\begin{array}{llll}\text { C } & 2.874 & -3.442 & 1.259\end{array}$
H $2.493 \quad-3.133 \quad 2.237$
H $3.953-3.601 \quad 1.357$
H $2.422-4.406 \quad 1.008$
$\begin{array}{llll}\text { C } & 3.826 & -0.736 & 0.282\end{array}$
H $3.751 \quad-0.332 \quad 1.294$
H $4.816-1.193 \quad 0.180$
H $3.751 \quad 0.099 \quad-0.418$
C $2.780-2.774-1.786$
C $4.221-3.283-1.945$
H $4.455-4.079 \quad-1.228$
H $4.955-2.481-1.815$
H $4.360-3.696 \quad-2.953$
$\begin{array}{llll}\text { C } & 1.813 & -3.931 & -2.074\end{array}$
H $1.943-4.763-1.372$
H 0.768 -3.608 $\quad-2.030$
$\begin{array}{llll}\text { H } & 1.994 & -4.324 & -3.083\end{array}$
C $2.519-1.653-2.799$
$\begin{array}{llll}\text { H } & 3.187 & -0.798 & -2.647\end{array}$
H $1.487-1.292 \quad-2.728$
$\begin{array}{llll}\text { H } & 2.676 & -2.019 & -3.822\end{array}$
$\begin{array}{llll}\text { C } & -1.986 & -1.186 & 0.217\end{array}$
$\begin{array}{llll}\text { O } & -3.356 & -1.217 & 0.265\end{array}$
$\begin{array}{llll}\text { B } & -4.227 & -0.170 & 0.160\end{array}$
$\begin{array}{llll}\text { O } & -5.570 & -0.405 & 0.312\end{array}$
$\begin{array}{llll}C & -6.244 & 0.805 & -0.106\end{array}$
$\begin{array}{llll}\text { C } & -7.501 & 0.984 & 0.729\end{array}$
$\begin{array}{llll}\mathrm{H} & -7.276 & 0.954 & 1.797\end{array}$
$\begin{array}{llll}\mathrm{H} & -7.979 & 1.940 & 0.497\end{array}$
$\begin{array}{llll}\mathrm{H} & -8.208 & 0.183 & 0.502\end{array}$
$\begin{array}{llll}C & -5.145 & 1.892 & 0.126\end{array}$
$\begin{array}{llll}C & -5.100 & 2.391 & 1.567\end{array}$
H $\quad-5.082 \quad 1.555 \quad 2.272$
H $-5.963 \quad 3.022 \quad 1.795$
$\begin{array}{llll}H & -4.191 & 2.980 & 1.708\end{array}$
$\begin{array}{llll}\text { C } & -5.187 & 3.060 & -0.843\end{array}$
$\begin{array}{llll}H & -4.382 & 3.760 & -0.608\end{array}$
$\begin{array}{llll}H & -6.140 & 3.590 & -0.755\end{array}$
$\begin{array}{llll}\text { H } & -5.064 & 2.725 & -1.875\end{array}$
$\begin{array}{llll}C & -6.601 & 0.634 & -1.580\end{array}$
$\begin{array}{llll}\mathrm{H} & -7.209 & -0.267 & -1.693\end{array}$
H $-7.170 \quad 1.489-1.954$
H $-5.6990 .521 \quad-2.190$
$\begin{array}{lllll}\text { O } & -3.924 & 1.141 & -0.097\end{array}$ 


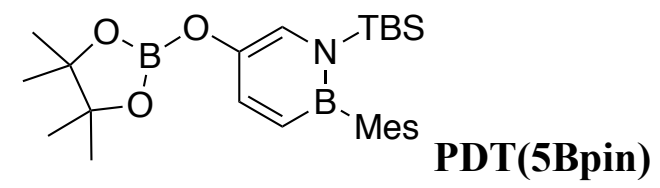

C $-2.091-0.926-1.403$

$\begin{array}{llll}\mathrm{H} & -3.121 & -0.797 & -1.731\end{array}$

$\begin{array}{llll}C & -1.673 & -2.239 & -1.054\end{array}$

$\begin{array}{llll}\text { O } & -2.543 & -3.309 & -1.172\end{array}$

$\begin{array}{llll}\text { B } & -3.718 & -3.322 & -0.480\end{array}$

$\begin{array}{llll}\text { O } & -4.588 & -4.375 & -0.580\end{array}$

$\begin{array}{llll}C & -5.809 & -3.948 & 0.071\end{array}$

$\begin{array}{llll}C & -6.462 & -5.150 & 0.731\end{array}$

$\begin{array}{llll}\mathrm{H} & -5.767 & -5.666 & 1.395\end{array}$

$\begin{array}{llll}H & -7.338 & -4.838 & 1.308\end{array}$

$\begin{array}{llll}\text { H } & -6.793 & -5.854 & -0.037\end{array}$

C $-5.290-2.856 \quad 1.065$

$\begin{array}{llll}C & -4.771 & -3.448 & 2.373\end{array}$

$\begin{array}{llll}\mathrm{H} & -4.082 & -4.276 & 2.181\end{array}$

$\begin{array}{llll}H & -5.592 & -3.813 & 2.996\end{array}$

$\begin{array}{llll}H & -4.235 & -2.671 & 2.922\end{array}$

$\begin{array}{llll}\text { C } & -6.269 & -1.728 & 1.340\end{array}$

$\begin{array}{llll}\mathrm{H} & -5.824 & -1.020 & 2.044\end{array}$

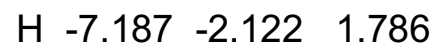

$\begin{array}{llll}H & -6.524 & -1.191 & 0.425\end{array}$

C $-6.715-3.370-1.013$

H $-6.874-4.129-1.783$

H $-7.686-3.079-0.603$

H $-6.256-2.494 \quad-1.481$

$\begin{array}{lllll}O & -4.143 & -2.322 & 0.358\end{array}$

$\begin{array}{llll}C & -0.397 & -2.490 & -0.653\end{array}$

$\begin{array}{llll}\mathrm{H} & -0.125 & -3.512 & -0.409\end{array}$

$\begin{array}{llll}\text { N } & 0.568 & -1.508 & -0.542\end{array}$

Si $\begin{array}{llll}2.222 & -2.162 & -0.155\end{array}$

C $1.989-3.423 \quad 1.223$

H $1.369-3.0022 .021$

H $2.965-3.667 \quad 1.654$

H $1.530 \quad-4.360 \quad 0.896$

$\begin{array}{llll}\text { C } & 3.435 & -0.871 & 0.456\end{array}$

H $3.092 \quad-0.395 \quad 1.377$

$\begin{array}{llll}\text { H } & 4.368 & -1.397 & 0.683\end{array}$

$\begin{array}{llll}\text { H } & 3.646 & -0.082 & -0.269\end{array}$

C $2.905-2.981-1.731$

C $4.277-3.591-1.402$

H 4.208 -4.344 -0.609

H $5.000-2.830-1.089$

H $4.687-4.086-2.292$

$\begin{array}{llll}\text { C } & 1.987 & -4.095 & -2.253\end{array}$

H $1.822 \quad-4.879-1.505$

$\begin{array}{llll}\text { H } & 1.012 & -3.708 & -2.564\end{array}$

H $2.448-4.571 \quad-3.129$

C $3.067-1.922-2.828$

$\begin{array}{llll}\text { H } & 3.727 & -1.105 & -2.514\end{array}$

H $2.099-1.489$-3.104

H $3.500-2.373-3.731$

$\begin{array}{llll}\text { B } & 0.215 & -0.131 & -0.826\end{array}$ 

C $1.208 \quad 1.073-0.557$
C $1.3551 .535 \quad 0.771$
$\begin{array}{llll}\text { C } & 0.589 & 0.873 & 1.894\end{array}$
$\begin{array}{llll}H & 0.738 & 1.400 & 2.839\end{array}$
$\begin{array}{llll}H & -0.484 & 0.849 & 1.678\end{array}$
H $0.902 \quad-0.169 \quad 2.037$
C $2.2042 .599 \quad 1.053$
H $2.3092 .942 \quad 2.081$
C $2.927 \quad 3.243 \quad 0.042$
$\begin{array}{lll}\text { C } 3.858 & 4.382 & 0.374\end{array}$
H $3.334 \quad 5.172 \quad 0.920$
H $4.6834 .043 \quad 1.009$
H $4.286 \quad 4.820-0.530$
$\begin{array}{llll}\text { C } & 2.747 & 2.810 & -1.267\end{array}$
H $3.276 \quad 3.317 \quad-2.073$
C $1.8881 .748-1.579$
$\begin{array}{llll}C & 1.721 & 1.374 & -3.034\end{array}$
$\begin{array}{llll}H & 0.926 & 0.637 & -3.176\end{array}$
$\begin{array}{llll}H & 1.471 & 2.257 & -3.631\end{array}$
$\begin{array}{llll}\text { H } & 2.644 & 0.956 & -3.447\end{array}$
$\begin{array}{llll}C & -1.213 & 0.115 & -1.306\end{array}$
H $-1.567 \quad 1.110-1.568$

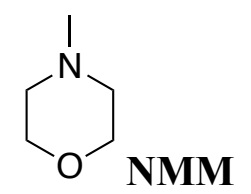

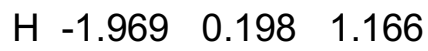

$\begin{array}{llll}H & 0.384 & 0.619 & 1.037\end{array}$

$\begin{array}{llll}H & -2.425 & 4.386 & 0.758\end{array}$

H $-0.681 \quad 4.698 \quad 0.660$

$\begin{array}{llll}\mathrm{N} & -1.239 & 2.696 & 0.419\end{array}$

$\begin{array}{lll}\text { C }-1.503 & 4.113 & 0.238\end{array}$

$\begin{array}{llll}\mathrm{H} & -3.260 & 2.155 & 0.416\end{array}$

$\begin{array}{llll}\text { C } & -2.033 & 0.415 & 0.089\end{array}$

$\begin{array}{lllll}C & 0.254 & 0.824 & -0.037\end{array}$

$\begin{array}{llll}\mathrm{H} & 0.831 & 2.887 & 0.188\end{array}$

C $-2.336 \quad 1.892 \quad-0.110$

$\begin{array}{llll}C & 0.004 & 2.310 & -0.240\end{array}$

$\begin{array}{llll}\text { H } & -2.819 & -0.203 & -0.351\end{array}$

$\begin{array}{lllll}\text { O } & -0.820 & 0.055 & -0.548\end{array}$

H $1.154 \quad 0.507 \quad-0.570$

$\begin{array}{llll}\mathrm{H} & -1.612 & 4.387 & -0.827\end{array}$

H $-2.4892 .087 \quad-1.189$

$\begin{array}{llll}H & -0.036 & 2.525 & -1.325\end{array}$ 


\subsection{Diethanolamine Transesterification}

All structures for the transesterification reaction were optimized at the M062X/6$31 \mathrm{G}(\mathrm{d}, \mathrm{p})$ level and computed using the polarized continuum model simulating THF. The coordinates are reproduced here.

Table S5. Gibbs free energies for diethanolamine transesterification reaction of 4Bpin and 5Bpin.

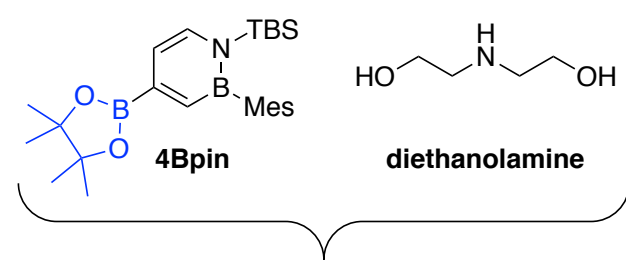

4 reactants

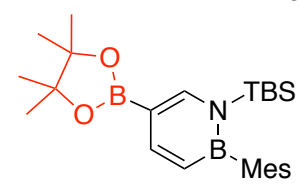

5Bpin

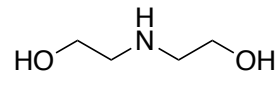

diethanolamine

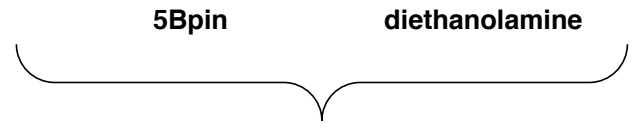

5 reactants
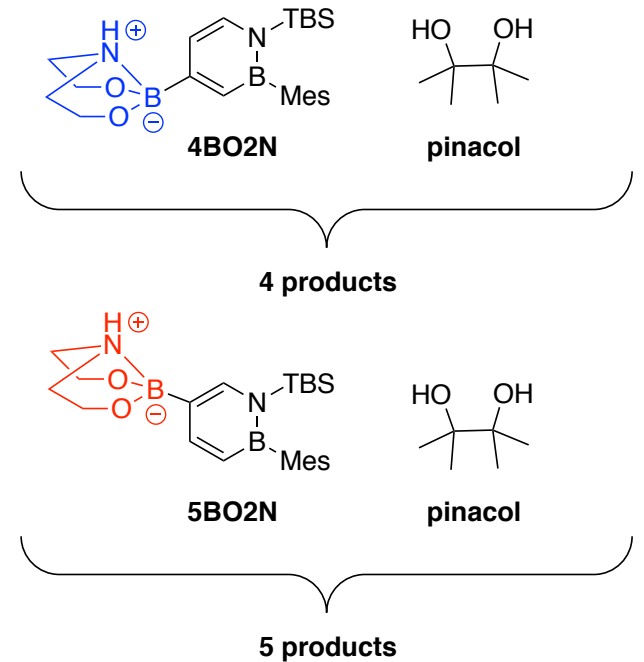

\begin{tabular}{ll} 
Structure & Gibbs Free Energy (Hartree) \\
\hline 4Bpin & -1520.9036 \\
diethanolamine & -363.937278 \\
4 reactants & -1884.840878 \\
4BO2N & -1497.654746 \\
pinacol & -387.195773 \\
4 products & -1884.850519 \\
5Bpin & -1520.908741 \\
5 reactants & -1884.846019 \\
5BO2N & -1497.658469 \\
5 products & -1884.854242
\end{tabular}

4 reactants - products $=-6.05 \mathrm{kcal} \mathrm{mol}^{-1}$ 5 reactants - products $=-5.16 \mathrm{kcal} \mathrm{mol}^{-1}$ 4 products favored by $0.89 \mathrm{kcal} \mathrm{mol}^{-1}$ 
Figure S13. Reaction coordinates for optimized geometries for diethanolamine transesterification.<smiles></smiles>
B $-3.462 \quad-0.298 \quad 0.013$
$\begin{array}{llll}\text { O } & -4.420 & -1.267 & 0.166\end{array}$
C $-5.690-0.659-0.173$
C $-6.778-1.263 \quad 0.698$
H $-6.526-1.191 \quad 1.758$
H $-7.730-0.752 \quad 0.526$
$\begin{array}{llll}\mathrm{H} & -6.906 & -2.319 & 0.445\end{array}$
$\begin{array}{llll}C & -5.408 & 0.857 & 0.088\end{array}$
C $-\begin{array}{llll}-5.613 & 1.253 & 1.548\end{array}$
$\begin{array}{llll}\mathrm{H} & -5.091 & 0.563 & 2.217\end{array}$
H $-6.674 \quad 1.262 \quad 1.811$
H $\quad-5.206 \quad 2.255 \quad 1.701$
$\begin{array}{llll}C & -6.150 & 1.810 & -0.833\end{array}$
$\begin{array}{llll}\mathrm{H} & -5.891 & 2.841 & -0.578\end{array}$
$\begin{array}{llll}\mathrm{H} & -7.231 & 1.689 & -0.712\end{array}$
H $-5.891 \quad 1.638-1.878$
C $-5.949-0.969-1.645$
H $-5.929-2.053-1.785$
$\begin{array}{llll}\mathrm{H} & -6.924 & -0.594 & -1.965\end{array}$
H $-5.176-0.525 \quad-2.279$
$\begin{array}{llll}C & -1.928 & -0.601 & 0.049\end{array}$
$\begin{array}{llll}C & -1.483 & -1.950 & 0.215\end{array}$
$\begin{array}{llll}\text { H } & -2.206 & -2.751 & 0.328\end{array}$
$\begin{array}{llll}C & -0.157 & -2.256 & 0.231\end{array}$
H $0.151 \quad-3.291 \quad 0.356$
$\begin{array}{llll}\text { N } & 0.852 & -1.321 & 0.100\end{array}$
$\begin{array}{llll}\text { B } & 0.500 & 0.081 & -0.020\end{array}$
C $1.583 \quad 1.236 \quad 0.013$
C $2.1181 .617 \quad 1.265$
C $1.6800 .905 \quad 2.525$
H $2.109 \quad 1.374 \quad 3.413$
$\begin{array}{llll}H & 0.590 & 0.914 & 2.625\end{array}$
H $1.986-0.148 \quad 2.519$
C $3.0512 .644 \quad 1.346$
H $3.456 \quad 2.924 \quad 2.317$
C $3.482 \quad 3.330 \quad 0.204$
C $4.494 \quad 4.442 \quad 0.317$
H $4.075 \quad 5.3010 .852$
$\begin{array}{llll}H & 5.378 & 4.115 & 0.873\end{array}$
H $4.818 \quad 4.785 \quad-0.668$
C $2.9272 .975-1.019$
H $3.230 \quad 3.513 \quad-1.917$
C $1.976 \quad 1.951-1.127$
C $1.4001 .655-2.493$
$\begin{array}{llll}H & 0.541 & 0.981 & -2.434\end{array}$
H $1.071 \quad 2.577 \quad-2.981$ 

H $2.146 \quad 1.192 \quad-3.147$
$\begin{array}{llll}\text { Si } & 2.510 & -2.056 & -0.030\end{array}$
$\begin{array}{llll}\text { C } & 2.631 & -3.368 & 1.314\end{array}$
$\begin{array}{llll}\text { H } & 2.252 & -2.978 & 2.264\end{array}$
H $3.681-3.637 \quad 1.461$
H $2.084-4.287 \quad 1.085$
$\begin{array}{llll}\text { C } & 3.913 & -0.840 & 0.229\end{array}$
H $3.879-0.381 \quad 1.219$
$\begin{array}{llll}\text { H } & 4.845 & -1.412 & 0.161\end{array}$
H $3.942-0.038-0.512$
C $2.662-2.837-1.761$
$\begin{array}{llll}C & 4.027 & -3.537 & -1.853\end{array}$
H $4.130-4.329-1.103$
$\begin{array}{llll}\text { H } & 4.858 & -2.835 & -1.724\end{array}$
H $4.142 \quad-4.001-2.841$
C $1.561-3.871-2.035$
H $1.559-4.678$-1.293
H $0.566-3.415-2.046$
$\begin{array}{llll}\text { H } & 1.725 & -4.330 & -3.018\end{array}$
$\begin{array}{llll}\text { C } 2.577 & -1.736 & -2.826\end{array}$
H $3.360-0.980-2.697$
$\begin{array}{llll}\text { H } & 1.607 & -1.228 & -2.792\end{array}$
H $2.693-2.168$-3.828
$\begin{array}{llll}C & -0.993 & 0.399 & -0.066\end{array}$
H $-1.354 \quad 1.421 \quad-0.179$
$\begin{array}{lllll}\text { O } & -3.987 & 0.953 & -0.178\end{array}$<smiles></smiles>

$\begin{array}{llll}\text { B } & -3.461 & -0.300 & 0.010\end{array}$

$\begin{array}{llll}\text { O } & -4.421 & -1.266 & 0.163\end{array}$

$\begin{array}{llll}C & -5.690 & -0.655 & -0.174\end{array}$

$\begin{array}{llll}C & -6.778 & -1.253 & 0.702\end{array}$

$\begin{array}{llll}\mathrm{H} & -6.516 & -1.188 & 1.759\end{array}$

$\begin{array}{llll}\mathrm{H} & -7.726 & -0.731 & 0.541\end{array}$

$\begin{array}{llll}\text { H } & -6.920 & -2.306 & 0.445\end{array}$

$\begin{array}{llll}C & -5.403 & 0.859 & 0.086\end{array}$

$\begin{array}{llll}C & -5.605 & 1.254 & 1.547\end{array}$

$\begin{array}{llll}\mathrm{H} & -5.088 & 0.560 & 2.215\end{array}$

H $-6.666 \quad 1.267 \quad 1.809$

H $\quad-5.193 \quad 2.254 \quad 1.705$

$\begin{array}{llll}\text { C } & -6.146 & 1.815 & -0.832\end{array}$

$\begin{array}{llll}\mathrm{H} & -5.887 & 2.844 & -0.573\end{array}$

$\begin{array}{llll}\mathrm{H} & -7.227 & 1.694 & -0.713\end{array}$

H $-5.887 \quad 1.646-1.879$

$\begin{array}{llll}C & -5.957 & -0.966 & -1.644\end{array}$

$\begin{array}{llll}\mathrm{H} & -5.932 & -2.050 & -1.785\end{array}$

$\begin{array}{llll}H & -6.936 & -0.597 & -1.958\end{array}$

$\begin{array}{llll}H & -5.191 & -0.517 & -2.283\end{array}$

$\begin{array}{llll}C & -1.927 & -0.604 & 0.042\end{array}$ 

C $-1.483 \quad-1.953 \quad 0.210$
$\begin{array}{llll}\mathrm{H} & -2.206 & -2.754 & 0.322\end{array}$
$\begin{array}{llll}C & -0.155 & -2.259 & 0.231\end{array}$
$\begin{array}{llll}\mathrm{H} & 0.152 & -3.293 & 0.358\end{array}$
$\begin{array}{llll}\text { N } & 0.854 & -1.324 & 0.098\end{array}$
$\begin{array}{llll}\text { B } & 0.500 & 0.078 & -0.026\end{array}$
C $1.582 \quad 1.234 \quad 0.007$
C $2.119 \quad 1.609 \quad 1.260$
$\begin{array}{llll}\text { C } & 1.687 & 0.890 & 2.517\end{array}$
H $2.109 \quad 1.361 \quad 3.407$
$\begin{array}{llll}H & 0.596 & 0.880 & 2.618\end{array}$
H $2.007 \quad-0.159 \quad 2.510$
C $3.048 \quad 2.639 \quad 1.342$
H $3.450 \quad 2.921 \quad 2.313$
C $3.468 \quad 3.335 \quad 0.203$
C $4.475 \quad 4.4520 .316$
$\begin{array}{llll}\mathrm{H} & 4.060 & 5.303 & 0.866\end{array}$
$\begin{array}{llll}H & 5.368 & 4.123 & 0.857\end{array}$
H $4.786 \quad 4.806 \quad-0.669$
C $2.914 \quad 2.981 \quad-1.021$
H $3.213 \quad 3.525 \quad-1.917$
C $1.971 \quad 1.953-1.132$
$\begin{array}{llll}C & 1.395 & 1.658 & -2.499\end{array}$
$\begin{array}{llll}H & 0.525 & 1.000 & -2.442\end{array}$
H $1.0852 .586-2.992$
H $2.136 \quad 1.181 \quad-3.150$
Si $2.513-2.059-0.026$
$\begin{array}{llll}\text { C } & 2.627 & -3.372 & 1.317\end{array}$
H $2.255 \quad-2.982 \quad 2.270$
H $3.677 \quad-3.650 \quad 1.460$
H $2.074 \quad-4.287 \quad 1.087$
$\begin{array}{llll}\text { C } & 3.918 & -0.846 & 0.235\end{array}$
H $3.883-0.3851 .223$
$\begin{array}{llll}\text { H } & 4.848 & -1.421 & 0.171\end{array}$
H $3.952-0.045 \quad-0.506$
$\begin{array}{llll}\text { C } & 2.667 & -2.837 & -1.756\end{array}$
$\begin{array}{llll}\text { C } & 4.033 & -3.537 & -1.847\end{array}$
H $4.133-4.329-1.096$
H $4.864-2.835-1.715$
H $4.150-4.003 \quad-2.834$
$\begin{array}{llll}\text { C } & 1.568 & -3.872 & -2.033\end{array}$
$\begin{array}{llll}\text { H } & 1.565 & -4.680 & -1.293\end{array}$
H $0.572 \quad-3.420 \quad-2.049$
$\begin{array}{llll}\text { H } & 1.735 & -4.332 & -3.016\end{array}$
$\begin{array}{llll}\text { C } & 2.583 & -1.735 & -2.819\end{array}$
$\begin{array}{llll}\text { H } & 3.361 & -0.975 & -2.685\end{array}$
H $1.610-1.232 \quad-2.789$
H $2.706-2.164 \quad-3.822$
$\begin{array}{llll}\text { C } & -0.991 & 0.396 & -0.071\end{array}$
$\begin{array}{llll}\mathrm{H} & -1.353 & 1.419 & -0.179\end{array}$
$\begin{array}{lllll}\text { O } & -3.983 & 0.952 & -0.182\end{array}$ 

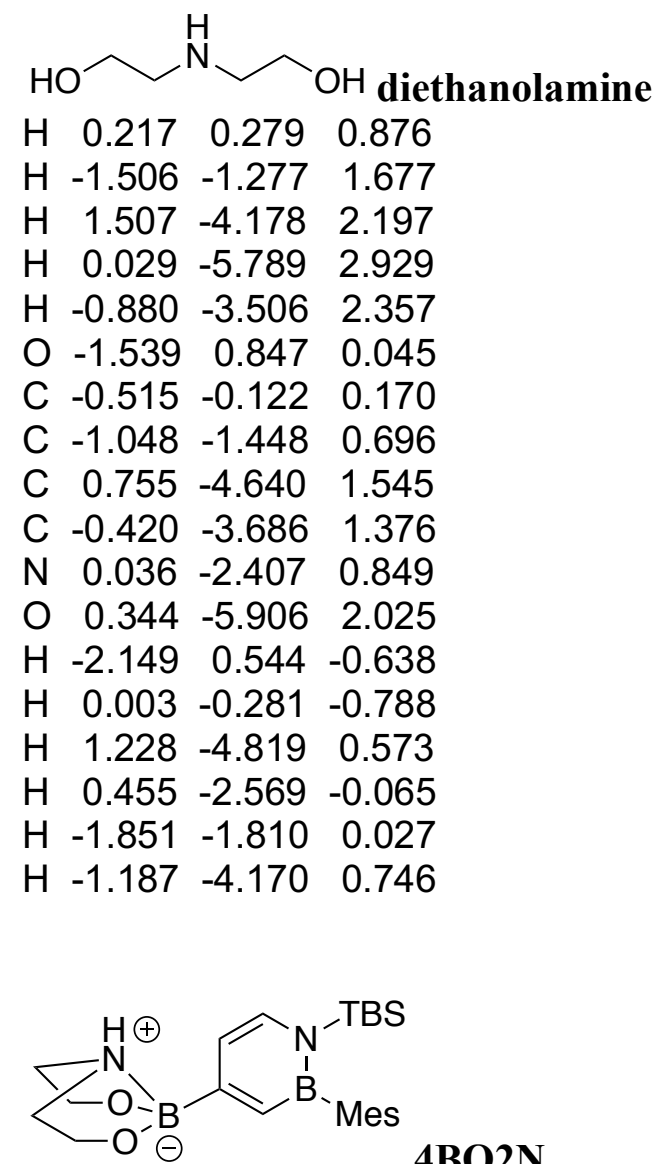
H $1.4351 .442 \quad 3.373$
$\begin{array}{llll}H & 0.010 & 0.997 & 2.414\end{array}$
$\begin{array}{llll}\text { H } & 1.692 & -2.957 & 2.326\end{array}$
$\begin{array}{llll}\mathrm{H} & -3.650 & 1.875 & 1.377\end{array}$
C $1.104 \quad 0.963 \quad 2.448$
H $-3.953 \quad-0.340 \quad 1.673$
H $-5.731-1.597 \quad 1.416$
H $\quad \begin{array}{llll}\text { H } & -381 & -0.097 & 2.499\end{array}$
C $2.118-3.371 \quad 1.406$
$\begin{array}{llll}\mathrm{N} & -4.517 & 0.041 & 0.917\end{array}$
H $1.541 \quad-4.2601 .137$
$\begin{array}{llll}C & -4.620 & 1.512 & 1.029\end{array}$
$\begin{array}{llll}C & -5.802 & -0.704 & 0.795\end{array}$
H $3.139-3.696 \quad 1.631$
$\begin{array}{llll}H & -2.564 & -2.665 & -0.166\end{array}$
H $2.936 \quad 2.941 \quad 2.418$
$\begin{array}{llll}C & -1.818 & -1.876 & -0.191\end{array}$
$\begin{array}{llll}\mathrm{H} & -0.245 & -3.247 & 0.187\end{array}$
$\begin{array}{llll}\text { H } & -6.476 & -2.009 & -0.824\end{array}$
$\begin{array}{llll}\text { C } & -0.513 & -2.208 & 0.007\end{array}$
H $-4.576 \quad 3.002 \quad-0.554$
C $\quad 1.704 \quad 1.6391 .237$
H $\quad-5.400 \quad 1.816 \quad 1.730$
$\begin{array}{llll}\text { C } & -4.857 & 1.953 & -0.414\end{array}$
H $-6.639-0.094 \quad 1.144$
$\begin{array}{llll}\text { C } & -5.895 & -1.092 & -0.693\end{array}$
H $3.407-0.411 \quad 1.478$
$\begin{array}{llll}\text { C } & -2.223 & -0.526 & -0.447\end{array}$ 

C $2.6412 .652 \quad 1.410$
$\begin{array}{llll}\text { B } & -3.781 & -0.186 & -0.617\end{array}$
$\begin{array}{llll}\text { N } & 0.526 & -1.296 & -0.006\end{array}$
$\begin{array}{llll}\text { Si } & 2.170 & -2.055 & 0.061\end{array}$
$\begin{array}{llll}B & 0.211 & 0.109 & -0.207\end{array}$
$\begin{array}{llll}\text { C } & -1.252 & 0.447 & -0.459\end{array}$
$\begin{array}{llll}\mathrm{H} & -5.924 & 1.857 & -0.671\end{array}$
$\begin{array}{llll}C & 3.555 & -0.868 & 0.497\end{array}$
H $-6.379-0.292-1.270$
C $1.308 \quad 1.246-0.063$
$\begin{array}{lllll}O & -4.056 & 1.117 & -1.206\end{array}$
$\begin{array}{lllll}O & -4.568 & -1.291 & -1.105\end{array}$
$\mathrm{H}-1.576 \quad 1.470-0.654$
H $4.476-1.4590 .542$
H 1.403 -4.691 -1.278
$\begin{array}{llll}\text { C } & 3.212 & 3.313 & 0.317\end{array}$
$\begin{array}{llll}\text { H } & 3.950 & -4.287 & -0.833\end{array}$
H $3.807 \quad 5.237 \quad 1.093$
H $5.091 \quad 4.031 \quad 1.099$
C $4.233 \quad 4.401 \quad 0.529$
$\begin{array}{llll}C & 1.468 & -3.887 & -2.020\end{array}$
$\begin{array}{llll}\text { H } & 3.691 & -0.064 & -0.229\end{array}$
$\begin{array}{llll}\text { C } & 2.518 & -2.833 & -1.642\end{array}$
$\begin{array}{llll}\text { H } & 0.472 & -3.447 & -2.135\end{array}$
C $3.899-3.506-1.600$
C $1.8381 .941-1.159$
$\begin{array}{llll}C & 2.790 & 2.950 & -0.957\end{array}$
H $4.700-2.785$-1.407
H 1.738 -4.348 $\quad-2.979$
$\begin{array}{llll}H & 4.601 & 4.790 & -0.423\end{array}$
$\begin{array}{llll}\text { C } & 2.517 & -1.733 & -2.709\end{array}$
$\begin{array}{llll}\text { H } & 4.111 & -3.980 & -2.567\end{array}$
H $0.5390 .982 \quad-2.606$
$\begin{array}{llll}\text { C } & 1.407 & 1.646 & -2.577\end{array}$
$\begin{array}{llll}\text { H } & 1.538 & -1.245 & -2.774\end{array}$
$\begin{array}{llll}\text { H } & 3.266 & -0.960 & -2.499\end{array}$
$\begin{array}{llll}\text { H } & 3.201 & 3.470 & -1.821\end{array}$
H $1.142 \quad 2.571 \quad-3.099$
H $2.212 \quad 1.173 \quad-3.149$
$\begin{array}{llll}\text { H } & 2.745 & -2.158 & -3.696\end{array}$

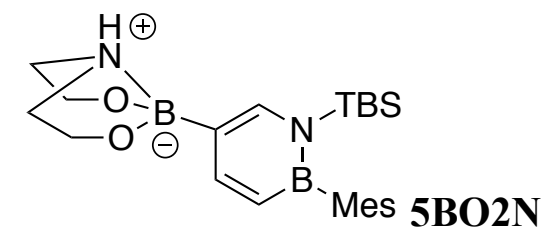

B -3.205 $-3.033-1.578$

$\begin{array}{llll}\mathrm{N} & -4.105 & -3.431 & -0.157\end{array}$

$\begin{array}{llll}\mathrm{H} & -3.648 & -3.089 & 0.683\end{array}$

$\begin{array}{llll}C & -4.136 & -4.919 & -0.165\end{array}$

$\begin{array}{llll}\mathrm{H} & -3.433 & -5.270 & 0.592\end{array}$

$\begin{array}{llll}\text { H } & -5.134 & -5.293 & 0.073\end{array}$

$\begin{array}{llll}\text { C } & -3.639 & -5.313 & -1.566\end{array}$ 

H -3.182 $-6.307-1.555$
H $-4.472-5.330-2.283$
C $-5.402-2.749-0.359$
$\begin{array}{llll}\text { H } & -6.211 & -3.240 & 0.187\end{array}$
H -5.288 -1.724 0.001
$\begin{array}{llll}\text { C }-5.571 & -2.752 & -1.878\end{array}$
H -6.262 $-1.964-2.195$
H $-5.988-3.714-2.217$
C $-2.056-1.965-1.293$
C $-0.800-2.392-0.957$
N 0.277 -1.574 -0.676
Si $1.844-2.477-0.606$
$\begin{array}{llll}\text { C } & 1.636 & -3.890 & 0.619\end{array}$
H $1.469-3.4911 .624$
$\begin{array}{llll}\text { H } & 2.554 & -4.486 & 0.649\end{array}$
H $\quad 0.811 \quad-4.5640 .380$
$\begin{array}{llll}\text { C } & 3.295 & -1.440 & -0.030\end{array}$
H $3.126-1.0020 .956$
$\begin{array}{llll}\text { H } & 4.159 & -2.110 & 0.041\end{array}$
H $3.542-0.626-0.717$
C $2.195-3.121-2.368$
C $3.665-3.566-2.438$
H $3.895-4.345-1.701$
H $4.353-2.731-2.274$
H $3.880-3.982-3.431$
C $1.310-4.316-2.754$
H $1.420-5.149-2.050$
H $\quad 0.249-4.052-2.805$
H $1.603-4.684-3.747$
C $1.969-1.984-3.374$
H $2.585-1.108-3.141$
$\begin{array}{llll}H & 0.920 & -1.665 & -3.387\end{array}$
$\begin{array}{llll}\text { H } & 2.234 & -2.316 & -4.387\end{array}$
B $0.083-0.134-0.675$
$\begin{array}{llll}\text { C } & 1.225 & 0.876 & -0.237\end{array}$
C $1.509 \quad 1.0351 .139$
$\begin{array}{llll}\text { C } & 0.772 & 0.206 & 2.165\end{array}$
$\begin{array}{llll}\text { H } & 1.011 & 0.528 & 3.181\end{array}$
$\begin{array}{llll}H & 1.031 & -0.857 & 2.080\end{array}$
$\begin{array}{llll}\text { H } & -0.312 & 0.276 & 2.025\end{array}$
$\begin{array}{llll}C & 2.478 & 1.942 & 1.556\end{array}$
H 2.6862 .0502 .620
C $3.193 \quad 2.7190 .638$
C 4.2503 .6831 .114
H $3.828 \quad 4.422 \quad 1.802$
H $5.047 \quad 3.1601 .651$
$\begin{array}{llll}\text { H } & 4.702 & 4.220 & 0.277\end{array}$
$\begin{array}{llll}C & 2.892 & 2.578 & -0.711\end{array}$
H $3.427 \quad 3.183-1.442$
C $1.9121 .680-1.156$
$\begin{array}{llll}C & 1.629 & 1.617 & -2.641\end{array}$
$\begin{array}{llll}\text { H } & 0.890 & 0.850 & -2.883\end{array}$
$\begin{array}{llll}H & 1.246 & 2.577 & -3.002\end{array}$
$\begin{array}{llll}\text { H } & 2.541 & 1.401 & -3.207\end{array}$
$\begin{array}{llll}\text { C } & -1.307 & 0.355 & -1.053\end{array}$ 
$\begin{array}{llll}H & -1.547 & 1.416 & -1.107\end{array}$

$\begin{array}{llll}\text { H } & -0.620 & -3.463 & -0.913\end{array}$

C $-2.285-0.554-1.352$

H -3.284 $-0.231-1.651$

$\begin{array}{lllll}\text { O } & -2.686 & -4.341 & -1.907\end{array}$

$\begin{array}{llll}\text { O } & -4.291 & -2.521 & -2.401\end{array}$

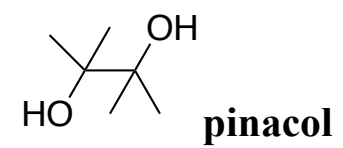

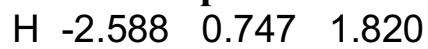

$\begin{array}{llll}\mathrm{O} & -3.498 & 0.604 & 1.519\end{array}$

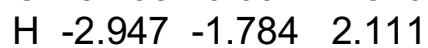

$\begin{array}{llll}0 & -1.091 & -0.346 & 0.869\end{array}$

$\begin{array}{llll}C & -2.566 & -2.152 & 1.155\end{array}$

$\begin{array}{llll}\mathrm{H} & -0.643 & 0.200 & 0.211\end{array}$

$\begin{array}{llll}\text { C } & -3.371 & 0.070 & 0.205\end{array}$

$\begin{array}{llll}\mathrm{H} & -2.082 & 1.664 & -0.552\end{array}$

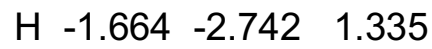

$\begin{array}{llll}C & -2.217 & -0.982 & 0.241\end{array}$

$\begin{array}{llll}C & -3.059 & 1.216 & -0.764\end{array}$

$\begin{array}{llll}\mathrm{H} & -3.813 & 1.995 & -0.634\end{array}$

$\begin{array}{llll}\text { H } & -3.319 & -2.797 & 0.698\end{array}$

$\begin{array}{llll}\text { C } & -4.724 & -0.542 & -0.138\end{array}$

$\begin{array}{llll}\text { H } & -5.021 & -1.275 & 0.615\end{array}$

$\begin{array}{llll}\mathrm{H} & -5.480 & 0.248 & -0.158\end{array}$

C $-1.813-1.486-1.141$

$\begin{array}{llll}\mathrm{H} & -3.075 & 0.890 & -1.807\end{array}$

$\begin{array}{llll}\mathrm{H} & -1.030 & -2.242 & -1.037\end{array}$

H $-1.425-0.675-1.766$

H $-4.708-1.024-1.119$

H $-2.660-1.939-1.663$ 


\section{NMR Spectra}

\begin{tabular}{|c|c|}
\hline Parameter & Value \\
\hline 1 Title & CRM-3-231-1H-3 \\
\hline 2 Comment & C4/ C5Bpin ratio determination \\
\hline 3 Solvent & c6d6 \\
\hline 4 Temperature & 25.0 \\
\hline 5 Relaxation/Delay & 15.0000 \\
\hline 6 Acquisition Date & 2018-10-30T12:31:34 \\
\hline 7 Spectrometer Freq & 499.88 \\
\hline 8 Nucleus & $1 H$ \\
\hline
\end{tabular}

S.S.
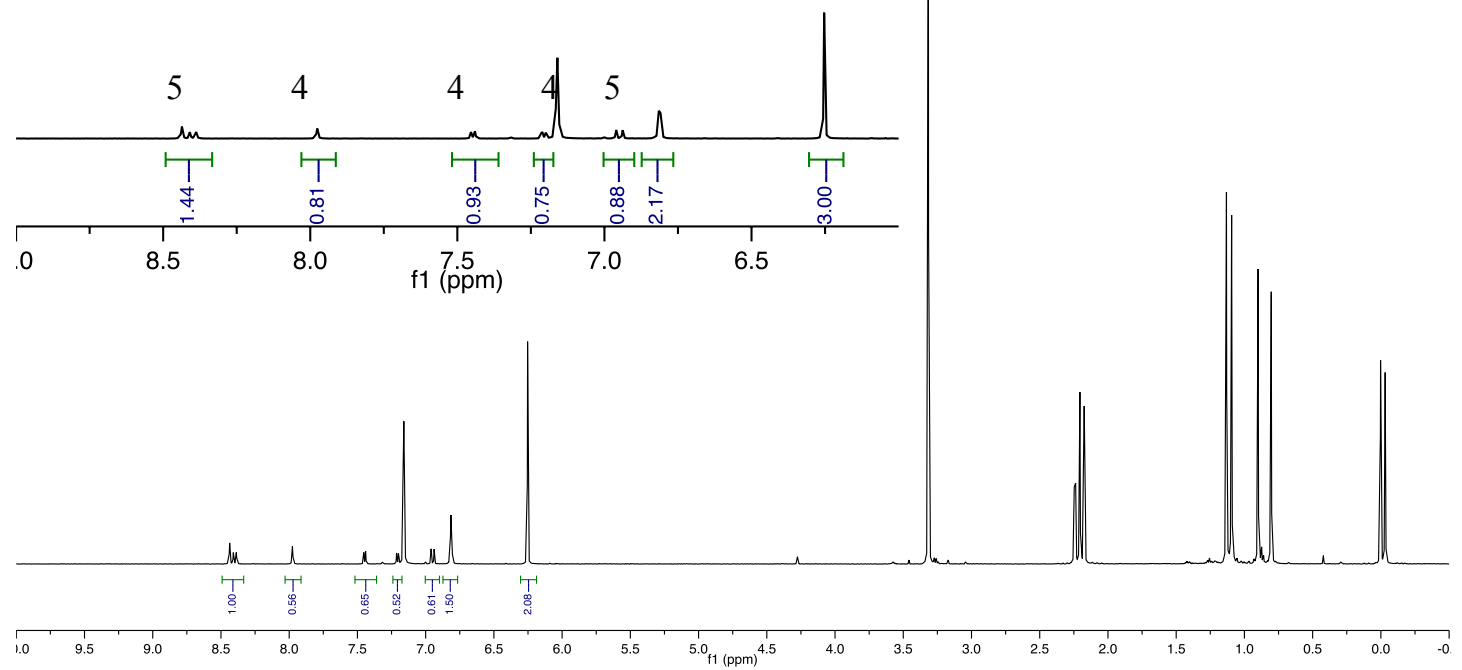

Figure S14. ${ }^{1} \mathrm{H}$ NMR of mixture of 5 pin and 4Bpin in $\mathrm{C}_{6} \mathrm{D}_{6}$ with 1,3,5trimethoxybenzene as internal standard. 


\begin{tabular}{|ll|}
1 Title & \multicolumn{1}{c|}{ Value } \\
2 Comment & CRM-3-177-1H-3 \\
3 Solvent & C5Bpin pure \\
4 Temperature & C6d6 \\
5 Relaxation Delay & 25.0 \\
6 Acquisition Date & 1.0000 \\
7 Spectrometer Frequency & $2018-07-05 T 10: 05: 26$ \\
8 Nucleus & $1 \mathrm{H}$ \\
\hline
\end{tabular}

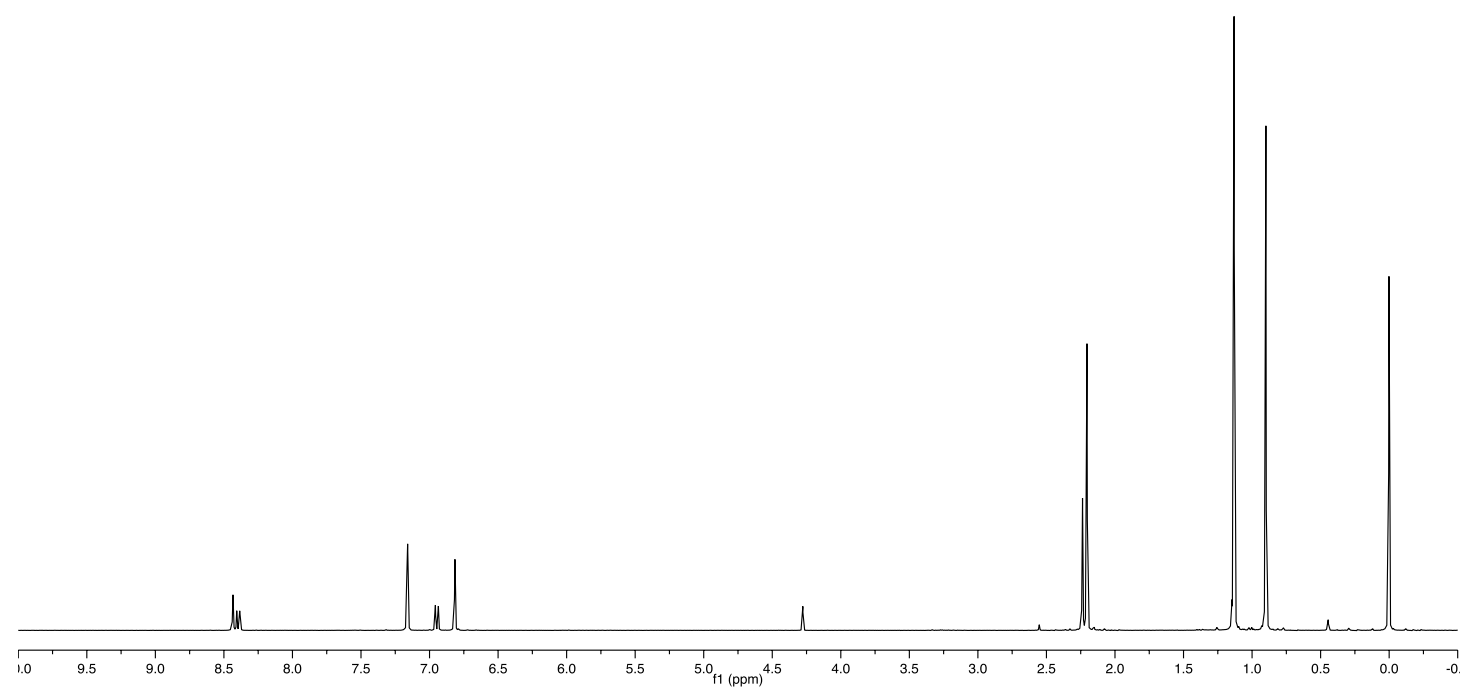

Figure S15. ${ }^{1} \mathrm{H}$ NMR of 5pin in $\mathrm{C}_{6} \mathrm{D}_{6}$.

\begin{tabular}{|ll|}
\multicolumn{1}{c|}{ Parameter } & \multicolumn{1}{c|}{ Value } \\
1 Title & CRM-3-067-1H-5 \\
2 Comment & C4Bpin column \\
3 Solvent & C6d6 \\
4 Temperature & 25.0 \\
5 Relaxation Delay & 1.0000 \\
6 Acquisition Date & $2018-03-08 \mathrm{~T} 16: 31: 07$ \\
7 Spectrometer Frequency & 399.77 \\
8 Nucleus & $1 \mathrm{H}$ \\
\hline
\end{tabular}

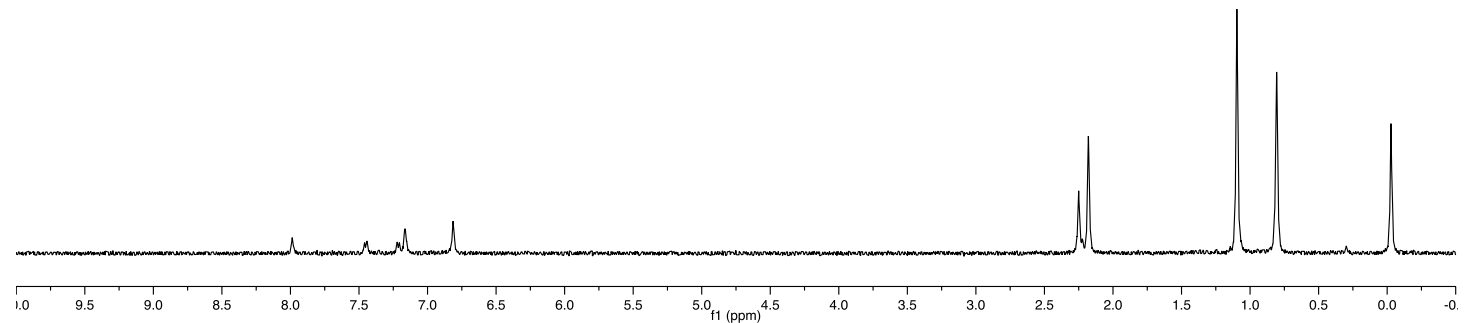

Figure S16. ${ }^{1} \mathrm{H}$ NMR of 4 pin in $\mathrm{C}_{6} \mathrm{D}_{6}$. 


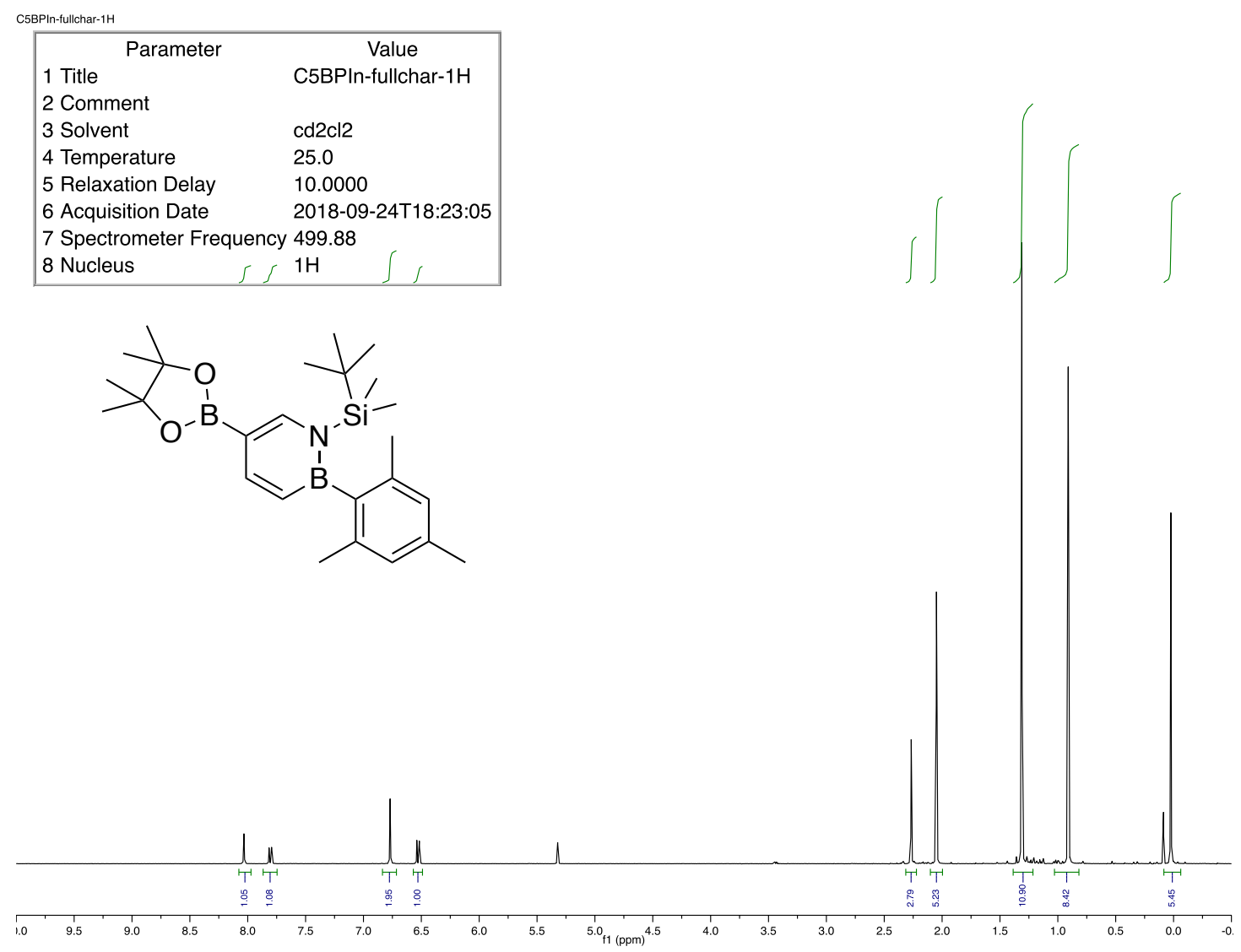

\begin{tabular}{|ll|}
\hline \multicolumn{1}{|c|}{ Parameter } & \multicolumn{1}{c|}{ Value } \\
1 Title & C5BPIn-fullchar-13C \\
2 Comment & cd2cl2 \\
3 Solvent & 25.0 \\
4 Temperature & 1.0000 \\
5 Relaxation Delay & $2018-09-24 T 18: 24: 53$ \\
6 Acquisition Date & 125.71 \\
7 Spectrometer Frequency & $13 \mathrm{C}$ \\
8 Nucleus & $13 \mathrm{C}$ \\
\hline
\end{tabular}<smiles>Cc1cc(C)c(B2C=CC(B3OC(C)(C)C(C)(C)O3)=CN2[Si](C)(C)C(C)(C)C)c(C)c1</smiles>

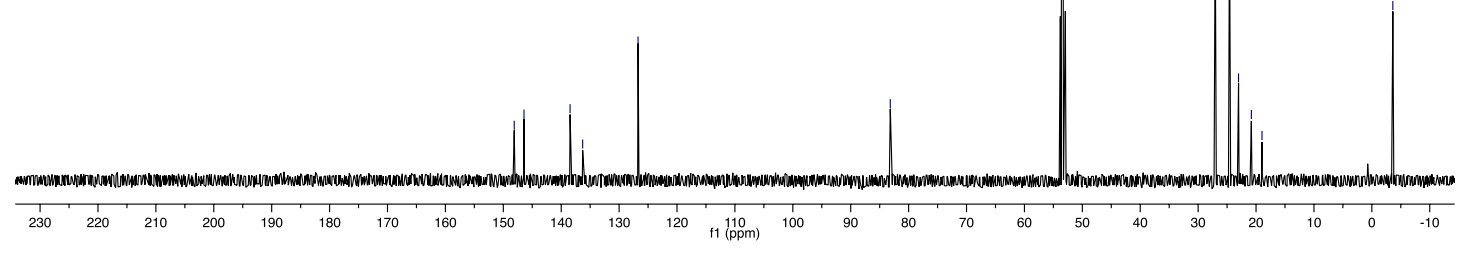




\begin{tabular}{|c|c|}
\hline \multicolumn{2}{|l|}{ C5Bpin-fullchar-11B } \\
\hline Parameter & Value $\stackrel{0}{~ \mid ~}$ \\
\hline 1 Title & C5Bpin-fullchar-11B \\
\hline \multicolumn{2}{|l|}{2 Comment } \\
\hline 3 Solvent & $\mathrm{cd} 2 \mathrm{cl} 2$ \\
\hline 4 Temperature & 25.0 \\
\hline 5 Relaxation Delay & 0.0100 \\
\hline 6 Acquisition Date & 2018-09-24T18:43:28 \\
\hline \multicolumn{2}{|c|}{7 Spectrometer Frequency 160.35} \\
\hline 8 Nucleus & 11B \\
\hline
\end{tabular}<smiles>Cc1cc(C)c(B2C=CC(B3OC(C)(C)C(C)(C)O3)=CN2[Si](C)(C)C(C)(C)C)c(C)c1</smiles>

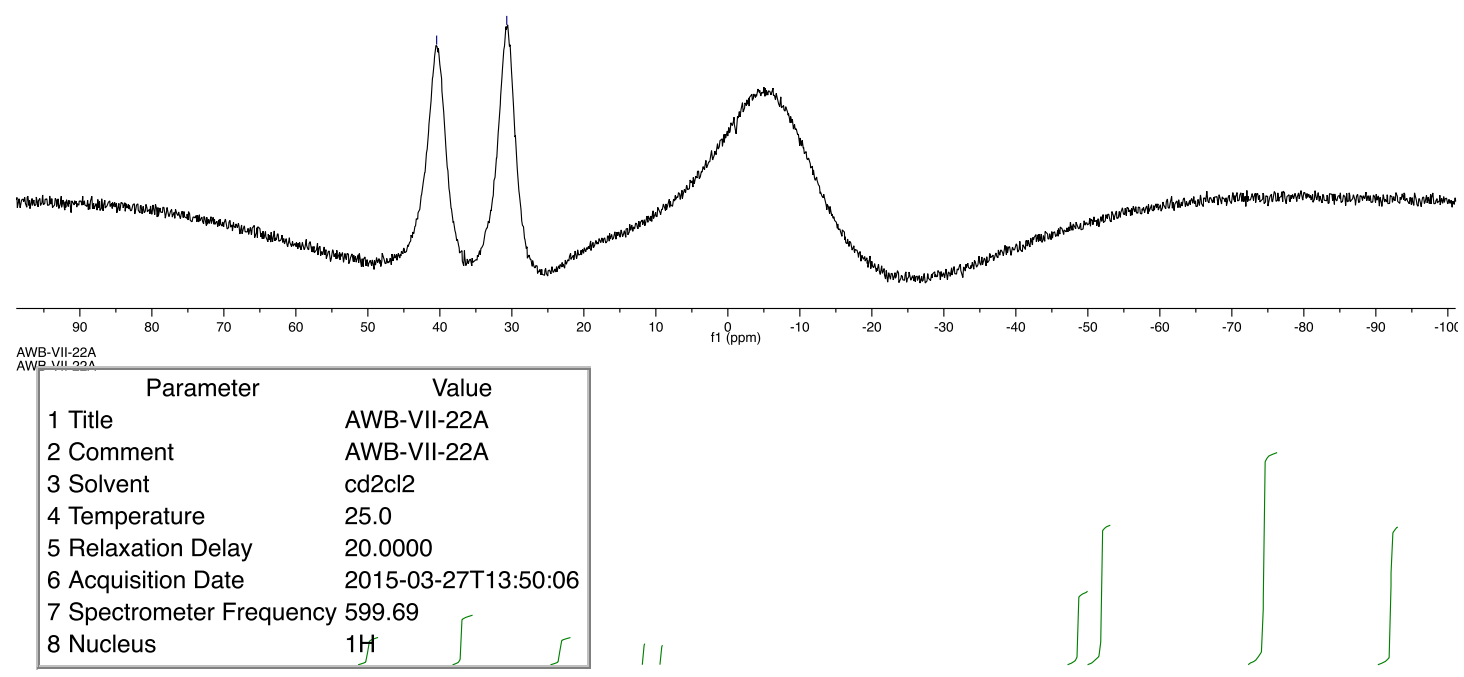<smiles>Cc1cc(C)c(B2C=C(O)C=CN2[Si](C)(C)C(C)(C)C)c(C)c1</smiles>

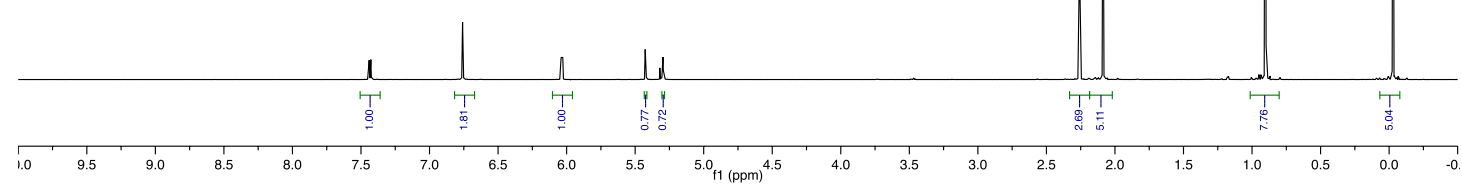




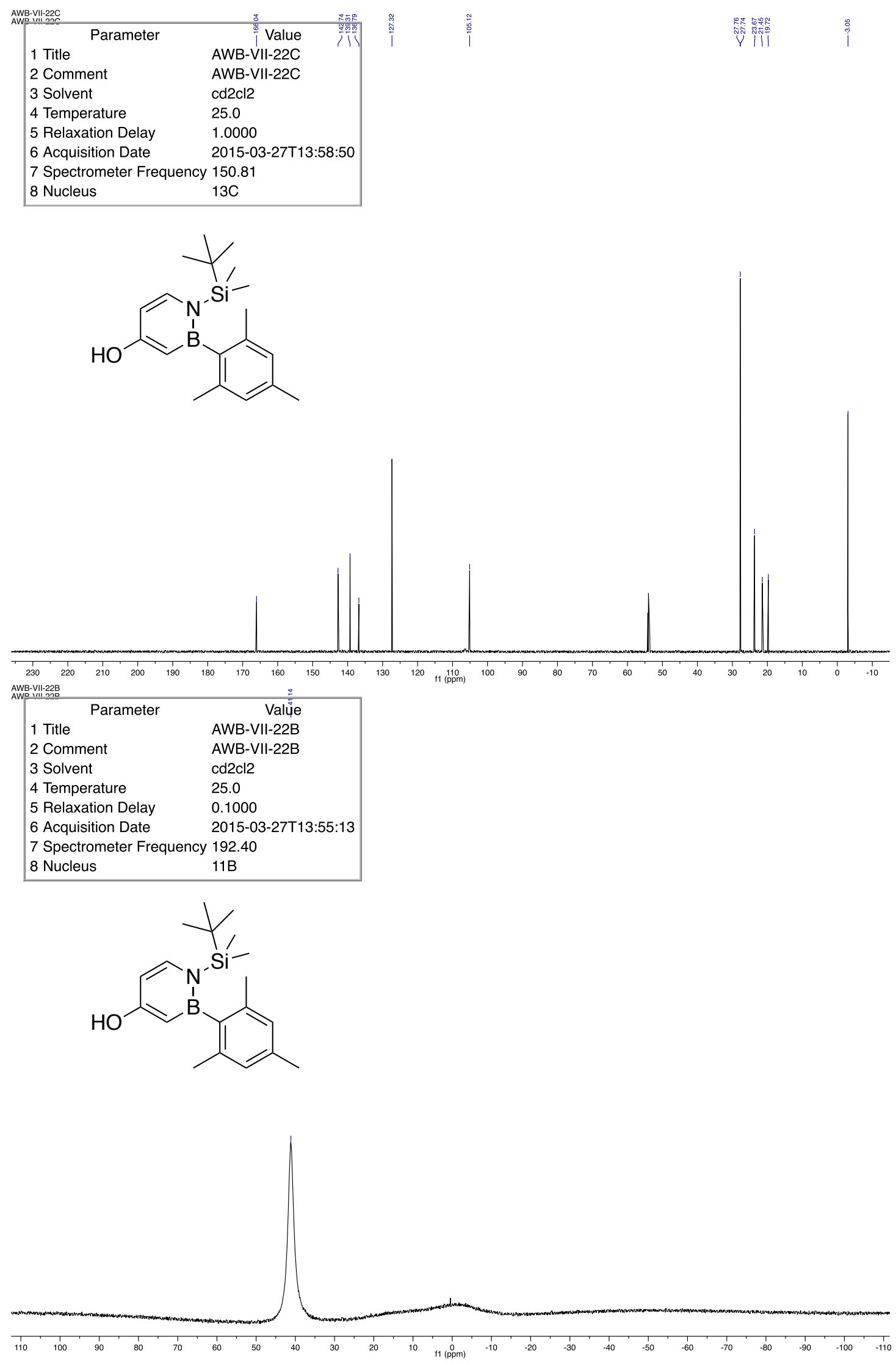




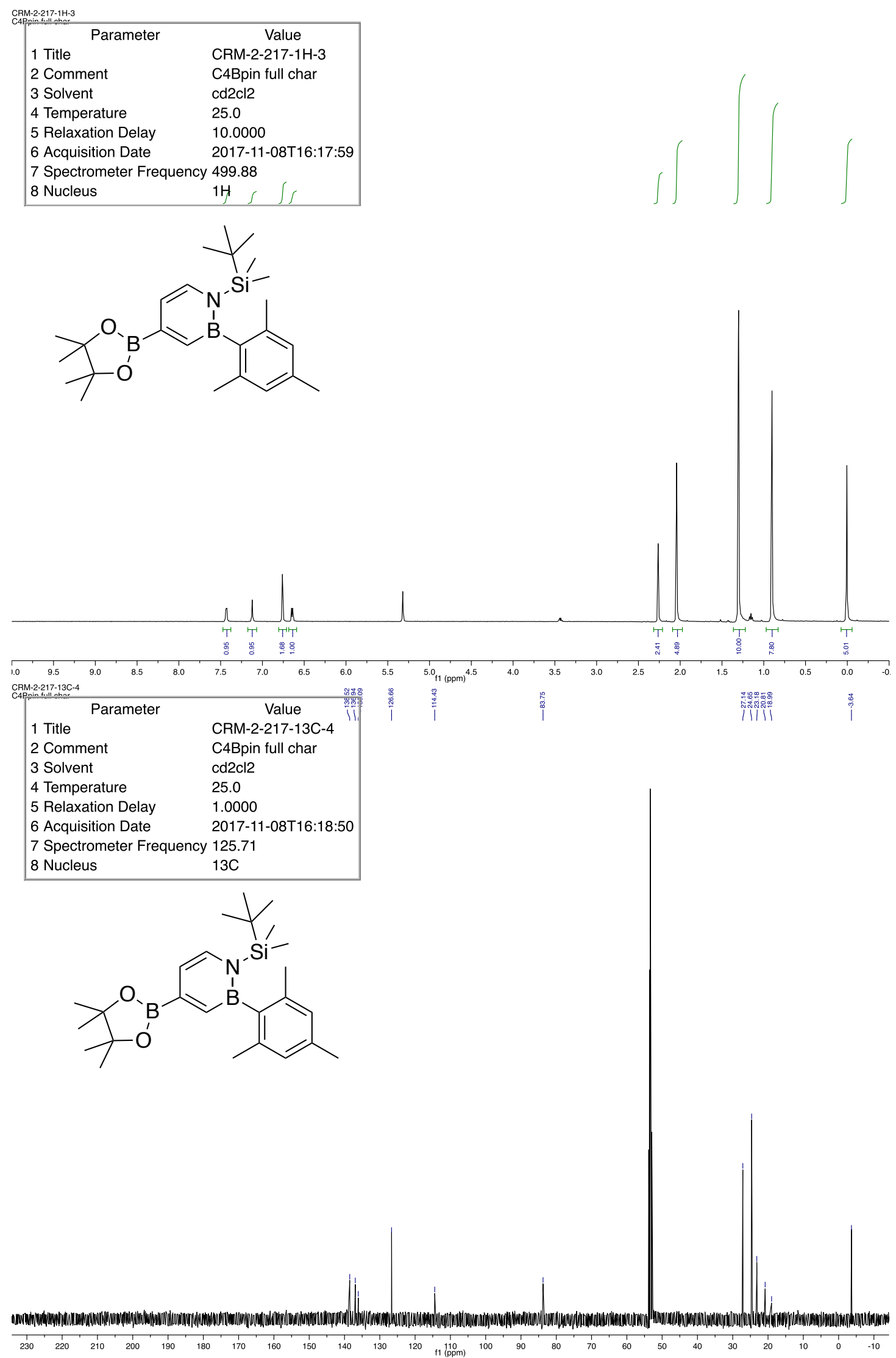




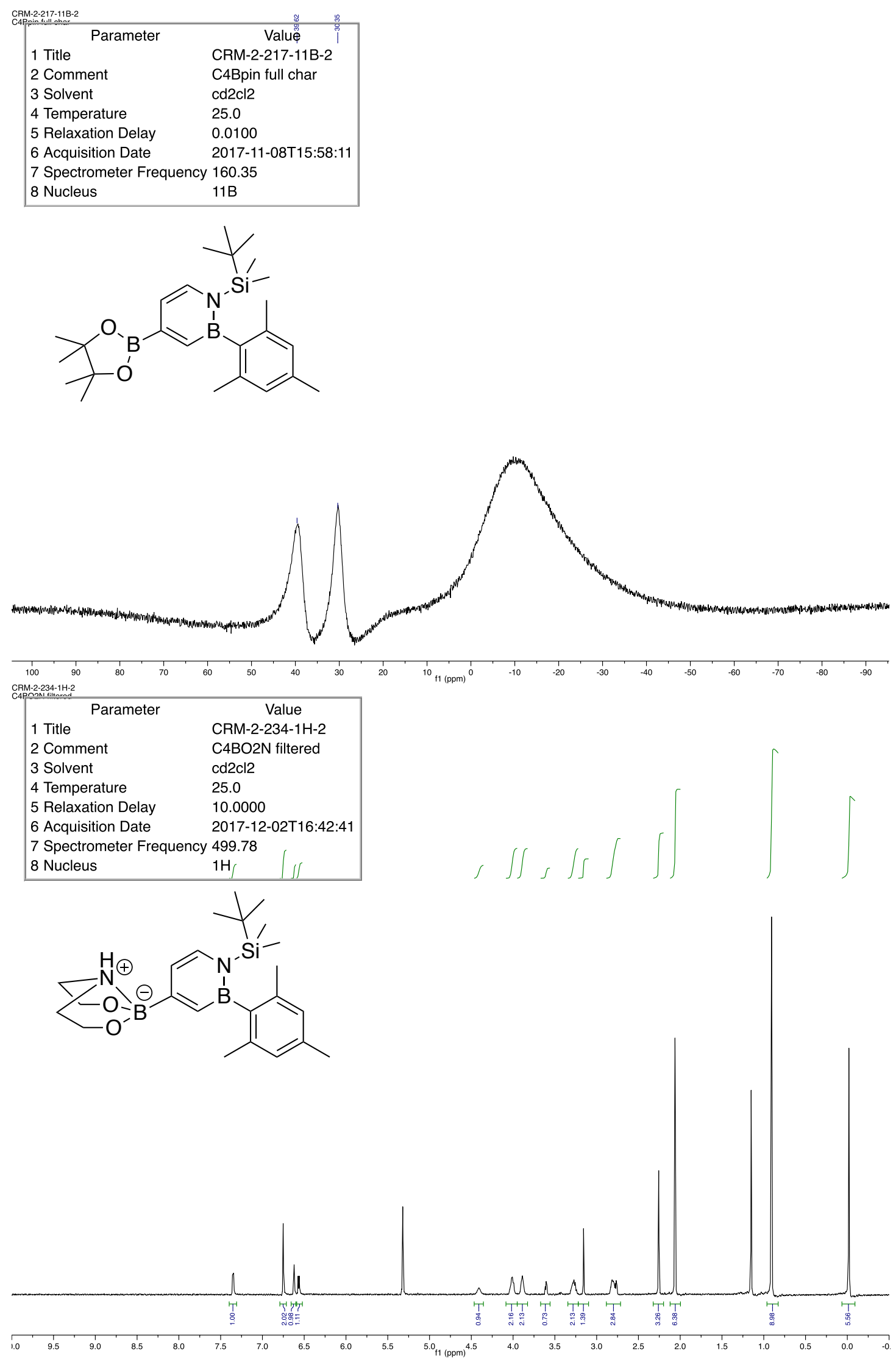




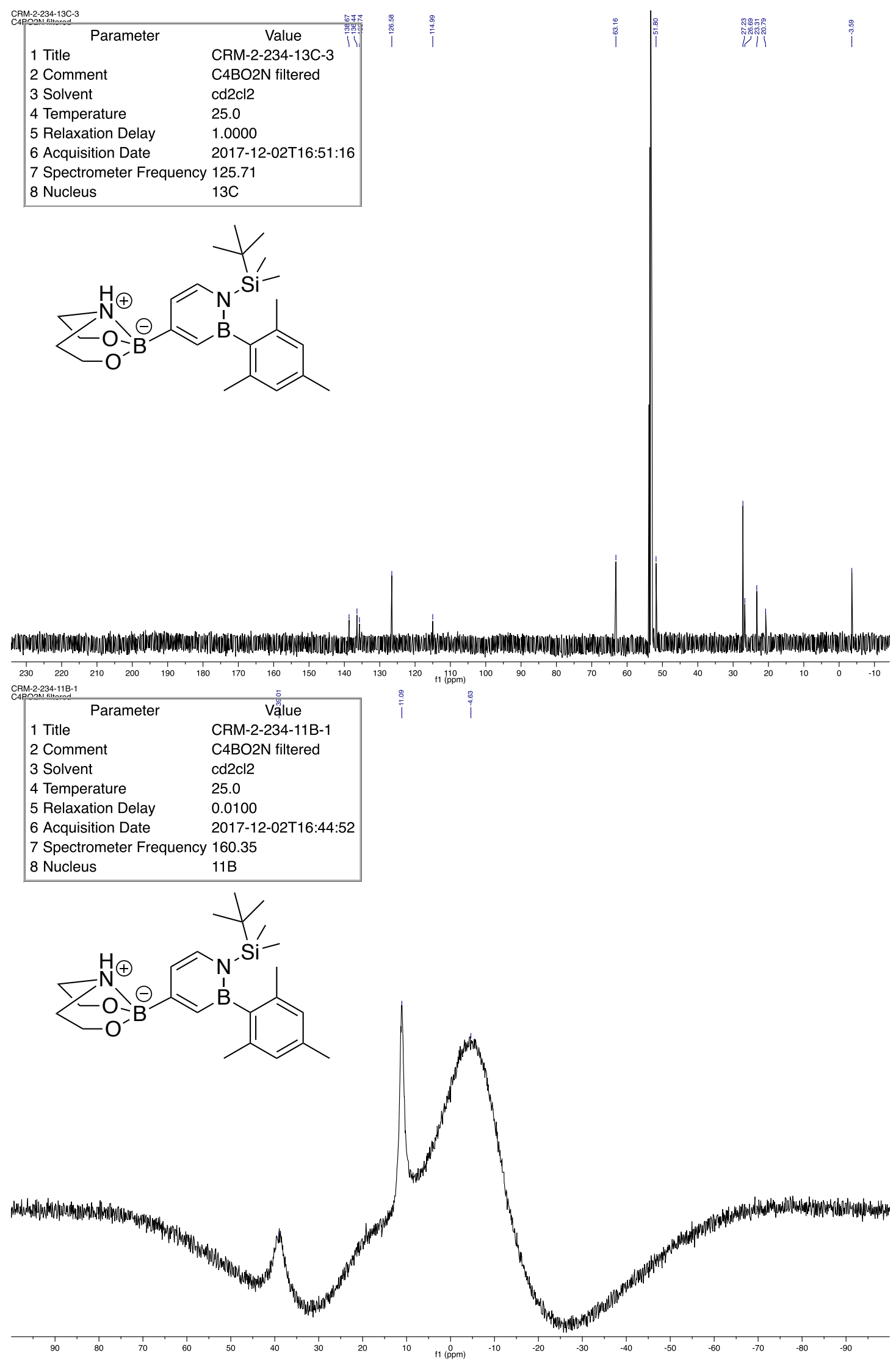




\begin{tabular}{|c|c|}
\hline \multicolumn{2}{|l|}{ 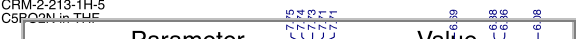 } \\
\hline Parameter & Value \\
\hline 1 Title & CRM-2-213-1H-5 \\
\hline 2 Comment & C5BO2N in THF \\
\hline 3 Solvent & thf \\
\hline 4 Temperature & 25.0 \\
\hline 5 Relaxation Delay & 10.0000 \\
\hline 6 Acquisition Date & 2019-04-01T23:30:40 \\
\hline \multicolumn{2}{|c|}{7 Spectrometer Frequency 499.89} \\
\hline 8 Nucleus & $1 \mathrm{H}$ \\
\hline
\end{tabular}

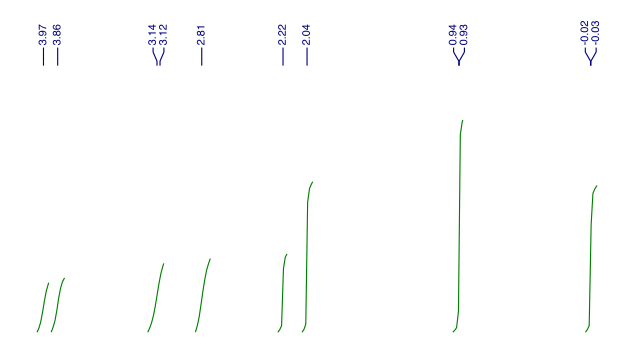<smiles>Cc1cc(C)c(B2C=CC(B3NC4CC(CCO3)C4)=CN2[Si](C)(C)C(C)(C)C)c(C)c1</smiles>

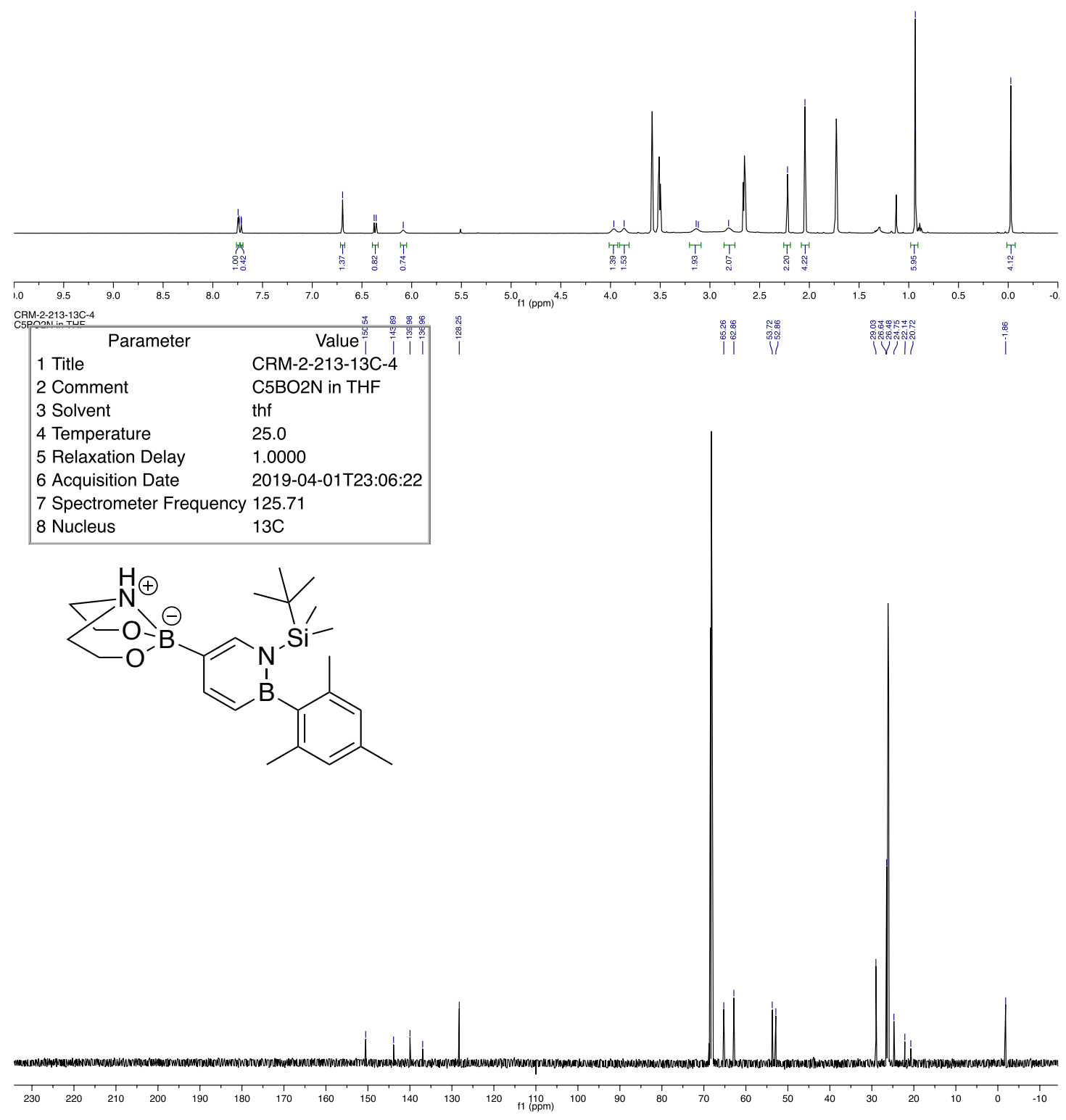




\begin{tabular}{|c|c|}
\hline Parameter & Valuê \\
\hline 1 Title & CRM-2-213-11B-6 \\
\hline 2 Comment & C5BO2N full char thf \\
\hline 3 Solvent & thf \\
\hline 4 Temperature & 25.0 \\
\hline 5 Relaxation Delay & 0.1000 \\
\hline 6 Acquisition Date & 2019-04-02T10:53:57 \\
\hline \multicolumn{2}{|c|}{7 Spectrometer Frequency 160.35} \\
\hline 8 Nucleus & 11B \\
\hline
\end{tabular}<smiles>Cc1cc(C)c(B2C=CC(B3NC4(CCO3)CC4)=CN2[Si](C)(C)C(C)(C)C)c(C)c1</smiles>

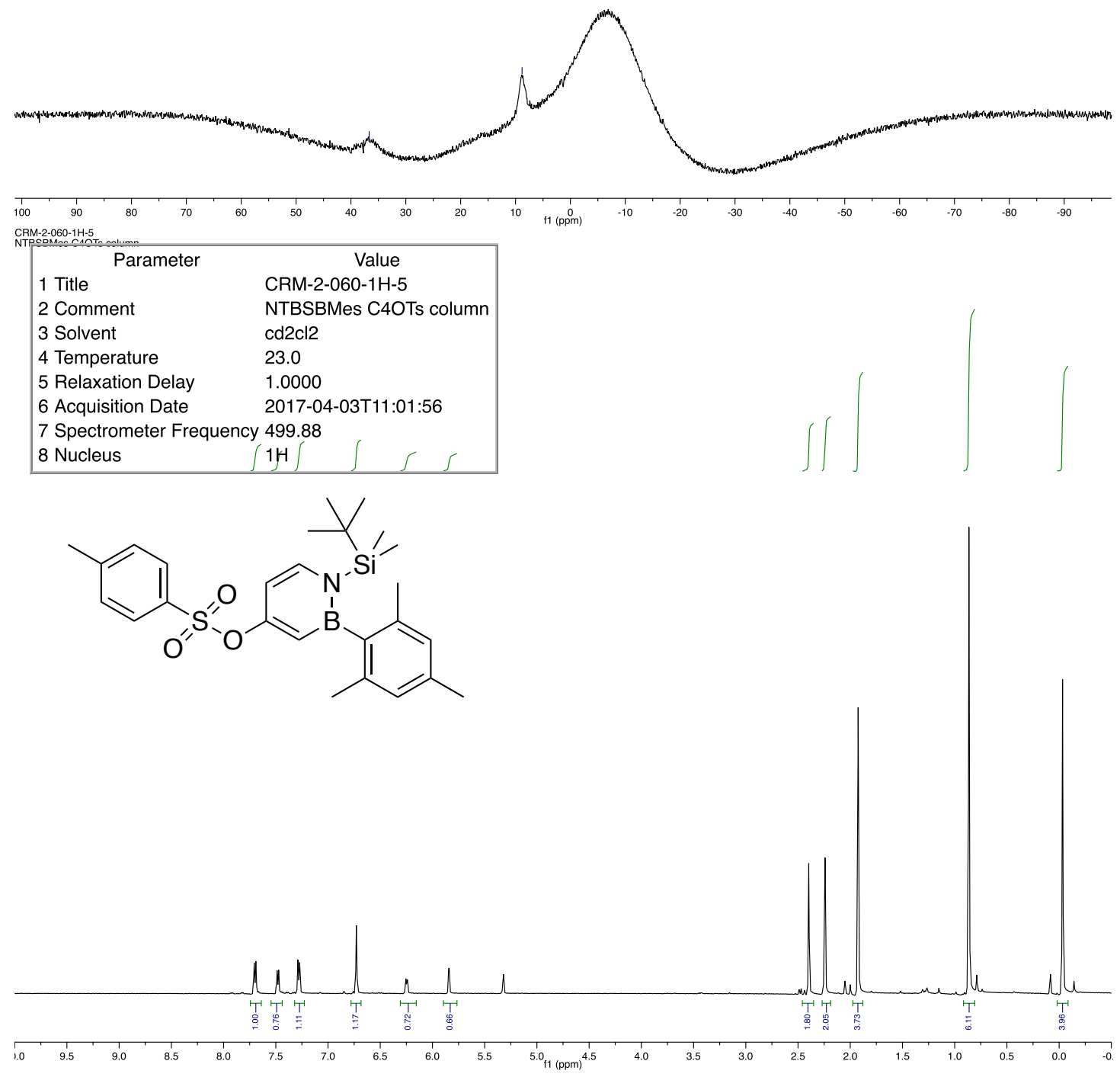



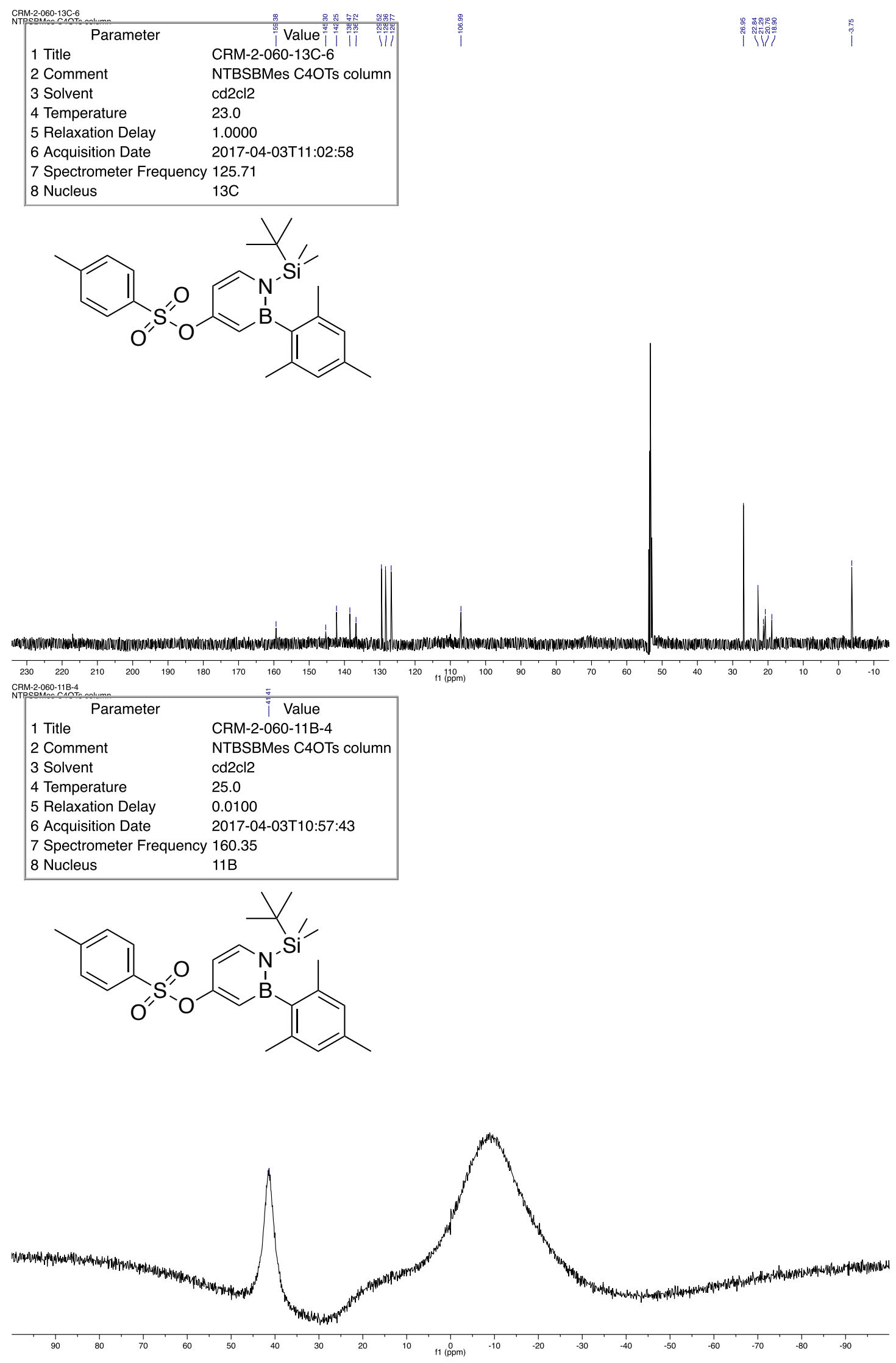

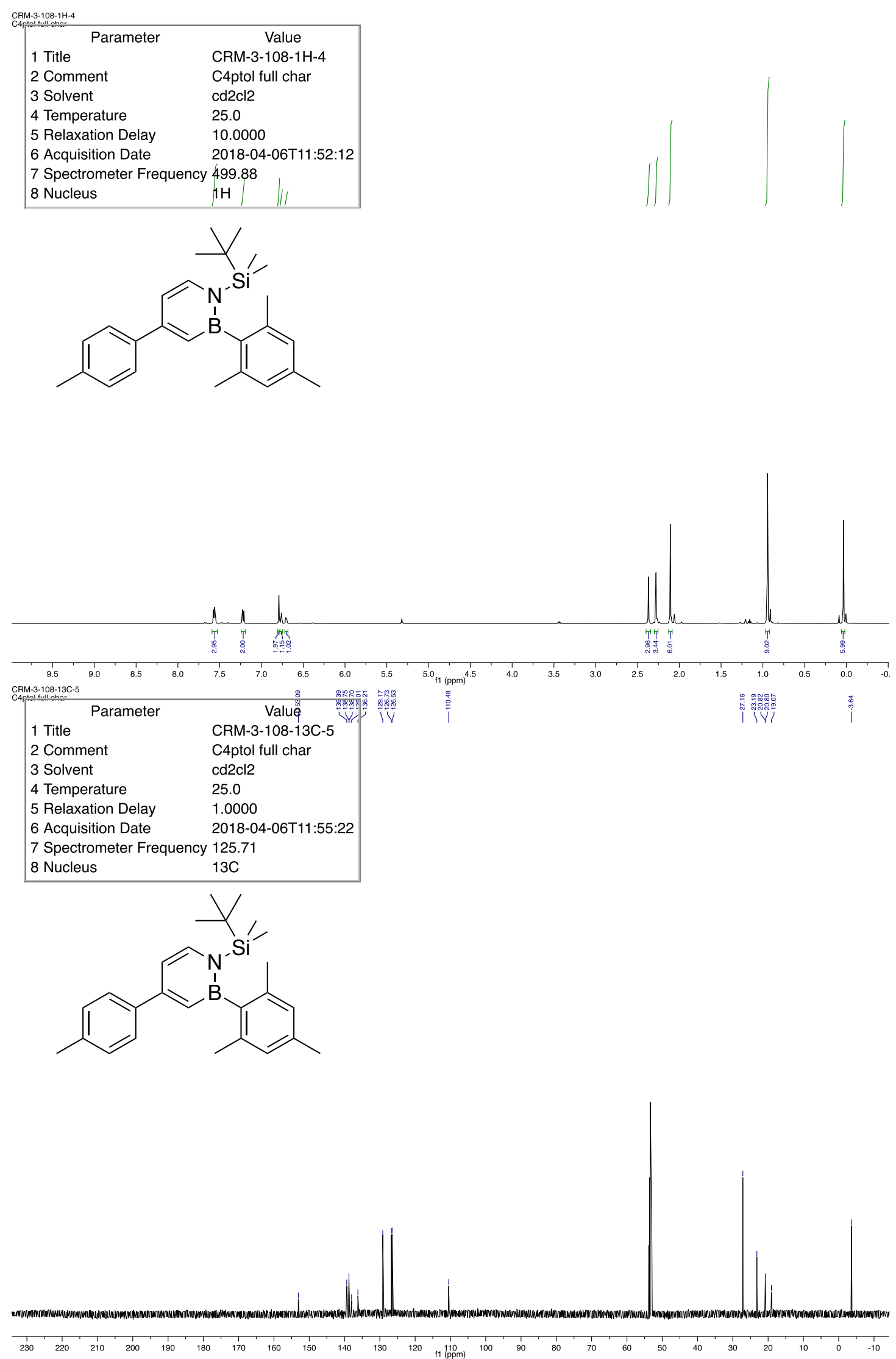

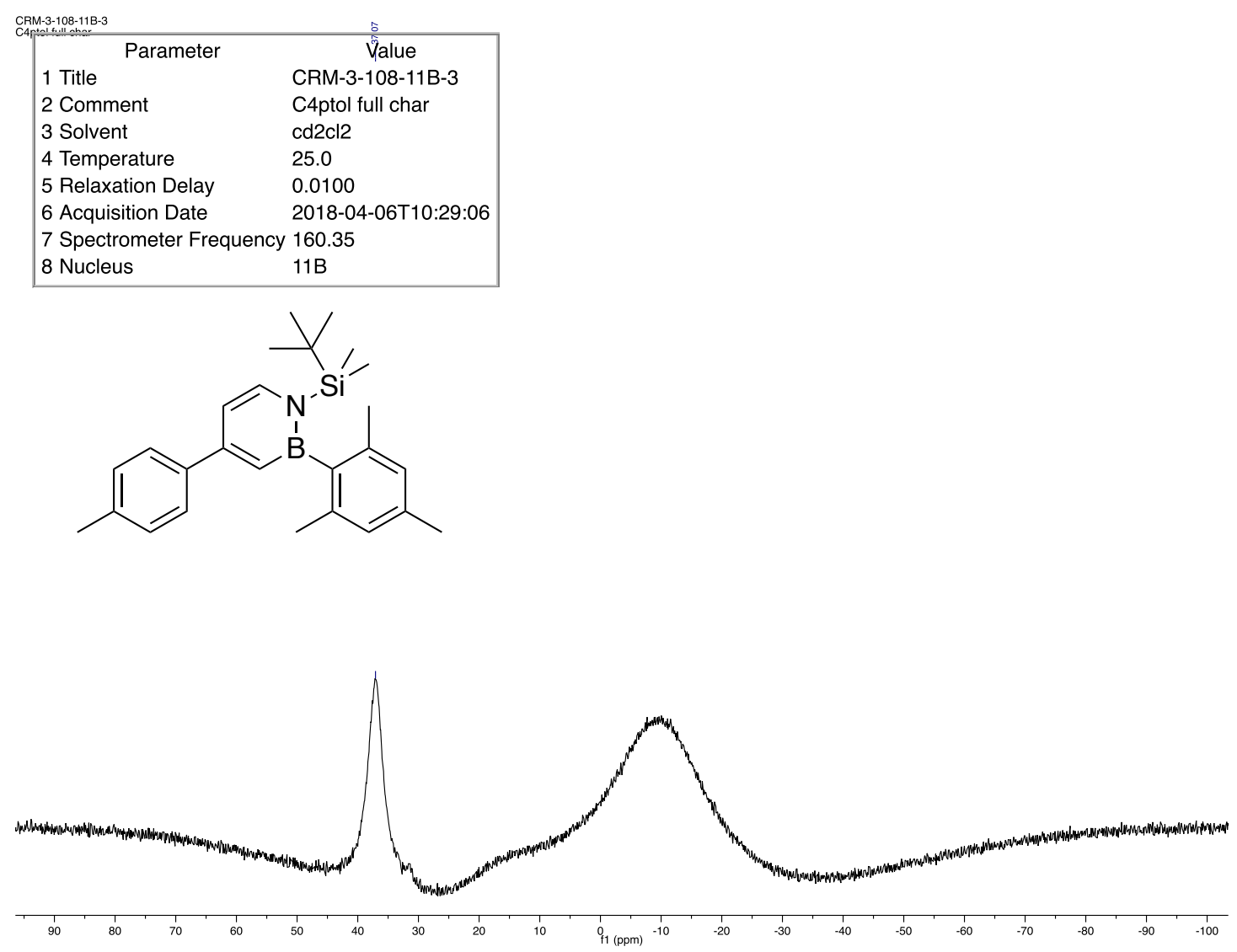

\begin{tabular}{|c|c|}
\hline Parameter & Value \\
\hline 1 Title & C5lodo-fullchar- $1 \mathrm{H}$ \\
\hline \multicolumn{2}{|l|}{2 Comment } \\
\hline 3 Solvent & c6d6 \\
\hline 4 Temperature & 25.0 \\
\hline 5 Relaxation Delay & 10.0000 \\
\hline 6 Acquisition Date & 2018-09-24T19:47:41 \\
\hline \multicolumn{2}{|c|}{7 Spectrometer Frequency 499.88} \\
\hline 8 Nucleus & $\mathrm{r} \mathrm{H}$ \\
\hline
\end{tabular}<smiles>Cc1cc(C)c(B2C=CC(I)=CN2[Si](C)(C)C(C)(C)C)c(C)c1</smiles>

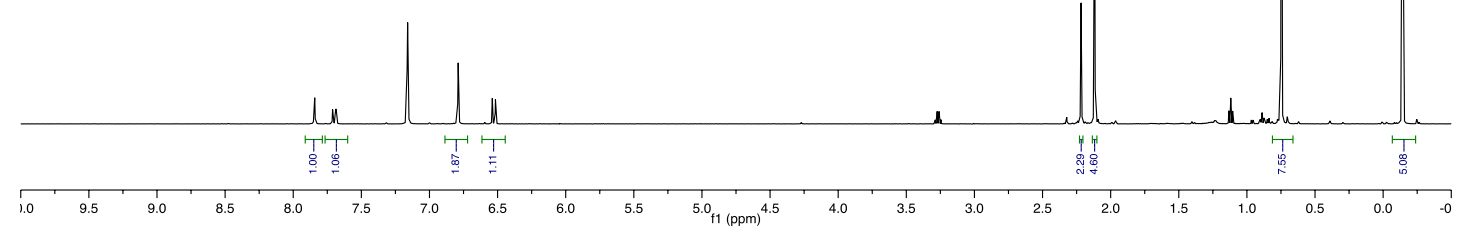



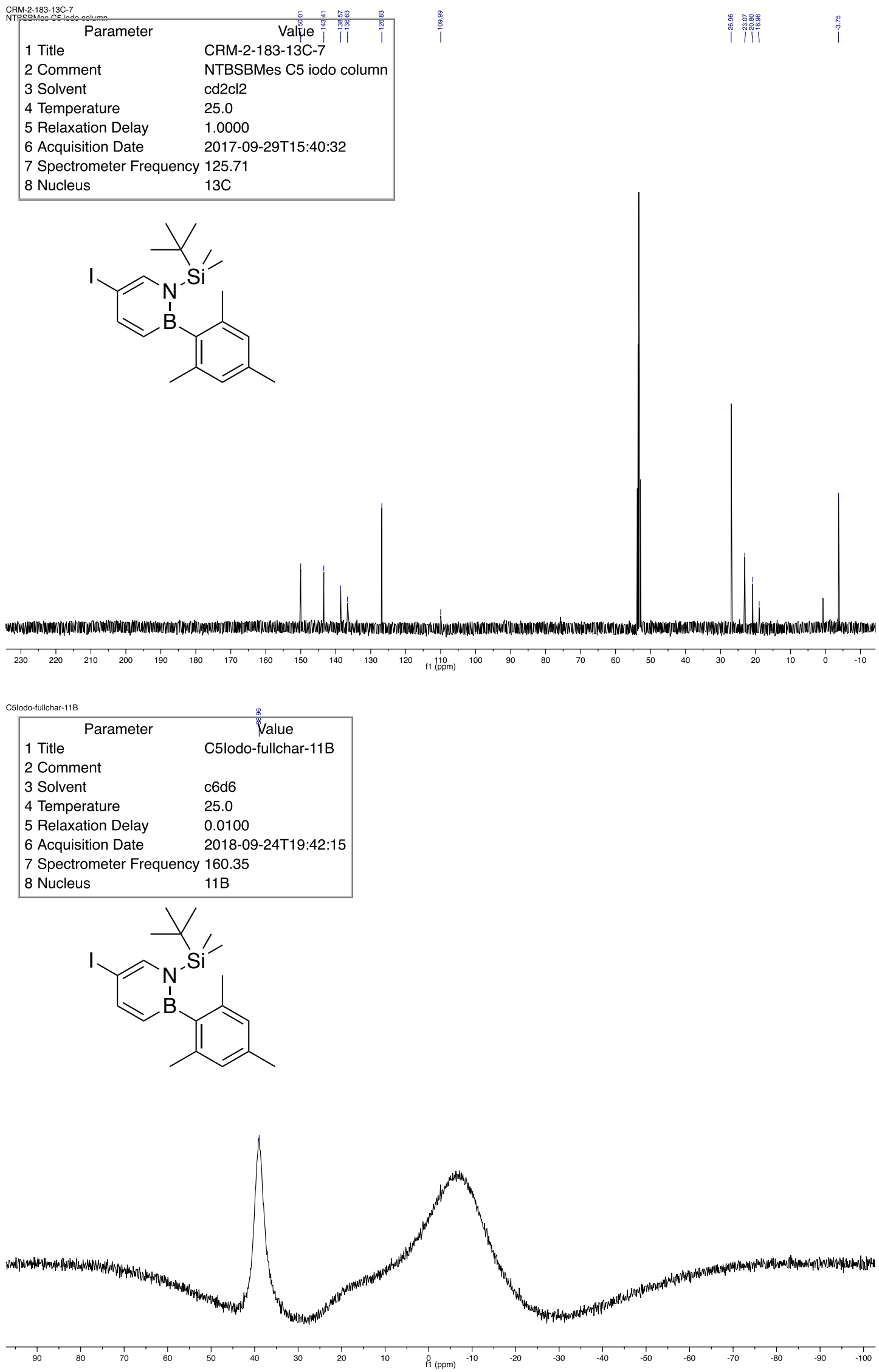

S57 


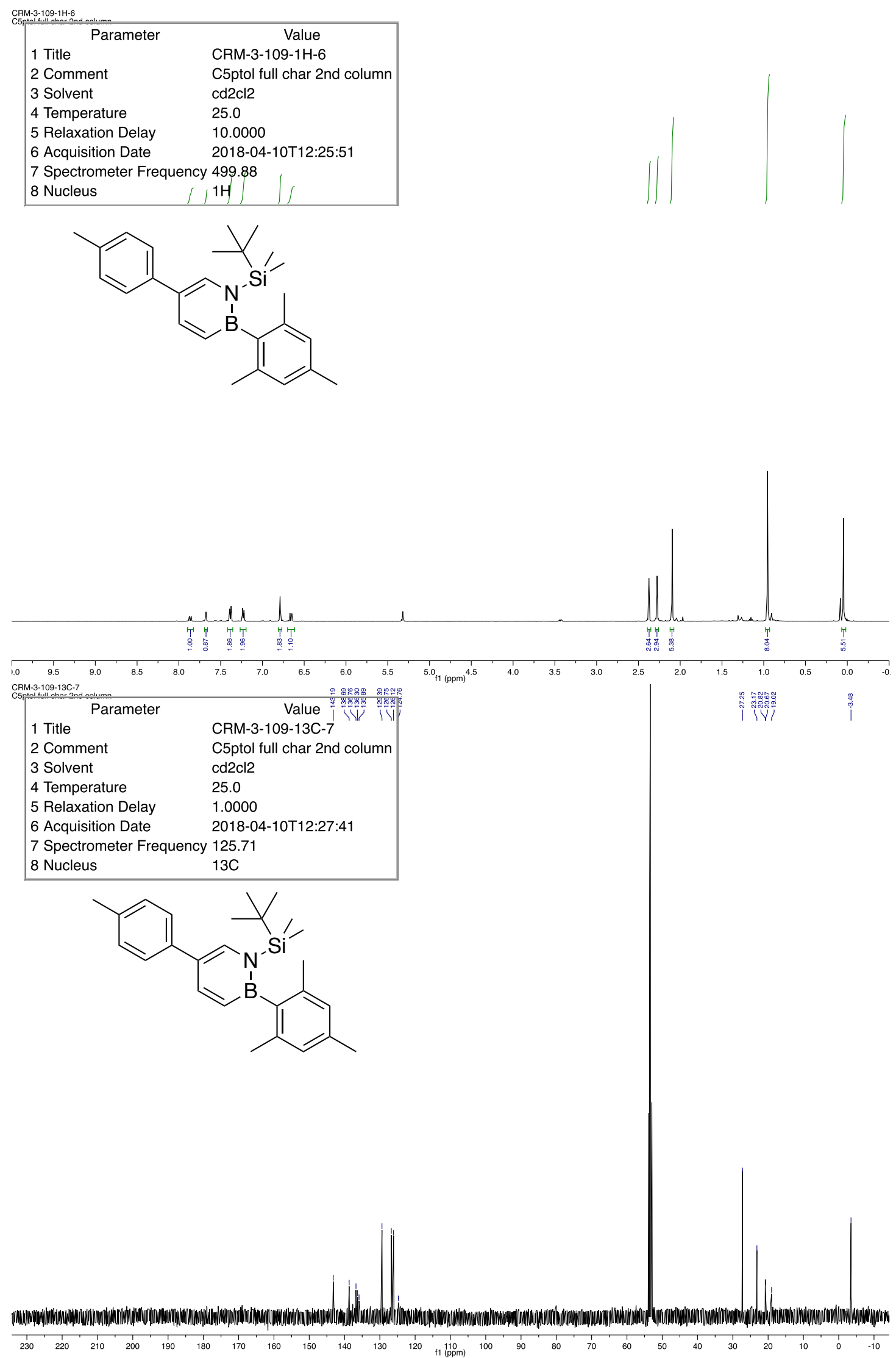




\begin{tabular}{|c|c|}
\hline \multicolumn{2}{|l|}{ 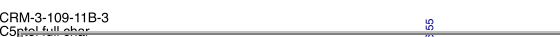 } \\
\hline Parameter & V̆alue \\
\hline 1 Title & CRM-3-109-11B-3 \\
\hline 2 Comment & C5ptol full char \\
\hline 3 Solvent & $\mathrm{cd} 2 \mathrm{cl} 2$ \\
\hline 4 Temperature & 25.0 \\
\hline 5 Relaxation Delay & 0.0100 \\
\hline 6 Acquisition Date & 2018-04-06T10:32:20 \\
\hline \multicolumn{2}{|c|}{7 Spectrometer Frequency 160.35} \\
\hline 8 Nucleus & $11 \mathrm{~B}$ \\
\hline
\end{tabular}<smiles>Cc1ccc(C2=CN([Si](C)(C)C(C)(C)C)B(c3c(C)cc(C)cc3C)C=C2)cc1</smiles>

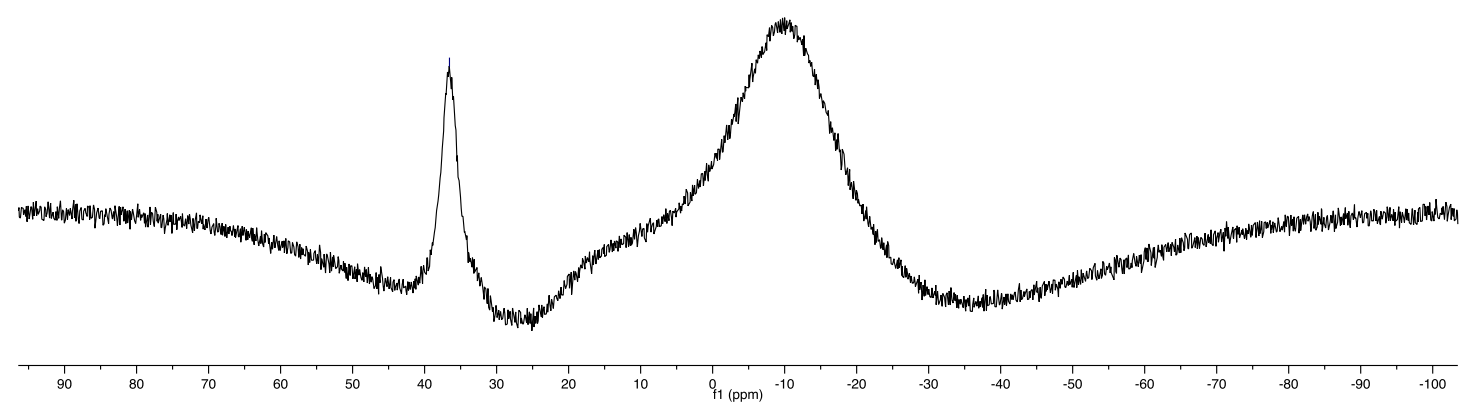

\section{References}

(1) Baggett, A. W.; Vasiliu, M.; Li, B.; Dixon, D. A.; Liu, S.-Y. Late-Stage Functionalization of 1,2-Dihydro-1,2-azaborines via Regioselective IridiumCatalyzed C-H Borylation: The Development of a New N,N-Bidentate Ligand Scaffold. J. Am. Chem. Soc. 2015, 137, 5536-5541.

(2) Frisch, M. J.; Trucks, G. W.; Schlegel, H. B.; Scuseria, G. E.; Robb, M. A.; Cheeseman, J. R.; Scalmani, G.; Barone, V.; Mennucci, B.; Petersson, G. A.; Nakatsuji, H.; Caricato, M.; Li, X.; Hratchian, H. P.; Izmaylov, A. F.; Bloino, J.; Zheng, G.; Sonnenberg, J. L.; Hada, M.; Ehara, M.; Toyota, K.; Fukuda, R.; Hasegawa, J.; Ishida, M.; Nakajima, T.; Honda, Y.; Kitao, O.; Nakai, H.; Vreven, T.; Montgomery, J. A., Jr.; Peralta, J. E.; Ogliaro, F.; Bearpark, M.; Heyd, J. J.; Brothers, E.; Kudin, K. N.; Staroverov, V. N.; Kobayashi, R.; Normand, J.; Raghavachari, K.; Rendell, A.; Burant, J. C.; Iyengar, S. S.; Tomasi, J.; Cossi, M.; Rega, N.; Millam, J. M.; Klene, M.; Knox, J. E.; Cross, J. B.; Bakken, V.; Adamo, C.; Jaramillo, J.; Gomperts, R.; Stratmann, R. E.; Yazyev, O.; Austin, A. J.; Cammi, R.; Pomelli, C.; Ochterski, J. W.; Martin, R. L.; Morokuma, K.; Zakrzewski, V. G.; Voth, G. A.; Salvador, P.; Dannenberg, J. J.; Dapprich, S.; Daniels, A. D.; Farkas, O.; Foresman, J. B.; Ortiz, J. V.; Cioslowski, J.; Fox, D. J. Gaussian 09, Revision C.01; Gaussian Inc., Wallingford, CT, 2010. 